\title{
The Spatially Homogeneous Boltzmann Equation for Bose-Einstein Particles: Rate of Strong Convergence to Equilibrium
}

\author{
Shuzhe Cai* and Xuguang $\mathrm{Lu}^{\dagger}$
}

\begin{abstract}
The paper is a continuation of our previous work on the spatially homogeneous Boltzmann equation for Bose-Einstein particles with quantum collision kernel that includes the hard sphere model. Solutions $F_{t}$ under consideration that conserve the mass, momentum, and energy and converge at least weakly to equilibrium $F_{\text {be }}$ as $t \rightarrow \infty$ have been proven to exist at least for isotropic initial data that have positive entropy, and $F_{t}$ have to be Borel measures for the case of low temperature. The new progress is as follows: we prove that the long time convergence of $F_{t}(\{0\})$ to the Bose-Einstein condensation $F_{\text {be }}(\{0\})$ holds for all isotropic initial data $F_{0}$ satisfying the low temperature condition. This immediately implies the long time strong convergence to equilibrium. We also obtain an algebraic rate of the strong convergence for arbitrary temperature. Our proofs are based on entropy control, positive lower bound of entropy, Villani's inequality for entropy dissipation, a suitable time-dependent convex combination between the solution and a fixed positive function (in order to deal with logarithm terms), the convex-positivity of the cubic collision integral, and an iteration technique for obtaining a positive lower bound of condensation.
\end{abstract}

Key words: Bose-Einstein particles, entropy, strong convergence, equilibrium, low temperature, condensation.

\section{Introduction}

The quantum Boltzmann equations for Bose-Einstein particles and for Fermi-Dirac particles (which are also called Boltzmann-Nordheim equation, Uehling-Uhlenbeck equation, etc.) were first derived by Nordheim [26] and Uehling \& Uhlenbeck [32] and then taken attention and developed by [4],[7],[9],[24]. For the case of Bose-Einstein particles and for the spatially homogeneous solutions, the equation under consideration is written

$$
\frac{\partial}{\partial t} f(\mathbf{v}, t)=\int_{\mathbb{R}^{3} \times \mathbb{S}^{2}} B\left(\mathbf{v}-\mathbf{v}_{*}, \omega\right)\left(f^{\prime} f_{*}^{\prime}(1+f)\left(1+f_{*}\right)-f f_{*}\left(1+f^{\prime}\right)\left(1+f_{*}^{\prime}\right)\right) \mathrm{d} \omega \mathrm{d} \mathbf{v}_{*}
$$

with $(\mathbf{v}, t) \in \mathbb{R}^{3} \times(0, \infty)$, where the solution $f=f(\mathbf{v}, t) \geq 0$ is the number density of particles at time $t$ with the velocity $\mathbf{v}$, and as usual we denote briefly $f_{*}=f\left(\mathbf{v}_{*}, t\right), f^{\prime}=f\left(\mathbf{v}^{\prime}, t\right), f_{*}^{\prime}=$

*Department of Mathematical Sciences, Tsinghua University, Beijing 100084, P.R.China; e-mail address: csz16@mails.tsinghua.edu.cn

${ }^{\dagger}$ Department of Mathematical Sciences, Tsinghua University, Beijing 100084, P.R.China; e-mail address: xglu@math.tsinghua.edu.cn 
$f\left(\mathbf{v}_{*}^{\prime}, t\right)$ where $\mathbf{v}, \mathbf{v}_{*}$ and $\mathbf{v}^{\prime}, \mathbf{v}_{*}^{\prime}$ are velocities of two particles before and after their collision:

$$
\mathbf{v}^{\prime}=\mathbf{v}-\left(\left(\mathbf{v}-\mathbf{v}_{*}\right) \cdot \omega\right) \omega, \quad \mathbf{v}_{*}^{\prime}=\mathbf{v}_{*}+\left(\left(\mathbf{v}-\mathbf{v}_{*}\right) \cdot \omega\right) \omega, \quad \omega \in \mathbb{S}^{2}
$$

which conserves the momentum and kinetic energy

$$
\mathbf{v}^{\prime}+\mathbf{v}_{*}^{\prime}=\mathbf{v}+\mathbf{v}_{*}, \quad\left|\mathbf{v}^{\prime}\right|^{2}+\left|\mathbf{v}_{*}^{\prime}\right|^{2}=|\mathbf{v}|^{2}+\left|\mathbf{v}_{*}\right|^{2} .
$$

The function $B\left(\mathbf{v}-\mathbf{v}_{*}, \omega\right)$ is the collision kernel and is assumed to take the following general form in order to include possible models:

$$
B\left(\mathbf{v}-\mathbf{v}_{*}, \omega\right)=\frac{1}{(4 \pi)^{2}}\left|\left(\mathbf{v}-\mathbf{v}_{*}\right) \cdot \omega\right| \Phi\left(\left|\mathbf{v}-\mathbf{v}^{\prime}\right|,\left|\mathbf{v}-\mathbf{v}_{*}^{\prime}\right|\right)
$$

where

$$
0 \leq \Phi \in C_{b}\left(\mathbb{R}_{\geq 0}^{2}\right), \quad \Phi(r, \rho)=\Phi(\rho, r) \quad \forall(r, \rho) \in \mathbb{R}_{\geq 0}^{2} .
$$

According to [4] and [9] in the weak-coupling regime, the function $\Phi$ takes the following form (after normalizing physical parameters)

$$
\Phi(r, \rho)=(\widehat{\phi}(r)+\widehat{\phi}(\rho))^{2}, \quad r, \rho \geq 0
$$

where $\widehat{\phi}$ is the Fourier transform (in terms of theory of generalized functions ) of a radially symmetric particle interaction potential $\phi(|\mathbf{x}|) \in \mathbb{R}$ :

$$
\widehat{\phi}(r):=\left.\widehat{\phi}(\xi)\right|_{|\xi|=r}=\left.\int_{\mathbb{R}^{3}} \phi(|\mathbf{x}|) e^{-\mathrm{i} \xi \cdot \mathbf{x}} \mathrm{d} \mathbf{x}\right|_{|\xi|=r} .
$$

In particular if $\phi(|\mathbf{x}|)=\frac{1}{2} \delta(\mathbf{x})$, where $\delta(\mathbf{x})$ is the three dimensional Dirac delta function concentrating at $\mathbf{x}=0$, then $\widehat{\phi} \equiv \frac{1}{2}$ hence $\Phi \equiv 1$ and (1.4) becomes the hard sphere model:

$$
B\left(\mathbf{v}-\mathbf{v}_{*}, \omega\right)=\frac{1}{(4 \pi)^{2}}\left|\left(\mathbf{v}-\mathbf{v}_{*}\right) \cdot \omega\right|
$$

which is the only model that has the same form as in the classical Boltzmann equation, and has been mainly concerned in many papers about Eq.(1.1). In view of physics, the hard sphere model (1.7) can be extended by allowing the interaction potential $\phi(\mathbf{x})$ contains an attractive long-range term $\frac{-1}{2} U(|\mathbf{x}|)$, i.e. $\phi(|\mathbf{x}|)=\frac{1}{2}(\delta(\mathbf{x})-U(|\mathbf{x}|))$ where $U(|\mathbf{x}|) \geq 0$ and, for a technical reason in proving the occurrence of and convergence to the condensation, we consider such a case that the Fourier transform of $\widehat{U}(\xi)$ of $U(|\mathbf{x}|)$ behaves as $\left.\widehat{U}(\xi)\right|_{|\xi|=r}=\frac{1}{1+r^{\eta}}$ with $0<\eta<1$ so that

$$
\widehat{\phi}(r)=\frac{1}{2}\left(1-\left.\widehat{U}(\xi)\right|_{|\xi|=r}\right)=\frac{1}{2} \cdot \frac{r^{\eta}}{1+r^{\eta}}, \quad r \geq 0
$$

see Appendix for the existence and positivity of such potentials $U$.

In order to include the hard sphere model and the case (1.6) with (1.8) we introduce the following assumption: 
Assumption 1.1. $B\left(\mathbf{v}-\mathbf{v}_{*}, \omega\right)$ is given by (1.4),(1.5) where $\Phi$ also satisfies

$$
\begin{aligned}
& r \mapsto \Phi(r, \rho) \text { is non - decreasing on } \mathbb{R}_{\geq 0} \quad \forall \rho \in \mathbb{R}_{\geq 0}, \\
& b_{0} \min \left\{1,\left(r^{2}+\rho^{2}\right)^{\eta}\right\} \leq \Phi(r, \rho) \leq 1 \quad \forall(r, \rho) \in \mathbb{R}_{\geq 0}^{2}
\end{aligned}
$$

for some constants $0<b_{0}<1, \eta \geq 0$.

The monotone assumption (1.9) together with a further restriction $0 \leq \eta<1 / 4$ is mainly used to prove the convex positivity of the cubic collision integral (see Proposition 5.1) and the convergence of condensation $F_{t}(\{0\}) \rightarrow F_{\text {be }}(\{0\})(t \rightarrow \infty)$. Except these, Assumption 1.1 says that the collision kernel $B\left(\mathbf{v}-\mathbf{v}_{*}, \omega\right)$ maybe vanishes at $\mathbf{v}-\mathbf{v}_{*}=0$ but still behaves like the hard sphere model for large $\left|\mathbf{v}-\mathbf{v}_{*}\right|$ : for all $\mathbf{v}, \mathbf{v}_{*} \in \mathbb{R}^{3}, \omega \in \mathbb{S}^{2}$

$$
\frac{b_{0}}{(4 \pi)^{2}}\left|\left(\mathbf{v}-\mathbf{v}_{*}\right) \cdot \omega\right| \leq B\left(\mathbf{v}-\mathbf{v}_{*}, \omega\right) \leq \frac{1}{(4 \pi)^{2}}\left|\left(\mathbf{v}-\mathbf{v}_{*}\right) \cdot \omega\right| \quad \text { with }\left|v-v_{*}\right| \geq 1
$$

Due to the strong nonlinear structure of the collision integrals and the effect of condensation, existence and uniqueness of solutions of Eq.(1.1) for anisotropic initial data have been so far only proven for finite time interval without smallness assumption on the initial data [5] and for global time interval with a smallness assumption on the initial data [17]. For global in time solutions with general initial data, in particular for the case of low temperature, one has to consider weak solutions $f$ which are solutions of the following equation

$$
\begin{aligned}
\frac{\mathrm{d}}{\mathrm{d} t} \int_{\mathbb{R}^{3}} \psi(\mathbf{v}) f(\mathbf{v}, t) \mathrm{d} \mathbf{v} & =\frac{1}{2} \int_{\mathbb{R}^{3} \times \mathbb{R}^{3} \times \mathbb{S}^{2}}\left(\psi+\psi_{*}-\psi^{\prime}-\psi_{*}^{\prime}\right) B\left(\mathbf{v}-\mathbf{v}_{*}, \omega\right) f^{\prime} f_{*}^{\prime} \mathrm{d} \mathbf{v} \mathrm{d} \mathbf{v}_{*} \mathrm{~d} \omega \\
& +\int_{\mathbb{R}^{3} \times \mathbb{R}^{3} \times \mathbb{S}^{2}}\left(\psi+\psi_{*}-\psi^{\prime}-\psi_{*}^{\prime}\right) B\left(\mathbf{v}-\mathbf{v}_{*}, \omega\right) f f^{\prime} f_{*}^{\prime} \mathrm{d} \mathbf{v} \mathrm{d} \mathbf{v}_{*} \mathrm{~d} \omega
\end{aligned}
$$

for all test functions $\psi$ and all $t \in[0, \infty)$. Here we have used the fact that the common quartic terms $f^{\prime} f_{*}^{\prime} f f_{*}, f f_{*} f^{\prime} f_{*}^{\prime}$ cancel each other:

$$
f^{\prime} f_{*}^{\prime}(1+f)\left(1+f_{*}\right)-f f_{*}\left(1+f^{\prime}\right)\left(1+f_{*}^{\prime}\right)=f^{\prime} f_{*}^{\prime}\left(1+f+f_{*}\right)-f f_{*}\left(1+f^{\prime}+f_{*}^{\prime}\right) .
$$

In general however the cubic integral is divergent:

$$
\sup _{f} \int_{\mathbb{R}^{3} \times \mathbb{R}^{3} \times \mathbb{S}^{2}} \Psi\left(\mathbf{v}, \mathbf{v}^{\prime}, \mathbf{v}_{*}^{\prime}\right) B\left(\mathbf{v}-\mathbf{v}_{*}, \omega\right) f(\mathbf{v}) f\left(\mathbf{v}^{\prime}\right) f\left(\mathbf{v}_{*}^{\prime}\right) \mathrm{d} \mathbf{v} \mathrm{d} \mathbf{v}_{*} \mathrm{~d} \omega=\infty
$$

where $\Psi \in C_{b}\left(\mathbb{R}^{9}\right)$ is an arbitrary positive function and $f$ under the sup is taken all nonnegative functions in $C_{c}^{\infty}\left(\mathbb{R}^{3}\right)$ satisfying $\int_{\mathbb{R}^{3}} f(\mathbf{v}) \mathrm{d} \mathbf{v} \leq 1$ (see e.g. [19]). A subclass of $f$ that has no such divergence is the isotropic (i.e. radially symmetric) functions: $f(\mathbf{v})=f\left(|\mathbf{v}|^{2} / 2\right)$. By changing variables $x=|\mathbf{v}|^{2} / 2, y=\left|\mathbf{v}^{\prime}\right|^{2} / 2, z=\left|\mathbf{v}_{*}^{\prime}\right|^{2} / 2$, one has

$$
B\left(\mathbf{v}-\mathbf{v}_{*}, \omega\right) f\left(|\mathbf{v}|^{2} / 2\right) f\left(\left|\mathbf{v}^{\prime}\right|^{2} / 2\right) f\left(\left|\mathbf{v}_{*}^{\prime}\right|^{2} / 2\right) \mathrm{d} \mathbf{v} \mathrm{d} \mathbf{v}_{*} \mathrm{~d} \omega=4 \pi \sqrt{2} W(x, y, z) \mathrm{d} F(x) \mathrm{d} F(y) \mathrm{d} F(z)
$$


where $\mathrm{d} F(x)=f(x) \sqrt{x} \mathrm{~d} x$, etc., and $x, y, z \in \mathbb{R}_{\geq 0}$ in the right side are independent variables, see below for details. This is the main reason that all results obtained so far (except the ones mentioned above) are concerned with isotropic initial data hence isotropic solutions, see e.g.[11][16],[18]-[23],[27],[29],[30],[31]. Despite this shortage, results obtained so far have shown that the Eq.(1.1) can be used to describe the formation, transition, and propagation of the Bose-Einstein condensation of dilute Bose gases at low temperature, see Theorem 1.4 and Proposition 5.6 below, see also (for instance) [16],[25],[29],[30],[31] for self-similar structure and deterministic numerical methods; [3],[12],[13],[14],[21], for singular solutions and the formation of blow-up and condensation in finite time; [20], [22], [23] for long time strong and weak convergence to the Bose-Einstein distribution; [15] for a linearized model of Eq.(1.1) and rate of convergence to equilibrium; and [1],[2],[27] for general discussions and basic results for similar models on low temperature evolution of condensation.

Before stating the main result of the paper we introduce some notations and the definition of measure-valued isotropic solution of Eq.(1.1). Let $L_{s}^{1}\left(\mathbb{R}^{3}\right)$ with $s \geq 0$ be the linear space of the weighted Lebesgue integrable functions defined by $L_{0}^{1}\left(\mathbb{R}^{3}\right)=L^{1}\left(\mathbb{R}^{3}\right)$ and

$$
L_{s}^{1}\left(\mathbb{R}^{3}\right)=\left\{f \in L^{1}\left(\mathbb{R}^{3}\right)\left|\|f\|_{L_{s}^{1}}:=\int_{\mathbb{R}^{3}}\langle\mathbf{v}\rangle^{s}\right| f(\mathbf{v}) \mid \mathrm{d} \mathbf{v}<\infty\right\}, \quad\langle\mathbf{v}\rangle:=\left(1+|\mathbf{v}|^{2}\right)^{1 / 2} .
$$

Let $\mathcal{B}_{k}(X)(k \geq 0)$ be the linear space of signed real Borel measures $F$ on a Borel set $X \subset \mathbb{R}^{d}$ satisfying $\int_{X}(1+|x|)^{k} \mathrm{~d}|F|(x)<\infty$, where $|F|$ is the total variation of $F$. Let

$$
\mathcal{B}_{k}^{+}(X)=\left\{F \in \mathcal{B}_{k}(X) \mid F \geq 0\right\}
$$

For the case $k=0$ we also denote $\mathcal{B}(X)=\mathcal{B}_{0}(X), \mathcal{B}^{+}(X)=\mathcal{B}_{0}^{+}(X)$. In this paper we only consider two cases $X=\mathbb{R}^{3}$ and $X=\mathbb{R}_{\geq 0}$, and in many cases we consider isotropic measures $\bar{F} \in \mathcal{B}_{2 k}\left(\mathbb{R}^{3}\right)$, which define and can be defined by measures $F \in \mathcal{B}_{k}\left(\mathbb{R}_{\geq 0}\right)$ in terms of the following relations:

$$
\begin{gathered}
F(A)=\frac{1}{4 \pi \sqrt{2}} \int_{\mathbb{R}^{3}} \mathbf{1}_{A}\left(|\mathbf{v}|^{2} / 2\right) \mathrm{d} \bar{F}(\mathbf{v}), \quad A \subset \mathbb{R}_{\geq 0} \\
\bar{F}(B)=4 \pi \sqrt{2} \int_{\mathbb{R}_{\geq 0}}\left(\frac{1}{4 \pi} \int_{\mathbb{S}^{2}} \mathbf{1}_{B}(\sqrt{2 x} \omega) \mathrm{d} \omega\right) \mathrm{d} F(x), \quad B \subset \mathbb{R}^{3}
\end{gathered}
$$

for all Borel measurable sets $A, B$. They are equivalent to functional forms:

$$
\begin{gathered}
\int_{\mathbb{R}_{\geq 0}} \varphi(x) \mathrm{d} F(x)=\frac{1}{4 \pi \sqrt{2}} \int_{\mathbb{R}^{3}} \varphi\left(|\mathbf{v}|^{2} / 2\right) \mathrm{d} \bar{F}(\mathbf{v}), \\
\int_{\mathbb{R}^{3}} \psi(\mathbf{v}) \mathrm{d} \bar{F}(\mathbf{v})=4 \pi \sqrt{2} \int_{\mathbb{R}_{\geq 0}}\left(\frac{1}{4 \pi} \int_{\mathbb{S}^{2}} \psi(\sqrt{2 x} \omega) \mathrm{d} \omega\right) \mathrm{d} F(x)
\end{gathered}
$$

for all bounded Borel measurable functions $\varphi, \psi$. For any $k \geq 0$ let

$$
\|F\|_{k}=\int_{\mathbb{R}_{\geq 0}}(1+x)^{k} \mathrm{~d}|F|(x), \quad F \in \mathcal{B}_{k}\left(\mathbb{R}_{\geq 0}\right) .
$$


The moment $M_{p}(F)$ of order $p \in[0, k]$ of $F \in \mathcal{B}_{k}^{+}\left(\mathbb{R}_{\geq 0}\right)$ is defined by

$$
M_{p}(F)=\int_{\mathbb{R}_{\geq 0}} x^{p} \mathrm{~d} F(x) .
$$

Moments of orders 0,1 correspond to the mass and energy and are particularly denoted as $N(F)=M_{0}(F), E(F)=M_{1}(F)$, i.e.

$$
N(F)=\int_{\mathbb{R}_{\geq 0}} \mathrm{~d} F(x), \quad E(F)=\int_{\mathbb{R}_{\geq 0}} x \mathrm{~d} F(x) .
$$

Let $C_{b}^{k}\left(\mathbb{R}_{\geq 0}\right)$ with $k \in \mathbb{N}$ be the class of bounded continuous functions on $\mathbb{R}_{\geq 0}$ having bounded continuous derivatives on $\mathbb{R}_{\geq 0}$ up to the order $k$. For isotropic functions $f=f\left(|\mathbf{v}|^{2} / 2\right) \geq 0$, $\varphi=\varphi\left(|\mathbf{v}|^{2} / 2\right)$ with $f\left(|\cdot|^{2} / 2\right) \in L_{2}^{1}\left(\mathbb{R}^{3}\right), \varphi \in C_{b}^{2}\left(\mathbb{R}_{\geq 0}\right)$, and for the measure $F$ defined by $\mathrm{d} F(x)=f(x) \sqrt{x} \mathrm{~d} x$, the collision integrals in (1.12) can be rewritten (see Appendix)

$$
\begin{aligned}
& \frac{1}{2} \int_{\mathbb{R}^{3} \times \mathbb{R}^{3} \times \mathbb{S}^{2}}\left(\varphi+\varphi_{*}-\varphi^{\prime}-\varphi_{*}^{\prime}\right) B f^{\prime} f_{*}^{\prime} \mathrm{d} \mathbf{v} \mathrm{d} \mathbf{v}_{*} \mathrm{~d} \omega=4 \pi \sqrt{2} \int_{\mathbb{R}_{\geq 0}^{2}} \mathcal{J}[\varphi] \mathrm{d}^{2} F \\
& \int_{\mathbb{R}^{3} \times \mathbb{R}^{3} \times \mathbb{S}^{2}}\left(\varphi+\varphi_{*}-\varphi^{\prime}-\varphi_{*}^{\prime}\right) B f f^{\prime} f_{*}^{\prime} \mathrm{d} \mathbf{v} \mathrm{d} \mathbf{v}_{*} \mathrm{~d} \omega=4 \pi \sqrt{2} \int_{\mathbb{R}_{\geq 0}^{3}} \mathcal{K}[\varphi] \mathrm{d}^{3} F
\end{aligned}
$$

where $B=B\left(\mathbf{v}-\mathbf{v}_{*}, \omega\right)$ is given by (1.4) with (1.5), $\mathrm{d}^{2} F=\mathrm{d} F(y) \mathrm{d} F(z), \mathrm{d}^{3} F=\mathrm{d} F(x) \mathrm{d} F(y) \mathrm{d} F(z)$, and $\mathcal{J}[\varphi], \mathcal{K}[\varphi]$ are linear operators (often used in this paper) defined as follows:

$$
\begin{aligned}
& \mathcal{J}[\varphi](y, z)=\frac{1}{2} \int_{0}^{y+z} \mathcal{K}[\varphi](x, y, z) \sqrt{x} \mathrm{~d} x, \quad \mathcal{K}[\varphi](x, y, z)=W(x, y, z) \Delta \varphi(x, y, z), \\
& \Delta \varphi(x, y, z)=\varphi(x)+\varphi\left(x_{*}\right)-\varphi(y)-\varphi(z)=(x-y)(x-z) \int_{0}^{1} \int_{0}^{1} \varphi^{\prime \prime}(\xi) \mathrm{d} s \mathrm{~d} t \\
& \xi=y+z-x+t(x-y)+s(x-z), x, y, z \geq 0, x_{*}=(y+z-x)_{+}, \\
& W(x, y, z)=\frac{1}{4 \pi \sqrt{x y z}} \int_{|\sqrt{x}-\sqrt{y}| \vee\left|\sqrt{x_{*}}-\sqrt{z}\right|}^{(\sqrt{x}+\sqrt{y}) \wedge\left(\sqrt{x_{*}}+\sqrt{z}\right)} \mathrm{d} s \int_{0}^{2 \pi} \Phi\left(\sqrt{2} s, \sqrt{2} Y_{*}\right) \mathrm{d} \theta \quad \text { if } \quad x_{*} x y z>0, \\
& W(x, y, z)= \begin{cases}\frac{1}{\sqrt{y z}} \Phi(\sqrt{2 y}, \sqrt{2 z}) & \text { if } \quad x=0, y>0, z>0 \\
\frac{1}{\sqrt{x z}} \Phi(\sqrt{2 x}, \sqrt{2(z-x)}) & \text { if } \quad y=0, z>x>0 \\
\frac{1}{\sqrt{x y}} \Phi(\sqrt{2(y-x)}, \sqrt{2 x}) & \text { if } z=0, y>x>0 \\
0 & \text { others }\end{cases}
\end{aligned}
$$




$$
Y_{*}=Y_{*}(x, y, z, s, \theta)=\left\{\begin{array}{l}
\left|\sqrt{\left(z-\frac{\left(x-y+s^{2}\right)^{2}}{4 s^{2}}\right)_{+}}+e^{\mathrm{i} \theta} \sqrt{\left(x-\frac{\left(x-y+s^{2}\right)^{2}}{4 s^{2}}\right)}\right| \quad \text { if } \quad s>0 \\
0 \quad \text { if } s=0
\end{array}\right.
$$

where $\Phi(r, \rho)$ is given in $(1.5),(u)_{+}=\max \{u, 0\}, a \vee b=\max \{a, b\}, a \wedge b=\min \{a, b\}, \mathrm{i}=\sqrt{-1}$.

Based on the existence results, we introduce directly the concept of measure-valued isotropic solutions of Eq.(1.1) in the weak form:

Definition 1.2. Let $B\left(\mathbf{v}-\mathbf{v}_{*}, \omega\right)$ be given by (1.4), (1.5). Let $F_{0} \in \mathcal{B}_{1}^{+}\left(\mathbb{R}_{\geq 0}\right)$. We say that a family $\left\{F_{t}\right\}_{t \geq 0} \subset \mathcal{B}_{1}^{+}\left(\mathbb{R}_{\geq 0}\right)$, or simply $F_{t}$, is a conservative measure-valued isotropic solution of Eq.(1.1) on the time-interval $[0, \infty)$ with the initial datum $\left.F_{t}\right|_{t=0}=F_{0}$ if

(i) $N\left(F_{t}\right)=N\left(F_{0}\right), E\left(F_{t}\right)=E\left(F_{0}\right)$ for all $t \in[0, \infty)$,

(ii) for every $\varphi \in C_{b}^{2}\left(\mathbb{R}_{\geq 0}\right), t \mapsto \int_{\mathbb{R}_{\geq 0}} \varphi(x) \mathrm{d} F_{t}(x)$ belongs to $C^{1}([0, \infty))$,

(iii) for every $\varphi \in C_{b}^{2}\left(\mathbb{R}_{\geq 0}\right)$

$$
\frac{\mathrm{d}}{\mathrm{d} t} \int_{\mathbb{R}_{\geq 0}} \varphi \mathrm{d} F_{t}=\int_{\mathbb{R}_{\geq 0}^{2}} \mathcal{J}[\varphi] \mathrm{d}^{2} F_{t}+\int_{\mathbb{R}_{\geq 0}^{3}} \mathcal{K}[\varphi] \mathrm{d}^{3} F_{t} \quad \forall t \in[0, \infty) .
$$

Remark 1.3. (1) The transition from (1.19) to (1.20) in defining $W$ is due to the identity

$$
(\sqrt{x}+\sqrt{y}) \wedge\left(\sqrt{x_{*}}+\sqrt{z}\right)-|\sqrt{x}-\sqrt{y}| \vee\left|\sqrt{x_{*}}-\sqrt{z}\right|=2 \min \left\{\sqrt{x}, \sqrt{x_{*}}, \sqrt{y}, \sqrt{z}\right\}
$$

(in case $x_{*}>0$ ) from which one sees also that if $\Phi(r, \rho) \equiv 1$, then $W(x, y, z)$ becomes the function corresponding to the hard sphere model.

(2) As has been proven in [21] that the test function space $C_{b}^{2}\left(\mathbb{R}_{\geq 0}\right)$ in Definition 1.2 can be weaken to

$$
C_{b}^{1,1}\left(\mathbb{R}_{\geq 0}\right)=\left\{\varphi \in C_{b}^{1}\left(\mathbb{R}_{\geq 0}\right) \mid \frac{\mathrm{d}}{\mathrm{d} x} \varphi \in \operatorname{Lip}\left(\mathbb{R}_{\geq 0}\right)\right\}
$$

where $\operatorname{Lip}\left(\mathbb{R}_{\geq 0}\right)$ is the class of functions satisfying Lipschitz condition on $\mathbb{R}_{\geq 0}$. In fact, for the function $\Phi$ satisfying (1.5), it is not difficult to prove that for any $\varphi \in C_{b}^{1,1}\left(\mathbb{R}_{\geq 0}\right)$ the functions $(y, z) \mapsto(1+\sqrt{y}+\sqrt{z})^{-1} \mathcal{J}[\varphi](y, z),(x, y, z) \mapsto \mathcal{K}[\varphi](x, y, z)$ belong to $C_{b}\left(\mathbb{R}_{\geq 0}^{2}\right)$ and $C_{b}\left(\mathbb{R}_{\geq 0}^{3}\right)$ respectively. Thus there is no problem of integrability in the right hand side of Eq.(1.22). A typical example of $\varphi \in C_{b}^{1,1}\left(\mathbb{R}_{\geq 0}\right)$ is $\varphi_{\varepsilon}(x)=\left[(1-x / \varepsilon)_{+}\right]^{2}$ (with $\varepsilon>0$ ) which is often used in Section 5 .

(3) It is proved in Appendix that Definition 1.2 is equivalent to Definition 6.6, the latter is established in [19]. Thus we conclude from Theorem 1 (Weak Stability), Theorem 2 (Existence) and Theorem 3 in [19] that for any $F_{0} \in \mathcal{B}_{1}^{+}\left(\mathbb{R}_{\geq 0}\right)$, the Eq.(1.1) has always a conservative measure-valued isotropic solution $F_{t}$ on the time-interval $[0, \infty)$ with the initial datum $\left.F_{t}\right|_{t=0}=$ 
$F_{0}$. The reason that we use Definition 1.2 is just because the collision integrals in Definition 1.2 are simpler in structure than those in Definition 6.6. Note that if we write $B\left(\mathbf{v}-\mathbf{v}_{*}, \omega\right)$ as

$$
B\left(\mathbf{v}-\mathbf{v}_{*}, \omega\right)=B(V, \cos (\theta))=\frac{1}{(4 \pi)^{2}} V \cos (\theta) \Phi(V \cos (\theta), V \sin (\theta))
$$

where $V=\left|\mathbf{v}-\mathbf{v}_{*}\right|, \theta=\arccos \left(\left|\left(\mathbf{v}-\mathbf{v}_{*}\right) \cdot \omega\right| /\left|\mathbf{v}-\mathbf{v}_{*}\right|\right) \in[0, \pi / 2]$, then it is also required in [19] that $\int_{0}^{\pi / 2} \sin (\theta) B(V, \cos (\theta)) \mathrm{d} \theta>0$ for all $V>0$. But in [19] this strict positivity is just used to guarantee the uniqueness of the equilibrium and to prove (with a further assumption on $B$ ) the moment production; it is apparently not used in the proofs of the three theorems in [19] mentioned above.

Kinetic Temperature. Let $F \in \mathcal{B}_{1}^{+}\left(\mathbb{R}_{\geq 0}\right), N=N(F), E=E(F)$ and suppose $N>0$. If $m$ is the mass of one particle, then $m 4 \pi \sqrt{2} N, m 4 \pi \sqrt{2} E$ are total mass and kinetic energy of the particle system per unite space volume. The kinetic temperature $\bar{T}$ and the kinetic critical temperature $\bar{T}_{c}$ are defined by (see e.g.[19] and references therein)

$$
\bar{T}=\frac{2 m}{3 k_{\mathrm{B}}} \frac{E}{N}, \quad \bar{T}_{c}=\frac{\zeta(5 / 2)}{(2 \pi)^{1 / 3}[\zeta(3 / 2)]^{5 / 3}} \frac{2 m}{k_{\mathrm{B}}} N^{2 / 3}
$$

where $k_{\mathrm{B}}$ is the Boltzmann constant, $\zeta(s)=\sum_{n=1}^{\infty} n^{-s}, s>1$. Some properties involving temperature effect, for instance the Bose-Einstein condensation at low temperature, are often expressed in terms of the ratio

$$
\frac{\bar{T}}{\bar{T}_{c}}=\frac{(2 \pi)^{1 / 3}[\zeta(3 / 2)]^{5 / 3}}{3 \zeta(5 / 2)} \frac{E}{N^{5 / 3}}=2.2720 \frac{E}{N^{5 / 3}} .
$$

Keeping in mind the constant $m 4 \pi \sqrt{2}$, there will be no confusion if we also call $N$ and $E$ the mass and energy of a particle system.

Regular-Singular Decomposition. According to measure theory (see e.g.[28]), every finite positive Borel measure can be uniquely decomposed into regular part and singular part with respect to the Lebesgue measure. For instance if $F \in \mathcal{B}_{1}^{+}\left(\mathbb{R}_{\geq 0}\right)$, then there exist unique $0 \leq f \in L^{1}\left(\mathbb{R}_{\geq 0},(1+x) \sqrt{x} \mathrm{~d} x\right), \nu \in \mathcal{B}_{1}^{+}\left(\mathbb{R}_{\geq 0}\right)$ and a Borel set $Z \subset \mathbb{R}_{\geq 0}$ such that

$$
\mathrm{d} F(x)=f(x) \sqrt{x} \mathrm{~d} x+\mathrm{d} \nu(x), \quad \operatorname{mes}(Z)=0, \quad \nu\left(\mathbb{R}_{\geq 0} \backslash Z\right)=0 .
$$

We call $f$ and $\nu$ the regular part and the singular part of $F$ respectively ${ }^{1}$. If the regular part $f$ is non-zero, i.e., if $\int_{\mathbb{R}_{\geq 0}} f(x) \sqrt{x} \mathrm{~d} x>0$, then we say that $F$ is non-singular.

Bose-Einstein Distribution. According to Theorem 5 of [19] and its equivalent version proved in the Appendix of [21] we know that for any $N>0, E>0$ the Bose-Einstein distribution $F_{\text {be }} \in \mathcal{B}_{1}^{+}\left(\mathbb{R}_{\geq 0}\right)$ given by

$$
\mathrm{d} F_{\mathrm{be}}(x)=f_{\mathrm{be}}(x) \sqrt{x} \mathrm{~d} x+\left(1-\left(\bar{T} / \bar{T}_{c}\right)^{3 / 5}\right)_{+} N \delta(x) \mathrm{d} x
$$

\footnotetext{
${ }^{1}$ Strictly speaking the product $f(x) \sqrt{x}$ is the regular part of $F$. The reason that we only mention $f$ is because $f(x) \sqrt{x}$ comes from the 3D-isotropic function $f=f\left(|\mathbf{v}|^{2} / 2\right)$.
} 
is the unique equilibrium solution of Eq.(1.22) satisfying $N\left(F_{\text {be }}\right)=N, E\left(F_{\text {be }}\right)=E$, where

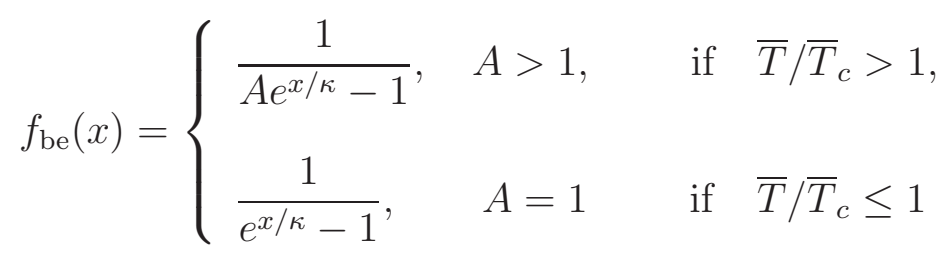

$\delta(x)$ is the Dirac delta function concentrated at $x=0$, i.e. $\delta(x) \mathrm{d} x=\mathrm{d} \nu_{0}(x)$ where $\nu_{0}$ is the Dirac measure concentrated at $x=0$, and functional relations of the coefficients $A=$ $A(N, E), \kappa=\kappa(N, E)$ can be found in for instance Proposition 1 in [20]. From (1.24) one sees that $\bar{T} / \bar{T}_{c} \geq 1 \Longrightarrow \mathrm{d} F_{\text {be }}(x)=f_{\text {be }}(x) \sqrt{x} \mathrm{~d} x$, and

$$
\bar{T} / \bar{T}_{c}<1 \Longrightarrow F_{\mathrm{be}}(\{0\})=\left(1-\left(\bar{T} / \bar{T}_{c}\right)^{3 / 5}\right) N>0
$$

The positive number $\left(1-\left(\bar{T} / \bar{T}_{c}\right)^{3 / 5}\right) N$ is called the Bose-Einstein Condensation (BEC) of the equilibrium state of Bose-Einstein particles at low temperature $\bar{T}<\bar{T}_{c}$.

Entropy. The entropy functional for Eq.(1.1) is

$$
S(f)=\int_{\mathbb{R}^{3}}((1+f(\mathbf{v})) \log (1+f(\mathbf{v}))-f(\mathbf{v}) \log f(\mathbf{v})) \mathrm{d} \mathbf{v}, \quad 0 \leq f \in L_{2}^{1}\left(\mathbb{R}^{3}\right) .
$$

$S(f)$ is always finite since for any $f=f(\mathbf{v}) \geq 0$ we have $([20])$

$$
2 \frac{f}{1+f} \leq(1+f) \log (1+f)-f \log f \leq 2 \min \left\{\sqrt{f},(1+|\mathbf{v}|) f+e^{-|\mathbf{v}| / 2}\right\} .
$$

Moreover since the function

$$
y \mapsto s(y)=(1+y) \log (1+y)-y \log y, \quad y \in[0, \infty)
$$

is concave and non-decreasing with $s(0)=0$, it follows that for all $0 \leq f, g \in L_{2}^{1}\left(\mathbb{R}^{3}\right)$

$$
\begin{aligned}
& 0=S(0) \leq S(f) \leq S(g)<\infty \quad \text { if } \quad f \leq g \\
& S((1-\alpha) f+\alpha g) \geq(1-\alpha) S(f)+\alpha S(g) \quad \text { if } \quad 0<\alpha<1, \\
& S(f+g) \leq S(f)+S(g) .
\end{aligned}
$$

To study measure-valued solutions we define the entropy $S(F)$ of a measure $F \in \mathcal{B}_{2}^{+}\left(\mathbb{R}^{3}\right)$ by

$$
S(F):=\sup _{\left\{f_{n}\right\}_{n=1}^{\infty}} \limsup _{n \rightarrow \infty} S\left(f_{n}\right)
$$

where $\left\{f_{n}\right\}_{n=1}^{\infty}$ under the sup is taken all sequences in $L_{2}^{1}\left(\mathbb{R}^{3}\right)$ satisfying

$$
\begin{aligned}
& f_{n} \geq 0, \quad \sup _{n \geq 1}\left\|f_{n}\right\|_{L_{2}^{1}}<\infty \\
& \lim _{n \rightarrow \infty} \int_{\mathbb{R}^{3}} \psi(\mathbf{v}) f_{n}(\mathbf{v}) \mathrm{d} \mathbf{v}=\int_{\mathbb{R}^{3}} \psi(\mathbf{v}) \mathrm{d} F(\mathbf{v}) \quad \forall \psi \in C_{b}\left(\mathbb{R}^{3}\right) .
\end{aligned}
$$


Let $0 \leq f \in L_{2}^{1}\left(\mathbb{R}^{3}\right)$ be the regular part of $F$, i.e. $\mathrm{d} F(\mathbf{v})=f(\mathbf{v}) \mathrm{d} \mathbf{v}+\mathrm{d} \nu(\mathbf{v})$ with $\nu \geq 0$ the singular part of F. By Lemma 3.2 (see Section 3) we have

$$
S(F)=S(f)
$$

which shows that the singular part of $F$ has no contribution to the entropy $S(F)$ and that $F$ is non-singular if and only if $S(F)>0$. A referee of the paper conveyed us that the convex functions of measures had been defined and studied in [8], and the equality (1.33) coincides with the definition in [8]: $S(F)=\int_{\mathbb{R}^{3}} \mathrm{~d} s(F)(\mathbf{v})$ where $y \mapsto s(y)$ is the function (1.26) and, according to [8], the transformed measure $s(F)$ is defined by $\mathrm{d} s(F)(\mathbf{v})=s(f(\mathbf{v})) \mathrm{d} \mathbf{v}+s_{\infty} \mathrm{d} \nu(\mathbf{v})$ with $s_{\infty}:=\lim _{t \rightarrow+\infty} \frac{s(t)}{t}=0$, here the zero limit is obvious by definition of $s(y)$. Thus we obtain (1.33) again.

For any $0 \leq f \in L^{1}\left(\mathbb{R}_{\geq 0},(1+x) \sqrt{x} \mathrm{~d} x\right)$, the entropy $S(f)$ is defined by $S(f)=S(\bar{f})$ with $\bar{f}(\mathbf{v}):=f\left(|\mathbf{v}|^{2} / 2\right)$, so that (using (1.25) and change of variable)

$$
S(f)=S(\bar{f})=4 \pi \sqrt{2} \int_{\mathbb{R}_{\geq 0}}((1+f(x)) \log (1+f(x))-f(x) \log f(x)) \sqrt{x} \mathrm{~d} x .
$$

In general, the entropy $S(F)$ for a measure $F \in \mathcal{B}_{1}^{+}\left(\mathbb{R}_{\geq 0}\right)$ is defined by $S(F)=S(\bar{F})$ where $\bar{F} \in \mathcal{B}_{2}^{+}\left(\mathbb{R}^{3}\right)$ is defined by $F$ through (1.14) or (1.16) and $S(\bar{F})$ is defined by (1.30). Accordingly for the regular-singular decomposition $\mathrm{d} F(x)=f(x) \sqrt{x} \mathrm{~d} x+\mathrm{d} \nu(x)$ with the singular part $\nu$, we have the regular-singular decomposition $\mathrm{d} \bar{F}(\mathbf{v})=\bar{f}(\mathbf{v}) \mathrm{d} \mathbf{v}+\mathrm{d} \bar{\nu}(\mathbf{v})$ with $\bar{f}(\mathbf{v})=f\left(|\mathbf{v}|^{2} / 2\right)$ and the singular part $\bar{\nu}$ is expressed by $\nu$ through (1.14). Thus from (1.33),(1.34) we have $S(F)=S(\bar{F})=S(\bar{f})=S(f)$.

Although the entropy $S\left(F_{t}\right)$ defined above does not provide any information about the singular part of $F_{t}$ so that one has to consider other methods for proving the convergence of $F_{t}(\{0\})$ to the condensation $F_{\text {be }}(\{0\})$, the entropy difference $S\left(F_{\text {be }}\right)-S\left(F_{t}\right)$ can still describe and control the convergence to equilibrium in a semi-strong norm (see (2.1)), and thus in an indirect way it gives a rate of strong convergence to equilibrium (see proofs of Theorem 5.8 and Theorem 1.4).

Main Result. The main result of the paper is as follows:

Theorem 1.4. Suppose $B\left(\mathbf{v}-\mathbf{v}_{*}, \omega\right)$ satisfy Assumption 1.1 with $0 \leq \eta<1 / 4$. Let $F_{0} \in$ $\mathcal{B}_{1}^{+}\left(\mathbb{R}_{\geq 0}\right)$ satisfy $N\left(F_{0}\right)>0, E\left(F_{0}\right)>0$, let $F_{\text {be }}$ be the unique Bose-Einstein distribution with the same mass $N=N\left(F_{0}\right)$ and energy $E=E\left(F_{0}\right)$. Then there exists a conservative measure-valued isotropic solution $F_{t}$ of Eq.(1.1) on $[0, \infty)$ with the initial datum $F_{0}$ such that $S\left(F_{t}\right) \geq S\left(F_{0}\right)$ and $S\left(F_{t}\right)>0$ for all $t>0$, and for any $0<\lambda<\frac{1}{19}$ it holds

$$
0 \leq S\left(F_{\text {be }}\right)-S\left(F_{t}\right) \leq C(1+t)^{-\lambda}, \quad\left\|F_{t}-F_{\text {be }}\right\|_{1} \leq C(1+t)^{-\frac{(1-\eta) \lambda}{2(4-\eta)}} \quad \forall t \geq 0 .
$$

In particular if $\bar{T} / \bar{T}_{c}<1$ then

$$
\left|F_{t}(\{0\})-\left(1-\left(\bar{T} / \bar{T}_{c}\right)^{3 / 5}\right) N\right| \leq C(1+t)^{-\frac{(1-\eta) \lambda}{2(4-\eta)}} \quad \forall t \geq 0 .
$$


Here the constant $C>0$ depends only on $N, E, b_{0}, \eta$ and $\lambda$.

Remark 1.5. (1) Let $\mathrm{d} F(x)=f(x) \mathrm{d} x+\mathrm{d} \mu(x), \mathrm{d} G(x)=g(x) \mathrm{d} x+\mathrm{d} \nu(x)$ be the regularsingular decompositions of $F, G \in \mathcal{B}^{+}(X)$ respectively. Recall that this means that $0 \leq f, g \in$ $L^{1}(X), \mu, \nu \in \mathcal{B}^{+}(X)$ and there are Borel sets $Z_{F}, Z_{G} \subset X$ such that $\operatorname{mes}\left(Z_{F}\right)=\operatorname{mes}\left(Z_{G}\right)=$ $0, \mu\left(X \backslash Z_{F}\right)=0, \nu\left(X \backslash Z_{G}\right)=0$. According to measure theory, the regular-singular decomposition of $|F-G|$ is

$$
\mathrm{d}|F-G|(x)=|f(x)-g(x)| \mathrm{d} x+\mathrm{d}|\mu-\nu|(x)
$$

i.e.

$$
|F-G|(A)=\int_{A}|f(x)-g(x)| \mathrm{d} x+|\mu-\nu|(A) \quad \forall \text { Borel set } A \subset X
$$

which is easily proved by using the fact that (see e.g.[28]) there is a real Borel function $h$ on $X$ satisfying $h(x)^{2} \equiv 1$ on $X$ such that

$$
\mathrm{d}(F-G)(x)=h(x) \mathrm{d}|F-G|(x), \quad \text { i.e. } \quad \mathrm{d}|F-G|(x)=h(x) \mathrm{d}(F-G)(x) .
$$

The same holds also for $\mu-\nu$. Using (1.36) to the regular-singular decompositions $\mathrm{d} F_{t}(x)=$ $f(x, t) \sqrt{x} \mathrm{~d} x+\mathrm{d} \nu_{t}(x)$ and $\mathrm{d} F_{\text {be }}(x)=f_{\text {be }}(x) \sqrt{x} \mathrm{~d} x+\mathrm{d} \nu_{\text {be }}(x)$ we have

$$
\begin{aligned}
& \left\|F_{t}-F_{\text {be }}\right\|_{1}=\int_{\mathbb{R}_{\geq 0}}(1+x)\left|f(x, t)-f_{\text {be }}(x)\right| \sqrt{x} \mathrm{~d} x+\left\|\nu_{t}-\nu_{\text {be }}\right\|_{1}, \\
& \left\|\nu_{t}-\nu_{\text {be }}\right\|_{1}=\int_{\mathbb{R}_{>0}}(1+x) \mathrm{d} \nu_{t}(x)+\left|F_{t}(\{0\})-F_{\text {be }}(\{0\})\right|
\end{aligned}
$$

for all $t \geq 0$. From these and Theorem 1.4 one sees that, as $t \rightarrow \infty$, the regular part and the singular part of $F_{t}$ converge strongly to the regular part and the singular part of $F_{\text {be }}$ respectively.

(2) In comparison with the exponential convergence to equilibrium for the spatially homogeneous classical Boltzmann equation for hard potentials, the rate of convergence to equilibrium obtained in Theorem 1.4 is very slow even for the hard sphere model (i.e. the case $\eta=0$ ). This is perhaps not only because of the condensation effect (it is hard to obtain a fast decay rate for $\left|F_{t}(\{0\})-F_{\text {be }}(\{0\})\right|$ for low temperature), but also because of the complicated structure of Eq.(1.1) which leads to complicated structures of entropy and entropy dissipation. In fact from [15] one sees that, even for a linearized model of Eq.(1.1) for low temperature, it is difficult to obtain a high order algebraic rate of convergence to equilibrium.

(3) An easy case which is not mentioned in Theorem 1.4 is that $N>0, E=0$. In this case, any conservative measure-valued isotropic solution $F_{t}$ of Eq.(1.1) on $[0, \infty)$ with $N\left(F_{t}\right)=N, E\left(F_{t}\right)=0$ is equal to the same Dirac measure: $F_{t}=F_{\text {be }}$ for all $t \geq 0$, where $\mathrm{d} F_{\text {be }}(x)=N \delta(x) \mathrm{d} x$, and the Dirac measure $F_{\text {be }}$ is also the unique equilibrium solution of Eq.(1.22) satisfying $N(F)=N, E(F)=0$. 
(4) Theorem 1.4 shows that the strong convergence to equilibrium is grossly determined, which means it depends only on the mass, energy, and some constants coming from the collision kernel. In particular, for the case of low temperature $\bar{T} / \bar{T}_{c}<1$, the convergence to BEC does not depend on any local information of initial data. Thus Theorem 1.4 also gives an essential improvement to our previous work [22],[23].

The rest of the paper is organized as follows: In Section 2 we prove an inequality of entropy and entropy dissipation for general functions. In Section 3 we introduce approximate solutions of Eq.(1.1) and prove positive lower bounds of entropy for isotropic approximate solutions and hence for isotropic measure-valued solutions. In Section 4, using the results of Sections 2 and 3 we obtain an algebraic decay rate of the entropy difference $S\left(F_{\mathrm{be}}\right)-S\left(F_{t}\right)$. In Section 5 we prove the long time convergence of the condensation $F_{t}(\{0\})$ to $F_{\text {be }}(\{0\})$, and at the end of that section we finish the proof of our main result Theorem 1.4. Section 6 is an appendix where we prove some general properties of collision integrals and the equivalence of two definitions of measure-valued isotropic solutions of Eq.(1.1), we also prove existence and positivity of some interaction potentials mentioned just above Assumption 1.1.

\section{Inequality of Entropy and Entropy Dissipation}

Entropy and entropy dissipation are powerful tools for investigating long time behavior of solutions of classical and quantum Boltzmann equations. For the latter case, see for instance [10], [20], and the derivation below.

We begin with the following lemma which provides some connections between strong convergence to equilibrium, entropy convergence, and convergence of condensation.

Lemma 2.1. Given $N>0, E>0$. Let $F \in \mathcal{B}_{1}^{+}\left(\mathbb{R}_{\geq 0}\right)$ satisfy $N(F)=N, E(F)=E$. Then there are finite constants $C>0$ (which may be different in different lines) depending only on $N, E$ such that

$$
\frac{1}{C}\left(\left\|F-F_{\text {be }}\right\|_{1}^{\circ}\right)^{2} \leq S\left(F_{\text {be }}\right)-S(F) \leq C\left(\left\|F-F_{\text {be }}\right\|_{1}^{\circ}\right)^{1 / 2}
$$

(II) If $\bar{T} / \bar{T}_{c}<1$, then

$$
\left|F(\{0\})-F_{\text {be }}(\{0\})\right| \leq\left\|F-F_{\text {be }}\right\|_{1} \leq 2\left|F(\{0\})-F_{\text {be }}(\{0\})\right|+C\left(\left\|F-F_{\text {be }}\right\|_{1}^{\circ}\right)^{1 / 3} .
$$

If $\bar{T} / \bar{T}_{c} \geq 1$, then

$$
\left\|F-F_{\text {be }}\right\|_{1} \leq C\left(\left\|F-F_{\text {be }}\right\|_{1}^{\circ}\right)^{1 / 3}
$$

Here

$$
\|\mu\|_{1}^{\circ}=\int_{\mathbb{R}_{\geq 0}} x \mathrm{~d}|\mu|(x), \quad\|\mu\|_{1}=\int_{\mathbb{R}_{\geq 0}}(1+x) \mathrm{d}|\mu|(x), \quad \mu \in \mathcal{B}_{1}\left(\mathbb{R}_{\geq 0}\right) .
$$


Proof. (I): Let $0 \leq f \in L^{1}\left(\mathbb{R}_{+},(1+x) \sqrt{x} \mathrm{~d} x\right), \nu \in \mathcal{B}_{1}^{+}\left(\mathbb{R}_{\geq 0}\right)$ be the regular part and the singular part of $F$. Let $\bar{f}(\mathbf{v})=f\left(|\mathbf{v}|^{2} / 2\right)$ and recall that $\Omega(\mathbf{v})=f_{\text {be }}\left(|\mathbf{v}|^{2} / 2\right)$ is given in (2.14). We have proved in Lemma 4 of [20] that

$$
\frac{1}{C}\left(\|\bar{f}-\Omega\|_{L_{2}^{1}}^{\circ}\right)^{2} \leq S(\Omega)-S(\bar{f}) \leq C\left(\|\bar{f}-\Omega\|_{L_{2}^{1}}^{\circ}\right)^{1 / 2}
$$

where

$$
\|\psi\|_{L_{2}^{1}}^{\circ}=\int_{\mathbb{R}^{3}}|\mathbf{v}|^{2}|\psi(\mathbf{v})| \mathrm{d} \mathbf{v}, \quad \psi \in L_{2}^{1}\left(\mathbb{R}^{3}\right) .
$$

Let $\left(F_{\mathrm{be}}-F\right)^{+}=\frac{1}{2}\left(\left|F_{\mathrm{be}}-F\right|+F_{\mathrm{be}}-F\right)$ be the positive part of $F_{\mathrm{be}}-F$. Then

$$
\begin{aligned}
& \mathrm{d}\left|F_{\mathrm{be}}-F\right|(x)=\mathrm{d} F(x)-\mathrm{d} F_{\mathrm{be}}(x)+2 \mathrm{~d}\left(F_{\mathrm{be}}-F\right)^{+}(x) \\
& \mathrm{d}\left(F_{\mathrm{be}}-F\right)^{+}(x)=h_{+}(x) \mathrm{d}\left(F_{\mathrm{be}}-F\right)(x), \quad h_{+}(x) \in[0,1]
\end{aligned}
$$

where $h_{+}(x)=\frac{1}{2}(1+h(x))$ (see (1.37)). Using $(2.5)$ and $E(F)=E\left(F_{\mathrm{be}}\right)$ we have

$$
\left\|F-F_{\text {be }}\right\|_{1}^{\circ}=2 \int_{\mathbb{R}_{\geq 0}} x \mathrm{~d}\left(F_{\text {be }}-F\right)^{+}(x) \leq 2 \int_{\mathbb{R}_{\geq 0}} x\left|f_{\text {be }}(x)-f(x)\right| \sqrt{x} \mathrm{~d} x .
$$

On the other hand from (1.36)we have

$$
\mathrm{d}\left|F-F_{\text {be }}\right|(x)=\left|f(x)-f_{\text {be }}(x)\right| \sqrt{x} \mathrm{~d} x+\mathrm{d}\left|\nu-\nu_{\text {be }}\right|(x) \geq\left|f(x)-f_{\text {be }}(x)\right| \sqrt{x} \mathrm{~d} x .
$$

Thus

$$
\int_{\mathbb{R}_{\geq 0}} x\left|f(x)-f_{\text {be }}(x)\right| \sqrt{x} \mathrm{~d} x \leq\left\|F-F_{\text {be }}\right\|_{1}^{\circ} \leq 2 \int_{\mathbb{R}_{\geq 0}} x\left|f(x)-f_{\text {be }}(x)\right| \sqrt{x} \mathrm{~d} x .
$$

Since

$$
\int_{\mathbb{R}_{\geq 0}} x\left|f(x)-f_{\mathrm{be}}(x)\right| \sqrt{x} \mathrm{~d} x=\frac{1}{8 \pi \sqrt{2}}\|\bar{f}-\Omega\|_{L_{2}^{1}}^{\circ}, \quad S\left(F_{\mathrm{be}}\right)-S(F)=S(\Omega)-S(\bar{f})
$$

(2.1) follows from (2.4) and (2.7).

(II): For the case $\bar{T} / \bar{T}_{c}<1$, the inequality (2.2) is a result of Lemma 2.8 in [22]. Suppose $\bar{T} / \bar{T}_{c} \geq 1$. Then $\mathrm{d} F_{\text {be }}(x)=f_{\text {be }}(x) \sqrt{x} \mathrm{~d} x=\frac{1}{A e^{x / \kappa}-1} \sqrt{x} \mathrm{~d} x$ and so using $N(F)=N\left(F_{\mathrm{be}}\right)$ and (2.5),(2.6) we have for any $\varepsilon>0$

$$
\begin{aligned}
& \left\|F-F_{\mathrm{be}}\right\|_{0}=2 \int_{[0, \varepsilon]} \mathrm{d}\left(F_{\mathrm{be}}-F\right)^{+}(x)+2 \int_{(\varepsilon, \infty)} \mathrm{d}\left(F_{\mathrm{be}}-F\right)^{+}(x), \\
& \leq 2 \int_{[0, \varepsilon]} \frac{1}{e^{x / \kappa}-1} \sqrt{x} \mathrm{~d} x+\frac{1}{\varepsilon}\left\|F-F_{\mathrm{be}}\right\|_{1}^{\circ} \leq 4 \kappa \sqrt{\varepsilon}+\frac{1}{\varepsilon}\left\|F-F_{\mathrm{be}}\right\|_{1}^{\circ} .
\end{aligned}
$$

Minimizing w.r.t $\varepsilon>0$ gives $\left\|F-F_{\text {be }}\right\|_{0} \leq C\left(\left\|F-F_{\text {be }}\right\|_{1}^{\circ}\right)^{1 / 3}$ and thus $\left\|F-F_{\text {be }}\right\|_{1}=\| F-$ $F_{\text {be }}\left\|_{0}+\right\| F-F_{\text {be }} \|_{1}^{\circ} \leq C\left(\left\|F-F_{\text {be }}\right\|_{1}^{\circ}\right)^{1 / 3}$. 
Since the entropy dissipation $D(f)$ is monotone non-decreasing with respect to the collision kernel $B$, it suffices to establish the relevant estimates for a "minimal" kernel $B_{\min }$ :

$$
B_{\min }\left(\mathbf{v}-\mathbf{v}_{*}, \omega\right)=\frac{b_{0}}{(4 \pi)^{2}} \Psi_{0}\left(\left|\mathbf{v}-\mathbf{v}_{*}\right|\right) \cos ^{3}(\theta) \sin ^{3}(\theta)
$$

where $0<b_{0}<1$ is a constant, $\Psi_{0}: \mathbb{R}_{\geq 0} \rightarrow[0,1]$ is a non-decreasing function satisfying $0<\Psi_{0}(r) \leq 1$ for all $r>0$, and $\theta=\arccos \left(\left|\left(\mathbf{v}-\mathbf{v}_{*}\right) \cdot \omega\right| /\left|\mathbf{v}-\mathbf{v}_{*}\right|\right) \in[0, \pi / 2]$ and we define $\theta=0$ for the case $\mathbf{v}=\mathbf{v}_{*}$. The entropy dissipation corresponding to $B_{\min }$ is

$$
\begin{aligned}
& D_{\min }(f)=\frac{1}{4} \int_{\mathbb{R}^{3} \times \mathbb{R}^{3} \times \mathbb{S}^{2}} B_{\min }\left(\mathbf{v}-\mathbf{v}_{*}, \omega\right) \\
& \times\left(f^{\prime} f_{*}^{\prime}(1+f)\left(1+f_{*}\right)-f f_{*}\left(1+f^{\prime}\right)\left(1+f_{*}^{\prime}\right)\right) \log \left(\frac{f^{\prime} f_{*}^{\prime}(1+f)\left(1+f_{*}\right)}{f f_{*}\left(1+f^{\prime}\right)\left(1+f_{*}^{\prime}\right)}\right) \mathrm{d} \omega \mathrm{d} \mathbf{v} * \mathrm{~d} \mathbf{v} .
\end{aligned}
$$

If we define

$$
\begin{gathered}
\Gamma(a, b)=\left\{\begin{array}{lll}
(a-b) \log \left(\frac{a}{b}\right) & \text { if } & a>0, b>0 \\
\infty & \text { if } & a>0=b \text { or } a=0<b \\
0 & \text { if } \quad a=b=0
\end{array}\right. \\
\Pi(f)=(1+f)\left(1+f_{*}\right)\left(1+f^{\prime}\right)\left(1+f_{*}^{\prime}\right), \quad g=\frac{f}{1+f}, \quad 0 \leq f \in L_{2}^{1}\left(\mathbb{R}^{3}\right)
\end{gathered}
$$

then $D_{\min }(f)$ can be written as a shorter and clear version:

$$
D_{\min }(f)=\frac{1}{4} \int_{\mathbb{R}^{3} \times \mathbb{R}^{3} \times \mathbb{S}^{2}} B_{\min }\left(\mathbf{v}-\mathbf{v}_{*}, \omega\right) \Pi(f) \Gamma\left(g^{\prime} g_{*}^{\prime}, g g_{*}\right) \mathrm{d} \omega \mathrm{d} \mathbf{v}_{*} \mathrm{~d} \mathbf{v} .
$$

We will also use Villani's inequality ([34])

$$
H(f \mid M) \leq \frac{1}{c_{H_{0}}} \mathcal{D}_{2}(f) \quad \forall f \in \mathcal{H}_{0}
$$

where $\mathcal{D}_{2}(f)$ is the entropy dissipation for a "super hard potential" model

$$
\begin{aligned}
& \mathcal{D}_{2}(f)=\frac{1}{4} \int_{\mathbb{R}^{3} \times \mathbb{R}^{3} \times \mathbb{S}^{2}} \frac{2\left|\left(\mathbf{v}-\mathbf{v}_{*}\right) \cdot \omega\right|}{\left|\mathbf{v}-\mathbf{v}_{*}\right|}\left(1+\left|\mathbf{v}-\mathbf{v}_{*}\right|^{2}\right) \Gamma\left(f^{\prime} f_{*}^{\prime}, f f_{*}\right) \mathrm{d} \omega \mathrm{d} \mathbf{v}_{*} \mathrm{~d} \mathbf{v}, \\
& H(f \mid M)=H(f)-H(M), \quad H(f)=\int_{\mathbb{R}^{3}} f(\mathbf{v}) \log f(\mathbf{v}) \mathrm{d} \mathbf{v}, \quad M(\mathbf{v})=\frac{e^{-|\mathbf{v}|^{2} / 2}}{(2 \pi)^{3 / 2}}, \\
& \mathcal{H}_{0}=\left\{0 \leq f \in L_{2}^{1}\left(\mathbb{R}^{3}\right) \mid \int_{\mathbb{R}^{3}}\left(1, \mathbf{v},|\mathbf{v}|^{2} / 3\right) f(\mathbf{v}) \mathrm{d} \mathbf{v}=(1,0,1), H(f \mid M) \leq H_{0}\right\},
\end{aligned}
$$

$0 \leq H_{0}<\infty$ is a constant, and the constant $c_{H_{0}}>0$ depends only $H_{0}$. See [34], see also pp.724-725 of [6] for some estimate of $c_{H_{0}}$. In particular for isotropic functions $f \in \mathcal{H}_{0}$, the inequality (2.12) holds with $c_{H_{0}}=\frac{2 \pi}{7}([34])$. Note that for all $0 \leq f \in L_{2}^{1}\left(\mathbb{R}^{3}\right)$ it holds $\int_{f(\mathbf{v}) \leq 1} f(\mathbf{v})|\log f(\mathbf{v})| \mathrm{d} \mathbf{v}<\infty$ and thus the $H$-function $H(f)$ makes sense and $H(f)<\infty$ if and only if $\int_{\mathbb{R}^{3}} f(\mathbf{v})|\log f(\mathbf{v})| \mathrm{d} \mathbf{v}<\infty$. Note also that in the original Villani's inequality (2.12), 
the entropy dissipation $\mathcal{D}_{2}(f)$ is equivalently expressed with the $\sigma$-representation (4.1) (see the formula (4.2)).

The aim of this section is to prove the following inequality between entropy and entropy dissipation:

Proposition 2.2. Given $N>0, E>0$. Let $f_{\mathrm{be}}(x)=\frac{1}{A e^{x / \kappa}-1}$ be the regular part of the unique equilibrium $F_{\mathrm{be}} \in \mathcal{B}_{1}^{+}\left(\mathbb{R}_{\geq 0}\right)$ which has the mass and energy $N, E$. Let

$$
\Omega(\mathbf{v})=f_{\mathrm{be}}\left(|\mathbf{v}|^{2} / 2\right)=\frac{a e^{-b|\mathbf{v}|^{2}}}{1-a e^{-b|\mathbf{v}|^{2}}}, \quad 0<a=\frac{1}{A} \leq 1, \quad b=\frac{1}{2 \kappa}>0
$$

and let $0 \leq f \in L_{2}^{1}\left(\mathbb{R}^{3}\right)$ satisfy

$$
\int_{\mathbb{R}^{3}}\left(1, \mathbf{v},|\mathbf{v}|^{2} / 2\right) f(\mathbf{v}) \mathrm{d} \mathbf{v}=4 \pi \sqrt{2}(N, 0, E) .
$$

Then $S(f) \leq S(\Omega)$. If in addition $f \in L_{4}^{1}\left(\mathbb{R}^{3}\right)$ and for some $0<S_{0}, C_{0}<\infty$,

$$
S(f) \geq S_{0}, \quad\|f\|_{L_{4}^{1}} \leq C_{0}
$$

then

$$
S(\Omega)-S(f) \leq C\left(\left(\mathcal{D}_{2}(g)\right)^{1 / 10}+\left(D_{\min }(f)\right)^{1 / 4}\right)
$$

where $g=\frac{f}{1+f}, \mathcal{D}_{2}(\cdot), D_{\min }(\cdot)$ are entropy dissipations given in (2.13), (2.8)-(2.11), and the constant $C>0$ depends only on $N, E, S_{0}, C_{0}, b_{0}$ and $\Psi_{0}$.

Proof. From the assumption of the proposition we have $\int_{\mathbb{R}^{3}}|\mathbf{v}|^{2} f(\mathbf{v}) \mathrm{d} \mathbf{v}=\int_{\mathbb{R}^{3}}|\mathbf{v}|^{2} \Omega(\mathbf{v}) \mathrm{d} \mathbf{v}$ and for the case $a=1 / A<1$ we also have $\int_{\mathbb{R}^{3}} f(\mathbf{v}) \mathrm{d} \mathbf{v}=\int_{\mathbb{R}^{3}} \Omega(\mathbf{v}) \mathrm{d} \mathbf{v}$. Thus it follows from Lemma 4 in $[20]$ that $S(f) \leq S(\Omega)$. Now suppose $f \in L_{4}^{1}\left(\mathbb{R}^{3}\right)$. The proof of $(2.15)$ is divided into six steps.

Step1. Let

$$
M_{g}(\mathbf{v})=\alpha e^{-\beta\left|\mathbf{v}-\mathbf{v}_{0}\right|^{2}}, \quad M_{2}(g)=\int_{\mathbb{R}^{3}}\left|\mathbf{v}-\mathbf{v}_{0}\right|^{2} g(\mathbf{v}) \mathrm{d} \mathbf{v}
$$

with $\alpha>0, \beta>0, \mathbf{v}_{0} \in \mathbb{R}^{3}$ given by $g$ through the moment equations

$$
\int_{\mathbb{R}^{3}} M_{g}(\mathbf{v})\left(1, \mathbf{v},|\mathbf{v}|^{2}\right) \mathrm{d} \mathbf{v}=\int_{\mathbb{R}^{3}} g(\mathbf{v})\left(1, \mathbf{v},|\mathbf{v}|^{2}\right) \mathrm{d} \mathbf{v} .
$$

In this step we prove the lower and upper bounds:

$$
C_{1} \leq\|g\|_{L^{1}}, M_{2}(g) \leq C_{2}, \quad C_{3} \leq \alpha, \beta \leq C_{4}, \quad\left|\mathbf{v}_{0}\right| \leq C_{5}
$$

where here and below the constants $0<C_{i}<\infty(i=1,2, \ldots, 16)$ depend only on $N, E, S_{0}, C_{0}, b_{0}$ and $\Psi_{0}$. 
By definition of $S(f)$ and using Cauchy-Schwarz inequality we have

$$
S(f)^{2} \leq\|g\|_{L^{1}} \int_{f(\mathbf{v})>0}\left(1+\frac{1}{f(\mathbf{v})}\right) s(f(\mathbf{v}))^{2} \mathrm{~d} \mathbf{v} .
$$

Then we use the inequality $\log (1+y) \leq \sqrt{y} \wedge y$ to get for all $y>0$

$$
s(y)^{2}=\left(\log (1+y)+y \log \left(1+\frac{1}{y}\right)\right)^{2} \leq \min \left\{4 y, 2 y^{2}+2 y^{2}\left(\log \left(1+\frac{1}{y}\right)\right)^{2}\right\}
$$

from which we deduce

$$
\int_{f(\mathbf{v})>0}\left(1+\frac{1}{f(\mathbf{v})}\right) s(f(\mathbf{v}))^{2} \mathrm{~d} \mathbf{v} \leq 6\|f\|_{L^{1}}+2 \int_{f(\mathbf{v})>0} f(\mathbf{v})\left(\log \left(1+\frac{1}{f(\mathbf{v})}\right)\right)^{2} \mathrm{~d} \mathbf{v}
$$

Furthermore using the inequality $y\left(\log \left(1+\frac{1}{y}\right)\right)^{2} \leq 4 \sqrt{y}(y>0)$ we have

$$
\begin{aligned}
f(\mathbf{v})\left(\log \left(1+\frac{1}{f(\mathbf{v})}\right)\right)^{2} & \leq 4 \sqrt{f(\mathbf{v})} 1_{\left\{f(\mathbf{v}) \leq e^{-|\mathbf{v}|\}}\right.}+f(\mathbf{v})\left(\log \left(1+e^{|\mathbf{v}|}\right)\right)^{2} 1_{\left\{f(\mathbf{v})>e^{-|\mathbf{v}|}\right\}} \\
& \leq 4 e^{-|\mathbf{v}| / 2}+2 f(\mathbf{v})\langle\mathbf{v}\rangle^{2}
\end{aligned}
$$

Thus

$$
\int_{f(\mathbf{v})>0} f(\mathbf{v})\left(\log \left(1+\frac{1}{f(\mathbf{v})}\right)\right)^{2} \mathrm{~d} \mathbf{v} \leq 2^{8} \pi+2\|f\|_{L_{2}^{1}}
$$

which together with (2.18), (2.19) gives

$$
\|f\|_{L^{1}} \geq\|g\|_{L^{1}} \geq \frac{S(f)^{2}}{2^{9} \pi+10\|f\|_{L_{2}^{1}}} .
$$

Next using Cauchy-Schwarz inequality and $0 \leq g \leq 1$ we have

$$
\begin{array}{cc}
\quad\left(\|g\|_{L^{1}}\right)^{2} \leq & \int_{\mathbb{R}^{3}}\left|\mathbf{v}-\mathbf{v}_{0}\right|^{2} g(\mathbf{v}) \mathrm{d} \mathbf{v} \int_{\mathbb{R}^{3}} \frac{1}{\left|\mathbf{v}-\mathbf{v}_{0}\right|^{2}} g(\mathbf{v}) \mathrm{d} \mathbf{v} \leq M_{2}(g) 2(4 \pi)^{2 / 3}\left(\|g\|_{L^{1}}\right)^{1 / 3} \\
& M_{2}(g) \geq \frac{1}{2(4 \pi)^{2 / 3}}\left(\|g\|_{L^{1}}\right)^{5 / 3} \geq \frac{1}{2(4 \pi)^{2 / 3}}\left(\frac{S(f)^{2}}{2^{9} \pi+10\|f\|_{L_{2}^{1}}}\right)^{5 / 3} .
\end{array}
$$

Finally from $\mathbf{v}_{0}=\frac{1}{\|g\|_{L^{1}}} \int_{\mathbb{R}^{3}} \mathbf{v} g(\mathbf{v}) \mathrm{d} \mathbf{v}$ and Cauchy-Schwarz inequality we have

$$
\left|\mathbf{v}_{0}\right|^{2} \leq \frac{1}{\|g\|_{L^{1}}} \int_{\mathbb{R}^{3}}|\mathbf{v}|^{2} g(\mathbf{v}) \mathrm{d} \mathbf{v} \quad \text { hence } \quad M_{2}(g) \leq 4 \int_{\mathbb{R}^{3}}|\mathbf{v}|^{2} g(\mathbf{v}) \mathrm{d} \mathbf{v} \leq 4 \int_{\mathbb{R}^{3}}|\mathbf{v}|^{2} f(\mathbf{v}) \mathrm{d} \mathbf{v}
$$

Since, from the moment equation (2.16),

$$
\alpha=\pi^{-3 / 2}\left(\frac{3}{2}\right)^{3 / 2} \frac{\left(\|g\|_{L^{1}}\right)^{5 / 2}}{\left(M_{2}(g)\right)^{3 / 2}}, \quad \beta=\frac{3}{2} \frac{\|g\|_{L^{1}}}{M_{2}(g)}
$$

the inequalities in (2.17) follow from (2.20)-(2.23), $\|f\|_{L^{1}}=4 \pi \sqrt{2} N, \int_{\mathbb{R}^{3}}|\mathbf{v}|^{2} f(\mathbf{v}) \mathrm{d} \mathbf{v}=8 \pi \sqrt{2} E$, and $S(f) \geq S_{0}$. 
Step2. Let

$$
M_{g}^{*}(\mathbf{v})=(\alpha \wedge 1) e^{-\beta\left|\mathbf{v}-\mathbf{v}_{0}\right|^{2}}, \quad \Omega_{g}^{*}(\mathbf{v})=\frac{M_{g}^{*}(\mathbf{v})}{1-M_{g}^{*}(\mathbf{v})} .
$$

According to Proposition 2 in $[20]\left(\right.$ taking $\underline{b}(\cos (\theta))=\frac{b_{0}}{(4 \pi)^{2}}, \Psi(r)=\Psi_{0}(r)$ in that proposition) we have

$$
\left\|\left(1-M_{g}^{*}\right)\left(f-\Omega_{g}^{*}\right)\right\|_{L^{1}} \leq C_{\alpha, \beta}\left(\|f\|_{L^{1}}+\left\|M_{g}^{*}\right\|_{L^{1}}\right)\left(\left\|g-M_{g}^{*}\right\|_{L^{1}}+\sqrt{D_{\min }(f)}\right)
$$

where

$$
C_{\alpha, \beta}=C_{\alpha, \beta, b_{0}, \eta}=C_{b_{0}}\left(\int_{\mathbb{R}^{3}}(\alpha \wedge 1) e^{-\beta|\mathbf{z}|^{2}} \Psi_{0}(|\mathbf{z}|) \mathrm{d} \mathbf{z}\right)^{-1}
$$

$C_{b_{0}}>0$ depends only on $b_{0}$.

Step3. Here we prove that

$$
S(\Omega)-S\left(\Omega_{g}^{*}\right) \leq C_{6}\left(\left\|g-M_{g}^{*}\right\|_{L^{1}}+\sqrt{D_{\min }(f)}\right)^{1 / 2} .
$$

First from the assumption of the proposition we have

$$
\int_{\mathbb{R}^{3}} \Omega(\mathbf{v}) \mathrm{d} \mathbf{v} \leq \int_{\mathbb{R}^{3}} f(\mathbf{v}) \mathrm{d} \mathbf{v}, \int_{\mathbb{R}^{3}}\left(\mathbf{v},|\mathbf{v}|^{2} / 2\right) \Omega(\mathbf{v}) \mathrm{d} \mathbf{v}=\int_{\mathbb{R}^{3}}\left(\mathbf{v},|\mathbf{v}|^{2} / 2\right) f(\mathbf{v}) \mathrm{d} \mathbf{v} .
$$

Since the function $y \mapsto s(y)$ given in 1.26 is concave, it holds the inequality $s(y)-s\left(y_{0}\right) \leq$ $\left(\log \left(1+\frac{1}{y_{0}}\right)\right)\left(y-y_{0}\right), y \geq 0, y_{0}>0$, from which and $(2.26)$ we obtain

$$
\begin{aligned}
S(\Omega)-S\left(\Omega_{g}^{*}\right) & \leq \int_{\mathbb{R}^{3}}\left(\log \left(\frac{1}{\alpha \wedge 1}\right)+\beta\left|\mathbf{v}-\mathbf{v}_{0}\right|^{2}\right)\left(\Omega(\mathbf{v})-\Omega_{g}^{*}(\mathbf{v})\right) \mathrm{d} \mathbf{v} \\
& \leq \int_{\mathbb{R}^{3}}\left(\log \left(\frac{1}{\alpha \wedge 1}\right)+\beta\left|\mathbf{v}-\mathbf{v}_{0}\right|^{2}\right)\left(f(\mathbf{v})-\Omega_{g}^{*}(\mathbf{v})\right) \mathrm{d} \mathbf{v} .
\end{aligned}
$$

Next using the inequalities

$$
\log \left(\frac{1}{\alpha \wedge 1}\right) \leq \frac{1-\alpha \wedge 1}{\alpha \wedge 1}, \quad \frac{1-\alpha \wedge 1+\beta\left|\mathbf{v}-\mathbf{v}_{0}\right|^{2}}{1+\beta\left|\mathbf{v}-\mathbf{v}_{0}\right|^{2}} \leq 1-M_{g}^{*}(\mathbf{v})
$$

it is easily seen that

$$
\log \left(\frac{1}{\alpha \wedge 1}\right)+\beta\left|\mathbf{v}-\mathbf{v}_{0}\right|^{2} \leq \frac{1}{\alpha \wedge 1}\left(1-M_{g}^{*}(\mathbf{v})\right)\left(1+\beta\left|\mathbf{v}-\mathbf{v}_{0}\right|^{2}\right) .
$$

From this, Cauchy-Schwarz inequality, and $0 \leq 1-M_{g}^{*} \leq 1$ we obtain

$$
\begin{aligned}
& \int_{\mathbb{R}^{3}}\left(\log \left(\frac{1}{\alpha \wedge 1}\right)+\beta\left|\mathbf{v}-\mathbf{v}_{0}\right|^{2}\right)\left|f(\mathbf{v})-\Omega_{g}^{*}(\mathbf{v})\right| \mathrm{d} \mathbf{v} \\
& \leq \int_{\mathbb{R}^{3}} \frac{1}{\alpha \wedge 1}\left(1-M_{g}^{*}(\mathbf{v})\right)\left(1+\beta\left|\mathbf{v}-\mathbf{v}_{0}\right|^{2}\right)\left|f(\mathbf{v})-\Omega_{g}^{*}(\mathbf{v})\right| \mathrm{d} \mathbf{v} \\
& =\frac{\sqrt{2\left(\beta^{2} \vee 1\right)}}{\alpha \wedge 1}\left(\left\|\left(1-M_{g}^{*}\right)\left(f-\Omega_{g}^{*}\right)\right\|_{L^{1}}\right)^{1 / 2}\left(\int_{\mathbb{R}^{3}}\left(1+\left|\mathbf{v}-\mathbf{v}_{0}\right|^{4}\right)\left|f(\mathbf{v})-\Omega_{g}^{*}(\mathbf{v})\right| \mathrm{d} \mathbf{v}\right)^{1 / 2} \\
& \leq C_{7}\left(\left\|g-M_{g}^{*}\right\|_{L^{1}}+\sqrt{D_{\min }(f)}\right)^{1 / 2}
\end{aligned}
$$


where the constant $C_{7}$ comes from (2.24), $\|f\|_{L_{4}^{1}} \leq C_{0},\left|\mathbf{v}_{0}\right| \leq C_{5}$, and

$$
\int_{\mathbb{R}^{3}}\left(1+\left|\mathbf{v}-\mathbf{v}_{0}\right|^{4}\right) \Omega_{g}^{*}(\mathbf{v}) \mathrm{d} \mathbf{v} \leq 4 \pi \int_{0}^{\infty} r^{2}\left(1+r^{4}\right) \frac{1}{e^{\beta r^{2}}-1} \mathrm{~d} r<\infty .
$$

Combining (2.27) and (2.29) we obtain (2.25).

Step4. We next prove that

$$
S\left(\Omega_{g}^{*}\right)-S(f) \leq C_{8}\left(\left\|g-M_{g}^{*}\right\|_{L^{1}}+\sqrt{D_{\min }(f)}\right)^{1 / 2} .
$$

It is easily seen from $s^{\prime}(y)=\log (1+1 / y) \leq 3 y^{-1 / 3}$ that for all $y>y_{0} \geq 0$

$$
s(y)-s\left(y_{0}\right)=\left(y-y_{0}\right) \int_{0}^{1} \log \left(1+\frac{1}{y_{0}+\theta\left(y-y_{0}\right)}\right) \mathrm{d} \theta \leq 5\left(y-y_{0}\right)^{2 / 3} .
$$

From these and Cauchy-Schwarz inequality we have

$$
\begin{aligned}
& S\left(\Omega_{g}^{*}\right)-S(f) \\
& \leq \int_{\Omega_{g}^{*}(\mathbf{v})>f(\mathbf{v})}\left[s\left(\Omega_{g}^{*}(\mathbf{v})\right)-s(f(\mathbf{v}))\right] \mathrm{d} \mathbf{v} \leq 5 \int_{\Omega_{g}^{*}(\mathbf{v})>f(\mathbf{v})}\left(\Omega_{g}^{*}(\mathbf{v})-f(\mathbf{v})\right)^{2 / 3} \mathrm{~d} \mathbf{v} \\
& \leq 5\left(\left\|\left(1-M_{g}^{*}\right)\left(\Omega_{g}^{*}-f\right)\right\|_{L^{1}}\right)^{1 / 2}\left(\int_{\mathbb{R}^{3}} \frac{1}{1-M_{g}^{*}(\mathbf{v})}\left(\Omega_{g}^{*}(\mathbf{v})\right)^{1 / 3} \mathrm{~d} \mathbf{v}\right)^{1 / 2} .
\end{aligned}
$$

From the second inequality in (2.28) we have $\frac{1}{1-M_{g}^{*}(\mathbf{v})} \leq \frac{1}{\beta\left|\mathbf{v}-\mathbf{v}_{0}\right|^{2}}\left(1+\beta\left|\mathbf{v}-\mathbf{v}_{0}\right|^{2}\right)$, so the last integral in (2.32) is finite (using change of variable)

$$
\begin{aligned}
& \int_{\mathbb{R}^{3}} \frac{1}{1-M_{g}^{*}(\mathbf{v})}\left(\Omega_{g}^{*}(\mathbf{v})\right)^{1 / 3} \mathrm{~d} \mathbf{v}=\int_{\mathbb{R}^{3}} \frac{\left(M_{g}^{*}(\mathbf{v})\right)^{1 / 3}}{\left(1-M_{g}^{*}(\mathbf{v})\right)^{4 / 3}} \mathrm{~d} \mathbf{v} \\
& \leq 4 \pi(1 / \beta)^{4 / 3} \int_{0}^{\infty} \frac{1}{r^{2 / 3}}\left(1+\beta r^{2}\right)^{4 / 3}\left((\alpha \wedge 1) e^{-\beta r^{2}}\right)^{1 / 3} \mathrm{~d} r \leq C_{9} .
\end{aligned}
$$

The inequality (2.30) then follows from this, (2.32) and (2.24).

Step5. Now we prove that

$$
S(\Omega)-S(f) \leq C_{10}\left(\left(\left\|g-M_{g}\right\|_{L^{1}}\right)^{1 / 5}+\left(D_{\min }(f)\right)^{1 / 4}\right) .
$$

In fact first we have from (2.25) and (2.30) that

$$
S(\Omega)-S(f) \leq C_{11}\left(\left\|g-M_{g}^{*}\right\|_{L^{1}}+\sqrt{D_{\min }(f)}\right)^{1 / 2} .
$$

If $\alpha \leq 1$, then $M_{g}^{*}=M_{g}$ and so from (2.34) we see that (2.33) holds.

Suppose $\alpha>1$. From $\|g\|_{L^{1}}=\left\|M_{g}\right\|_{L^{1}}$ and $0 \leq g(\mathbf{v}) \leq 1$ we have

$$
\left\|g-M_{g}\right\|_{L^{1}}=2 \int_{\mathbb{R}^{3}}\left(M_{g}(\mathbf{v})-g(\mathbf{v})\right)_{+} \mathrm{d} \mathbf{v} \geq 8 \pi \int_{0}^{\infty} r^{2}\left(\alpha e^{-\beta r^{2}}-1\right)_{+} \mathrm{d} r
$$


and using the inequality $\alpha e^{-\beta r^{2}}-1 \geq \alpha \beta\left(\frac{\alpha-1}{\alpha \beta}-r^{2}\right)$ to the last term we have

$$
8 \pi \int_{0}^{\infty} r^{2}\left(\alpha e^{-\beta r^{2}}-1\right)_{+} \mathrm{d} r \geq 8 \pi \int_{0}^{\infty} r^{2} \alpha \beta\left(\frac{\alpha-1}{\alpha \beta}-r^{2}\right)_{+} \mathrm{d} r=\frac{16 \pi}{15} \frac{(\alpha-1)^{5 / 2}}{(\alpha \beta)^{3 / 2}}
$$

so that we deduce

$$
\begin{aligned}
& \alpha-1 \leq\left(\frac{15}{16 \pi}(\alpha \beta)^{3 / 2}\right)^{2 / 5}\left(\left\|g-M_{g}\right\|_{L^{1}}\right)^{2 / 5}, \\
& \left\|M_{g}-M_{g}^{*}\right\|_{L^{1}}=(\alpha-1) \int_{\mathbb{R}^{3}} e^{-\beta\left|\mathbf{v}-\mathbf{v}_{0}\right|^{2}} \mathrm{~d} \mathbf{v} \leq C_{12}\left(\left\|g-M_{g}\right\|_{L^{1}}\right)^{2 / 5}, \\
& \left\|g-M_{g}^{*}\right\|_{L^{1}} \leq\left\|g-M_{g}\right\|_{L^{1}}+\left\|M_{g}-M_{g}^{*}\right\|_{L^{1}} \leq C_{13}\left(\left\|g-M_{g}\right\|_{L^{1}}\right)^{2 / 5} .
\end{aligned}
$$

Inserting $\left\|g-M_{g}^{*}\right\|_{L^{1}} \leq C_{13}\left(\left\|g-M_{g}\right\|_{L^{1}}\right)^{2 / 5}$ into the right hand side of (2.34) gives (2.33).

Step6. We will use Csiszar-Kullback inequality

$$
\left\|g-M_{g}\right\|_{L^{1}} \leq \sqrt{2\|g\|_{L^{1}}} \sqrt{H\left(g \mid M_{g}\right)}
$$

and Villani's inequality (2.12) to finish the proof of (2.15). To do this we need to normalize $g, M_{g}$. Let

$$
\widetilde{g}(\mathbf{v})=\mu g\left(\lambda \mathbf{v}+\mathbf{v}_{0}\right), \quad \widetilde{M}_{g}(\mathbf{v})=\mu M_{g}\left(\lambda \mathbf{v}+\mathbf{v}_{0}\right)=\mu \alpha e^{-\lambda \beta|\mathbf{v}|^{2}}
$$

where

$$
\lambda=3^{-1 / 2}\left(\frac{M_{2}(g)}{\|g\|_{L^{1}}}\right)^{1 / 2}, \quad \mu=3^{-3 / 2} \frac{\left(M_{2}(g)\right)^{3 / 2}}{\left(\|g\|_{L^{1}}\right)^{5 / 2}} .
$$

Then $\widetilde{M}_{g}(\mathbf{v})=M(\mathbf{v})=(2 \pi)^{-3 / 2} e^{-|\mathbf{v}|^{2} / 2}$ and $\widetilde{g} \in \mathcal{H}_{0}$ with the constant

$$
H_{0}=\log \left(\frac{\left(C_{2}\right)^{3 / 2}}{3^{3 / 2}\left(C_{1}\right)^{5 / 2}}\right)+\frac{3}{2} \log (2 \pi e)
$$

where $C_{1}, C_{2}$ are the constants in (2.17). In fact from (2.36) and $0 \leq g \leq 1$ we have $\lambda^{-3} \mu\|g\|_{L^{1}}=$ 1 and $H(g) \leq 0$, and so $0 \leq H\left(\widetilde{g} \mid \widetilde{M}_{g}\right) \leq \log (\mu)-H\left(\widetilde{M}_{g}\right) \leq H_{0}$. Applying Villani's inequality (2.12) to $\widetilde{g}, \widetilde{M}_{g}$ we have

$$
\frac{1}{\|g\|_{L^{1}}} H\left(g \mid M_{g}\right)=H\left(\widetilde{g} \mid \widetilde{M}_{g}\right) \leq \frac{1}{c_{H_{0}}} \mathcal{D}_{2}(\widetilde{g})
$$

Since

$$
\begin{aligned}
\mathcal{D}_{2}(\widetilde{g}) & =\frac{1}{4} \int_{\mathbb{R}^{3} \times \mathbb{R}^{3} \times \mathbb{S}^{2}} \frac{2\left|\left(\mathbf{v}-\mathbf{v}_{*}\right) \cdot \omega\right|}{\left|\mathbf{v}-\mathbf{v}_{*}\right|}\left(1+\left|\mathbf{v}-\mathbf{v}_{*}\right|^{2}\right) \Gamma\left(\widetilde{g}^{\prime} \widetilde{g}_{*}^{\prime}, \widetilde{g} \widetilde{g}_{*}\right) \mathrm{d} \mathbf{v} \mathrm{d} \mathbf{v}_{*} \mathrm{~d} \omega \\
& =\frac{\mu^{2}}{\lambda^{8}} \frac{1}{4} \int_{\mathbb{R}^{3} \times \mathbb{R}^{3} \times \mathbb{S}^{2}} \frac{2\left|\left(\mathbf{v}-\mathbf{v}_{*}\right) \cdot \omega\right|}{\left|\mathbf{v}-\mathbf{v}_{*}\right|}\left(\lambda^{2}+\left|\mathbf{v}-\mathbf{v}_{*}\right|^{2}\right) \Gamma\left(g^{\prime} g_{*}^{\prime}, g g_{*}\right) \mathrm{d} \mathbf{v} \mathrm{d} \mathbf{v}_{*} \mathrm{~d} \omega \\
& \leq \frac{\mu^{2}}{\lambda^{8}} \max \left\{\lambda^{2}, 1\right\} \mathcal{D}_{2}(g) \leq C_{14} \mathcal{D}_{2}(g),
\end{aligned}
$$

it follows from $(2.35,(2.37)$ that

$$
\left\|g-M_{g}\right\|_{L^{1}} \leq \sqrt{2}\|g\|_{L^{1}} \sqrt{H\left(\widetilde{g} \mid \widetilde{M}_{g}\right)} \leq C_{15} \sqrt{\mathcal{D}_{2}(g)}
$$

Inserting this into (2.33) gives (2.15) with $C=C_{10} \max \left\{\left(C_{15}\right)^{1 / 5}, 1\right\}$ and completes the proof. 


\section{Positive Lower Bound of Entropy}

In this section we prove that if an initial datum $F_{0} \in \mathcal{B}_{1}^{+}\left(\mathbb{R}_{\geq 0}\right)$ has positive energy, $E\left(F_{0}\right)>$ 0 , then there is a conservative measure-valued solution $F_{t}$ of Eq. with $F_{t=0}=F_{0}$, such that $S\left(F_{t}\right)>0$ for all $t>0$. This is equivalent to saying that $F_{t}$ is non-singular for all $t>0$ even if $F_{0}$ is singular. To do this we first prove that the entropies of isotropic approximate solutions have a uniform positive lower bound. For convenience of stating approximate solutions under consideration, we introduce a definition of a class of approximate solutions:

Definition 3.1. Let $B\left(\mathbf{v}-\mathbf{v}_{*}, \omega\right)$ be given by (1.4), (1.5). We say that $\left\{B_{K}\left(\mathbf{v}-\mathbf{v}_{*}, \omega\right)\right\}_{K \in \mathbb{N}}$ is a sequence of approximation of $B\left(\mathbf{v}-\mathbf{v}_{*}, \omega\right)$ if $B_{K}\left(\mathbf{v}-\mathbf{v}_{*}, \omega\right)$ are such Borel measurable functions on $\mathbb{R}^{3} \times \mathbb{S}^{2}$ that they are functions of $\left(\left|\mathbf{v}-\mathbf{v}^{\prime}\right|,\left|\mathbf{v}-\mathbf{v}_{*}^{\prime}\right|\right)$ only and satisfy

$$
B_{K}\left(\mathbf{v}-\mathbf{v}_{*}, \omega\right) \geq 0, \quad \lim _{K \rightarrow \infty} B_{K}\left(\mathbf{v}-\mathbf{v}_{*}, \omega\right)=B\left(\mathbf{v}-\mathbf{v}_{*}, \omega\right)
$$

for all $\left(\mathbf{v}-\mathbf{v}_{*}, \omega\right) \in \mathbb{R}^{3} \times \mathbb{S}^{2}$. Let $Q_{K}(f)$ be the collision integral operators corresponding to the approximate kernels $B_{K}$, i.e.

$$
Q_{K}(f)(\mathbf{v})=\int_{\mathbb{R}^{3} \times \mathbb{S}^{2}} B_{K}\left(\mathbf{v}-\mathbf{v}_{*}, \omega\right)\left(f^{\prime} f_{*}^{\prime}\left(1+f+f_{*}\right)-f f_{*}\left(1+f^{\prime}+f_{*}^{\prime}\right)\right) \mathrm{d} \omega \mathrm{d} \mathbf{v}_{*} .
$$

Given any $K \in \mathbb{N}$ and $0 \leq f_{0}^{K} \in L_{2}^{1}\left(\mathbb{R}^{3}\right)$. We say that $f^{K}=f^{K}(\mathbf{v}, t)$ is a conservative approximate solution of Eq.(1.1) on $\mathbb{R}^{3} \times[0, \infty)$ corresponding to the approximate kernel $B_{K}$ with the initial datum $f_{0}^{K}$ if $(\mathbf{v}, t) \mapsto f^{K}(\mathbf{v}, t)$ is a nonnegative Lebesgue measurable function on $\mathbb{R}^{3} \times[0, \infty)$ satisfying

(i) $\sup _{t \geq 0}\left\|f^{K}(t)\right\|_{L_{2}^{1}}<\infty$ (here and below $f^{K}(t):=f^{K}(\cdot, t)$ ) and

$$
\int_{0}^{T} \mathrm{~d} t \int_{\mathbb{R}^{3} \times \mathbb{R}^{3} \times \mathbb{S}^{2}} B_{K}\left(\mathbf{v}-\mathbf{v}_{*}, \omega\right)\left(f^{K}\right)^{\prime}\left(f^{K}\right)_{*}^{\prime}\left(1+f^{K}+f_{*}^{K}\right) \sqrt{1+|\mathbf{v}|^{2}+\left|\mathbf{v}_{*}\right|^{2}} \mathrm{~d} \omega \mathrm{d} \mathbf{v} \mathrm{d} \mathbf{v}_{*}<\infty
$$

for all $0<T<\infty$.

(ii) There is a null set $Z \subset \mathbb{R}^{3}$ which is independent of $t$ such that

$$
f^{K}(\mathbf{v}, t)=f_{0}^{K}(\mathbf{v})+\int_{0}^{t} Q_{K}\left(f^{K}\right)(\mathbf{v}, \tau) \mathrm{d} \tau \quad \forall t \in[0, \infty), \forall \mathbf{v} \in \mathbb{R}^{3} \backslash Z .
$$

(iii) $f^{K}$ conserves the mass, momentum, and energy, and satisfies the entropy equality, i.e.

$$
\begin{array}{ll}
\int_{\mathbb{R}^{3}}\left(1, \mathbf{v},|\mathbf{v}|^{2} / 2\right) f^{K}(\mathbf{v}, t) \mathrm{d} \mathbf{v}=\int_{\mathbb{R}^{3}}\left(1, \mathbf{v},|\mathbf{v}|^{2} / 2\right) f_{0}^{K}(\mathbf{v}) \mathrm{d} \mathbf{v} \quad \forall t \geq 0 \\
S\left(f^{K}(t)\right)=S\left(f_{0}^{K}\right)+\int_{0}^{t} D_{K}\left(f^{K}(\tau)\right) \mathrm{d} \tau \quad \forall t \geq 0 .
\end{array}
$$

Here $Q_{K}\left(f^{K}\right)(\mathbf{v}, t)=Q_{K}\left(f^{K}(\cdot, t)\right)(\mathbf{v}), D_{K}(f)$ is the entropy dissipation corresponding to the approximate kernel $B_{K}\left(\mathbf{v}-\mathbf{v}_{*}, \omega\right)$ defined as in (2.9)-(2.11), i.e.

$$
D_{K}(f)=\frac{1}{4} \int_{\mathbb{R}^{3} \times \mathbb{R}^{3} \times \mathbb{S}^{2}} B_{K}\left(\mathbf{v}-\mathbf{v}_{*}, \omega\right) \Pi(f) \Gamma\left(g^{\prime} g_{*}^{\prime}, g g_{*}\right) \mathrm{d} \omega \mathrm{d} \mathbf{v}_{*} \mathrm{~d} \mathbf{v}
$$


with $g=f /(1+f)$.

If a conservative approximate solution $f^{K}$ is isotropic, i.e. if $f^{K}(\mathbf{v}, t) \equiv f^{K}\left(|\mathbf{v}|^{2} / 2, t\right)$, then $f^{K}$ is called a conservative isotropic approximate solution of Eq.(1.1).

By using change of variables one sees that the integral in the left hand side of (3.1) is equal to that where $\left(f^{K}\right)^{\prime}\left(f^{K}\right)_{*}^{\prime}\left(1+f^{K}+f_{*}^{K}\right)$ is replaced by $f^{K} f_{*}^{K}\left(1+\left(f^{K}\right)^{\prime}+\left(f^{K}\right)_{*}^{\prime}\right)$. Thus (3.1) not only implies $Q_{K}\left(f^{K}\right) \in L^{1}\left(\mathbb{R}^{3} \times[0, T]\right)$ for all $0<T<\infty$ so that the integral in right hand said of 3.2) is absolutely convergent for all $\mathbf{v} \in \mathbb{R}^{3} \backslash Z$ and all $t \geq 0$, but also enables us to prove some important relations between entropy and entropy dissipation for approximate solutions as we will do in the proof of Proposition 4.2 below.

Also as one sees from (3.1) that a main role of an approximation $B_{K}$ of $B$ is to ensure the absolute convergence of the cubic collision integrals. A suitable class of such $B_{K}$ that had been used before is

$$
B_{K}\left(\mathbf{v}-\mathbf{v}_{*}, \omega\right)=\min \left\{B\left(\mathbf{v}-\mathbf{v}_{*}, \omega\right), K\left|\mathbf{v}-\mathbf{v}^{\prime}\right|^{2}\left|\mathbf{v}-\mathbf{v}_{*}^{\prime}\right|\right\}, \quad K \geq 1
$$

which works well at least for isotropic approximate solutions (see e.g.[18]). In fact using $\sqrt{1+|\mathbf{v}|^{2}+\left|\mathbf{v}_{*}\right|^{2}} \leq\langle\mathbf{v}\rangle\left\langle\mathbf{v}_{*}\right\rangle$ one has, as proved in [18], that for all isotropic functions $f, g \in$ $L_{1}^{1}\left(\mathbb{R}^{3}\right), h \in L^{1}\left(\mathbb{R}^{3}\right)$ (recall that $f$ is isotropic means that $f(\mathbf{v})$ depends only on $|\mathbf{v}|$ )

$$
\begin{aligned}
& \int_{\mathbb{R}^{3} \times \mathbb{R}^{3} \times \mathbb{S}^{2}} B_{K}\left(\mathbf{v}-\mathbf{v}_{*}, \omega\right)\left|f\left(\mathbf{v}^{\prime}\right) g\left(\mathbf{v}_{*}^{\prime}\right) h(\mathbf{v})\right| \sqrt{1+|\mathbf{v}|^{2}+\left|\mathbf{v}_{*}\right|^{2}} \mathrm{~d} \omega \mathrm{d} \mathbf{v}_{*} \mathrm{~d} \mathbf{v} \\
& =\int_{\mathbb{R}^{3} \times \mathbb{R}^{3} \times \mathbb{S}^{2}} B_{K}\left(\mathbf{v}-\mathbf{v}_{*}, \omega\right)\left|f(\mathbf{v}) g\left(\mathbf{v}_{*}\right) h\left(\mathbf{v}^{\prime}\right)\right| \sqrt{1+|\mathbf{v}|^{2}+\left|\mathbf{v}_{*}\right|^{2}} \mathrm{~d} \omega \mathrm{d} \mathbf{v}_{*} \mathrm{~d} \mathbf{v} \\
& \leq 2 K\|f\|_{L_{1}^{1}}\|g\|_{L_{1}^{1}}\|h\|_{L^{1}} .
\end{aligned}
$$

To prove the main result of this section, we need two lemmas:

Lemma 3.2. Let $F \in \mathcal{B}_{2}^{+}\left(\mathbb{R}^{3}\right)$ and suppose

$$
\rho=\int_{\mathbb{R}^{3}} \mathrm{~d} F(\mathbf{v})>0, \quad \mathbf{u}=\frac{1}{\rho} \int_{\mathbb{R}^{3}} \mathbf{v} \mathrm{d} F(\mathbf{v}), \quad T=\frac{1}{3 \rho} \int_{\mathbb{R}^{3}}|\mathbf{v}-\mathbf{u}|^{2} \mathrm{~d} F(\mathbf{v})>0 .
$$

Let $S(F)$ be defined by (1.30),(1.31),(1.32), and let $0 \leq f \in L_{2}^{1}\left(\mathbb{R}^{3}\right)$ be the regular part of $F$. Then $S(F)=S(f)$. Moreover there is a sequence $0 \leq f_{n} \in L_{2}^{1}\left(\mathbb{R}^{3}\right)(n \in \mathbb{N})$ satisfying

$$
\int_{\mathbb{R}^{3}}\left(1, \mathbf{v},|\mathbf{v}|^{2} / 2\right) f_{n}(\mathbf{v}) \mathrm{d} \mathbf{v}=\int_{\mathbb{R}^{3}}\left(1, \mathbf{v},|\mathbf{v}|^{2} / 2\right) \mathrm{d} F(\mathbf{v}) \quad \forall n \in \mathbb{N}
$$

such that the weak convergence (1.32) holds and

$$
S\left(f_{n}\right) \geq\left(1-\frac{1}{2 n}\right) S(F) \quad \forall n \in \mathbb{N} .
$$

Consequently we have $\lim _{n \rightarrow \infty} S\left(f_{n}\right)=S(F)$. Furthermore, if $F$ is also isotropic, i.e. if $F$ is defined by a measure $\widetilde{F} \in \mathcal{B}_{1}^{+}\left(\mathbb{R}_{\geq 0}\right)$ through (1.14) or (1.16), then all $f_{n}$ can be also isotropic: $f_{n}(\mathbf{v})=\widetilde{f}_{n}\left(|\mathbf{v}|^{2} / 2\right)$, and thus $\widetilde{f}_{n}$ converges weakly to $\widetilde{F}$, i.e.

$$
\lim _{n \rightarrow \infty} \int_{\mathbb{R}_{\geq 0}} \varphi(x) \tilde{f}_{n}(x) \sqrt{x} \mathrm{~d} x=\int_{\mathbb{R}_{\geq 0}} \varphi(x) \mathrm{d} \widetilde{F}(x) \quad \forall \varphi \in C_{b}\left(\mathbb{R}_{\geq 0}\right) .
$$


Proof. Write $\mathrm{d} F(\mathbf{v})=f(\mathbf{v}) \mathrm{d} \mathbf{v}+\mathrm{d} \nu(\mathbf{v})$ with $0 \leq f \in L_{2}^{1}\left(\mathbb{R}^{3}\right)$ and $\nu \in \mathcal{B}_{2}^{+}\left(\mathbb{R}^{3}\right)$ is the singular part of $F$. We have proved in Lemma 3 of $[20]$ that $S(F) \leq S(f)$. The proof of the converse inequality $S(F) \geq S(f)$ is included in the proof of the second part of this lemma.

For every $n \in \mathbb{N}$, let $\mu_{n} \in \mathcal{B}_{2}^{+}\left(\mathbb{R}^{3}\right)$ be defined by $\mathrm{d} \mu_{n}(\mathbf{v})=\frac{1}{2 n} f(\mathbf{v})+\mathrm{d} \nu(\mathbf{v})$. From the assumption (3.7) it is easily seen that $\rho_{n}:=\int_{\mathbb{R}^{3}} \mathrm{~d} \mu_{n}(\mathbf{v})>0, T_{n}=\frac{1}{3 \rho_{n}} \int_{\mathbb{R}^{3}}\left|\mathbf{v}-\mathbf{u}_{n}\right|^{2} \mathrm{~d} \mu_{n}(\mathbf{v})>0$, where $\mathbf{u}_{n}=\frac{1}{\rho_{n}} \int_{\mathbb{R}^{3}} \mathbf{v} \mathrm{d} \mu_{n}(\mathbf{v})$. Also by considering $\int_{\mathbb{R}^{3}} \mathrm{~d} \nu(\mathbf{v})>0$ and $\int_{\mathbb{R}^{3}} \mathrm{~d} \nu(\mathbf{v})=0$ respectively it is easily seen that $\sup _{n \geq 1} \frac{1}{\rho_{n}} \int_{\mathbb{R}^{3}}|\mathbf{v}|^{2} \mathrm{~d} \mu_{n}(\mathbf{v})<\infty$ and thus

$$
\sup _{n \geq 1}\left\{\rho_{n},\left|\mathbf{u}_{n}\right|, T_{n}\right\}<\infty
$$

Let $0 \leq h_{n} \in L_{2}^{1}\left(\mathbb{R}^{3}\right)$ be the Mehler transform of $\mu_{n}$, i.e.

$$
h_{n}(\mathbf{v})=e^{3 n} \int_{\mathbb{R}^{3}} M_{1,0, T_{n}}\left(e^{n}\left(\mathbf{v}-\mathbf{u}_{n}-\sqrt{1-e^{-2 n}}\left(\mathbf{v}_{*}-\mathbf{u}_{n}\right)\right)\right) \mathrm{d} \mu_{n}\left(\mathbf{v}_{*}\right), \quad \mathbf{v} \in \mathbb{R}^{3}
$$

where $M_{1,0, T_{n}}(\mathbf{v})=\left(2 \pi T_{n}\right)^{-3 / 2} \exp \left(-\frac{|\mathbf{v}|^{2}}{2 T_{n}}\right)$. As is well-known, for any Borel measurable function $\psi$ on $\mathbb{R}^{3}$ satisfying $\sup _{\mathbf{v} \in \mathbb{R}^{3}}|\psi(\mathbf{v})|\left(1+|\mathbf{v}|^{2}\right)^{-1}<\infty$, we have (see e.g. [19])

$$
\begin{aligned}
& \int_{\mathbb{R}^{3}} \psi(\mathbf{v}) h_{n}(\mathbf{v}) \mathrm{d} \mathbf{v}=\int_{\mathbb{R}^{3}} M_{1,0,1}(\mathbf{v})\left(\int_{\mathbb{R}^{3}} \psi\left(e^{-n} T_{n}^{1 / 2} \mathbf{v}+\mathbf{u}_{n}+\sqrt{1-e^{-2 n}}\left(\mathbf{v}_{*}-\mathbf{u}_{n}\right)\right) \mathrm{d} \mu_{n}\left(\mathbf{v}_{*}\right)\right) \mathrm{d} \mathbf{v}, \\
& \int_{\mathbb{R}^{3}}\left(1, \mathbf{v},|\mathbf{v}|^{2} / 2\right) h_{n}(\mathbf{v}) \mathrm{d} \mathbf{v}=\int_{\mathbb{R}^{3}}\left(1, \mathbf{v},|\mathbf{v}|^{2} / 2\right) \mathrm{d} \mu_{n}(\mathbf{v}) \quad \forall n \in \mathbb{N} .
\end{aligned}
$$

Then together with (3.9) it is easily proved the weak convergence:

$$
\lim _{n \rightarrow \infty} \int_{\mathbb{R}^{3}} \psi(\mathbf{v}) h_{n}(\mathbf{v}) \mathrm{d} \mathbf{v}=\lim _{n \rightarrow \infty} \int_{\mathbb{R}^{3}} \psi(\mathbf{v}) \mathrm{d} \mu_{n}(\mathbf{v})=\int_{\mathbb{R}^{3}} \psi(\mathbf{v}) \mathrm{d} \nu(\mathbf{v}) \quad \forall \psi \in C_{b}\left(\mathbb{R}^{3}\right) .
$$

Let

$$
f_{n}(\mathbf{v})=\left(1-\frac{1}{2 n}\right) f(\mathbf{v})+h_{n}(\mathbf{v}) .
$$

Then $0 \leq f_{n} \in L_{2}^{1}\left(\mathbb{R}^{3}\right)$ have the same mass, momentum, and energy as $F$, and $f_{n}$ converge weakly to $F$ in the sense of (1.32). Using the entropy properties (1.28), (1.27) we have $S\left(f_{n}\right) \geq$ $\left(1-\frac{1}{2 n}\right) S(f)+\frac{1}{2 n} S\left(2 n h_{n}\right) \geq\left(1-\frac{1}{2 n}\right) S(f)$ for all $n \geq 1$. This together with lim $\sup S\left(f_{n}\right) \leq S(F)$ implies $S(f) \leq S(F)$ and thus we conclude $S(F)=S(f)$ and $\lim _{n \rightarrow \infty} S\left(f_{n}\right)=\stackrel{n \rightarrow \infty}{S}(F)$.

Finally suppose further that $F$ is defined by a measure $\stackrel{n}{F} \in \mathcal{B}_{1}^{+}\left(\mathbb{R}_{\geq 0}\right)$ through (1.14) or (1.16). Write $\mathrm{d} \widetilde{F}(x)=\widetilde{f}(x) \sqrt{x} \mathrm{~d} x+\mathrm{d} \widetilde{\nu}(x)$ where $0 \leq \widetilde{f} \in L^{1}\left(\mathbb{R}_{\geq 0},(1+x) \sqrt{x} \mathrm{~d} x\right)$ and $\widetilde{\nu}$ is the singular part of $\widetilde{F}$. Then, by uniqueness of the regular-singular decomposition, we have $f(\mathbf{v})=\widetilde{f}\left(|\mathbf{v}|^{2} / 2\right)$ (up to a null set) and $\nu$ is equal to the measure defined by $\widetilde{\nu}$ through (1.14) or equivalently (1.16). In this case we have $\mathbf{u}_{n}=\mathbf{0}$ and it is easily seen that $h_{n}(\mathbf{v})=: \widetilde{h}_{n}\left(|\mathbf{v}|^{2} / 2\right)$ are isotropic and thus $f_{n}(\mathbf{v})=\left(1-\frac{1}{2 n}\right) \widetilde{f}\left(|\mathbf{v}|^{2} / 2\right)+\widetilde{h}_{n}\left(|\mathbf{v}|^{2} / 2\right)=: \widetilde{f}_{n}\left(|\mathbf{v}|^{2} / 2\right)$ are isotropic. The weak convergence (3.8) follows from the weak convergence (1.32) of $f_{n}$ to $F$. 
Lemma 3.3. Let $f, g, h: \mathbb{R}_{\geq 0} \rightarrow \mathbb{R}_{\geq 0}$ be Lebesgue measurable and $0<a \leq b \wedge c$. Then

$$
\begin{aligned}
& \int_{\mathbb{R}^{3} \times \mathbb{R}^{3} \times \mathbb{S}^{2}}\left|\left(\mathbf{v}-\mathbf{v}_{*}\right) \cdot \omega\right| 1_{\{|\mathbf{v}| \leq a\}} 1_{\left\{\left|\mathbf{v}^{\prime}\right| \geq b\right\}} 1_{\left\{\left|\mathbf{v}_{*}^{\prime}\right| \geq c\right\}} f\left(|\mathbf{v}|^{2} / 2\right) g\left(\left|\mathbf{v}^{\prime}\right|^{2} / 2\right) h\left(\left|\mathbf{v}_{*}^{\prime}\right|^{2} / 2\right) \mathrm{d} \omega \mathrm{d} \mathbf{v}_{*} \mathrm{~d} \mathbf{v} \\
& =\left(\int_{|\mathbf{v}| \leq a} f\left(|\mathbf{v}|^{2} / 2\right) \mathrm{d} \mathbf{v}\right)\left(\int_{|\mathbf{v}| \geq b} \frac{1}{|\mathbf{v}|} g\left(|\mathbf{v}|^{2} / 2\right) \mathrm{d} \mathbf{v}\right)\left(\int_{|\mathbf{v}| \geq c} \frac{1}{|\mathbf{v}|} h\left(|\mathbf{v}|^{2} / 2\right) \mathrm{d} \mathbf{v}\right)
\end{aligned}
$$

Proof. Using the first equality in (6.10) (see Appendix) to $\Phi(r, \rho) \equiv(4 \pi)^{2}, \Psi(x, y, z)=$ $1_{\{\sqrt{2 x} \leq a\}} 1_{\{\sqrt{2 y} \geq b\}} 1_{\{\sqrt{2 z} \geq c\}} f(x) g(y) h(z)$ and using $(1.23)$ and noting that $\min \left\{\sqrt{x}, \sqrt{x_{*}}, \sqrt{y}, \sqrt{z}\right\}=$ $\sqrt{x}$ for $0 \leq x \leq y \wedge z$, we have

$$
\begin{aligned}
& \text { the 1.h.s. of }(3.10)=\sqrt{2}(4 \pi)^{3} \int_{\mathbb{R}_{\geq 0}^{3}} \sqrt{x} 1_{\{\sqrt{2 x} \leq a\}} 1_{\{\sqrt{2 y} \geq b\}} 1_{\{\sqrt{2 z} \geq c\}} f(x) g(y) h(z) \mathrm{d} x \mathrm{~d} y \mathrm{~d} z \\
& =\text { the r.h.s. of }(3.10) .
\end{aligned}
$$

To prove the main result Proposition 3.4, we will also use the following inequalities: if $0 \leq f \in L_{2}^{1}\left(\mathbb{R}^{3}\right)$, then

$$
\operatorname{mes}\left(\left\{\mathbf{v} \in \mathbb{R}^{3} \mid f(\mathbf{v})>1 / 3\right\}\right) \leq 2 S(f), \quad \int_{f(\mathbf{v})<9} f(\mathbf{v}) \mathrm{d} \mathbf{v} \leq 5 S(f) .
$$

In fact for the function $s(y)=(1+y) \log (1+y)-y \log y$ we have $s(y) \geq 2 \frac{y}{1+y}, y \geq 0$. So $1_{\{f(\mathbf{v})>1 / 3\}} s(f(\mathbf{v})) \geq \frac{1}{2} 1_{\{f(\mathbf{v})>1 / 3\}}, 1_{\{f(\mathbf{v})<9\}} s(f(\mathbf{v})) \geq \frac{1}{5} 1_{\{f(\mathbf{v})<9\}} f(\mathbf{v})$, hence (3.11) follows.

Proposition 3.4. Let $B\left(\mathbf{v}-\mathbf{v}_{*}, \omega\right)$ satisfy Assumption 1.1. Given any $N>0, E>0$.

(I) Let $B_{K}\left(\mathbf{v}-\mathbf{v}_{*}, \omega\right)$ be given by (3.6) (or equivalently (3.21),(3.22)), let $\left\{f_{0}^{K}=f_{0}^{K}\left(|\mathbf{v}|^{2} / 2\right)\right\}_{K \in \mathbb{N}}$ be any sequence of nonnegative isotropic functions in $L_{2}^{1}\left(\mathbb{R}^{3}\right)$ satisfying

$$
\int_{\mathbb{R}^{3}}\left(1,|\mathbf{v}|^{2} / 2\right) f_{0}^{K}\left(|\mathbf{v}|^{2} / 2\right) \mathrm{d} \mathbf{v}=4 \pi \sqrt{2}(N, E) \quad \forall K \in \mathbb{N} .
$$

Then for every $K \in \mathbb{N}$, there exists a unique conservative isotropic approximate solution $f^{K}=$ $f^{K}\left(|\mathbf{v}|^{2} / 2, t\right)$ of Eq.(1.1) on $\mathbb{R}^{3} \times[0, \infty)$ corresponding to the approximate kernel $B_{K}$ such that $\left.f^{K}\right|_{t=0}=f_{0}^{K}$, and it holds the moment production:

$$
\sup _{K \in \mathbb{N}}\left\|f^{K}(t)\right\|_{L_{s}^{1}} \leq C_{s}(1+1 / t)^{s-2} \quad \forall t>0, \forall s>2
$$

where the constant $0<C_{s}<\infty$ depends only on $N, E, b_{0}, \eta$ and $s$.

Moreover for any $t_{0}>0$, let

$$
S_{*}\left(t_{0}\right)=\min \left\{\frac{7 \pi a^{3}}{24}, \frac{4 \pi^{2} E^{2}}{5 C\left(1+2 / t_{0}\right)^{2}}, b_{0} \min \left\{1,\left(2 a^{2}\right)^{1 \vee \eta}\right\} \frac{7 \pi^{4} \sqrt{2} a^{3} E^{5} t_{0}}{96 C^{3}\left(1+2 / t_{0}\right)^{6}}\right\}
$$


where $a=\frac{1}{2} \sqrt{E / N}, 0<C=C_{4}<\infty$ is the constant in (3.13) for $s=4$ so that $C$ depends only on $N, E, b_{0}, \eta$. Then

$$
S\left(f^{K}(t)\right) \geq S\left(f^{K}\left(t_{0}\right)\right) \geq S_{*}\left(t_{0}\right) \quad \forall t \geq t_{0}, \forall K \in \mathbb{N} .
$$

(II) Let $F_{0} \in \mathcal{B}_{1}^{+}\left(\mathbb{R}_{\geq 0}\right)$ satisfy $N\left(F_{0}\right)=N, E\left(F_{0}\right)=E$. Then there exists a conservative measure-valued isotropic solution $F_{t}$ of Eq.(1.1) on $[0, \infty)$ with the initial datum $F_{0}$, such that

$$
S\left(F_{t}\right) \geq S_{*}\left(t_{0}\right) \quad \forall t \geq t_{0}
$$

for all $t_{0}>0$, and

$$
M_{p}\left(F_{t}\right) \leq C_{p}(1+1 / t)^{2 p-2} \quad \forall t>0, \forall p>1
$$

where the constant $0<C_{p}<\infty$ depends only on $N, E, b_{0}, \eta$ and $p$.

Moreover there exists a sequence $\left\{f^{K}\right\}_{K \in \mathbb{N}}$ of a conservative isotropic approximate solutions of Eq.(1.1) on $\mathbb{R}^{3} \times[0, \infty)$ presented in part (I) such that their initial data sequence $\left\{f_{0}^{K}\right\}_{K \in \mathbb{N}}$ satisfies

$$
\lim _{K \rightarrow \infty} S\left(f_{0}^{K}\right)=S\left(F_{0}\right)
$$

and there is a subsequence $\left\{f^{K_{n}}\right\}_{n \in \mathbb{N}}$ such that $F_{t}$ and the measure $\bar{F}_{t}$ defined by $F_{t}$ through (1.14) or (1.16) are weak limits of $f^{K_{n}}(x, t)$ and $f^{K_{n}}\left(|\mathbf{v}|^{2} / 2, t\right)$ respectively, i.e.

$$
\begin{array}{cl}
\int_{\mathbb{R}_{\geq 0}} \varphi(x) \mathrm{d} F_{t}(x)=\lim _{n \rightarrow \infty} \int_{\mathbb{R}_{\geq 0}} \varphi(x) f^{K_{n}}(x, t) \sqrt{x} \mathrm{~d} x \quad & \forall \varphi \in C_{b}\left(\mathbb{R}_{\geq 0}\right), \forall t \geq 0 \\
\int_{\mathbb{R}^{3}} \psi(\mathbf{v}) \mathrm{d} \bar{F}_{t}(\mathbf{v})=\lim _{n \rightarrow \infty} \int_{\mathbb{R}^{3}} \psi(\mathbf{v}) f^{K_{n}}\left(|\mathbf{v}|^{2} / 2, t\right) \mathrm{d} \mathbf{v} \quad & \forall \psi \in C_{b}\left(\mathbb{R}^{3}\right), \quad \forall t \geq 0 .
\end{array}
$$

Proof. (I): Let us rewrite (3.6) as

$$
\begin{aligned}
& B_{K}\left(\mathbf{v},-\mathbf{v}_{*}, \omega\right)=\frac{\left|\left(\mathbf{v}-\mathbf{v}_{*}\right) \cdot \omega\right|}{(4 \pi)^{2}} \Phi_{K}\left(\left|\mathbf{v}-\mathbf{v}^{\prime}\right|,\left|\mathbf{v}-\mathbf{v}_{*}^{\prime}\right|\right), \\
& \text { with } \Phi_{K}(r, \rho)=\min \left\{\Phi(r, \rho),(4 \pi)^{2} K r \rho\right\} .
\end{aligned}
$$

The existence, uniqueness and the uniform moment production (3.13) of the conservative isotropic approximate solutions $f^{K}$ have been essentially proven in the proofs of Theorem 2, Theorem 3 in [18] and Theorem 4 in [19]. The only difference from the present case is that [18], [19] consider those approximate kernels $B_{K}\left(\mathbf{v}-\mathbf{v}_{*}, \omega\right)$ in (3.6) where $B\left(\mathbf{v}-\mathbf{v}_{*}, \omega\right)=$ $b(\cos (\theta))\left|\mathbf{v}-\mathbf{v}_{*}\right|^{\gamma}\left(\right.$ with $\theta=\arccos \left(\left|\left(\mathbf{v}-\mathbf{v}_{*}\right) \cdot \omega\right| /\left|\mathbf{v}-\mathbf{v}_{*}\right|\right)$ with $0<\gamma \leq 1$ and $0 \leq b(\cdot) \in$ $C([0,1]), b(0)=0, \int_{0}^{1} b(\tau) \mathrm{d} \tau>0$. However, for the present case, one sees from (1.11) that for the case $\left|\mathbf{v}-\mathbf{v}_{*}\right| \geq 1$, the the lower bound $\frac{b_{0}}{(4 \pi)^{2}} \cos (\theta)\left|\mathbf{v}-\mathbf{v}_{*}\right|$ and the upper bound $\frac{1}{(4 \pi)^{2}} \cos (\theta)\left|\mathbf{v}-\mathbf{v}_{*}\right|$ of $B\left(\mathbf{v}-\mathbf{v}_{*}, \omega\right)$ have the same form as used in [19] with $\gamma=1$, and thus the proof of (3.13) is completely the same as the proof of Theorem 4 in [19] (and thus the proof 
of the uniqueness of $f^{K}$ is also valid for the present kernel $B_{K}$.) In fact to see this is really the case, one needs only to prove the following inequality

$$
\int_{\mathbb{R}^{3} \times \mathbb{R}^{3} \times \mathbb{S}^{2}} B_{K}\left(\mathbf{v}-\mathbf{v}_{*}, \omega\right) f f_{*}[\kappa(\theta)]^{s}|\mathbf{v}|^{s} \mathrm{~d} \omega \mathrm{d} \mathbf{v} \mathrm{d} \mathbf{v}_{*} \geq \frac{A_{s}}{2}\left(\|f\|_{L^{1}} M_{s+1}(f)-\frac{1}{4}\left(\|f\|_{L^{1}}\right)^{2}\right)
$$

where

$$
A_{s}=4 \pi \int_{0}^{\pi / 2} \min \left\{\frac{b_{0}}{(4 \pi)^{2}}, \cos (\theta) \sin (\theta)\right\} \sin (\theta) \cos (\theta)[\kappa(\theta)]^{s} \mathrm{~d} \theta,
$$

$\kappa(\theta)=\min \{\cos (\theta), 1-\cos (\theta)\}$, and $M_{s}(f)=\int_{\mathbb{R}^{3}}|\mathbf{v}|^{s} f(\mathbf{v}) \mathrm{d} \mathbf{v}$. To do this we first note that from (3.6) and Assumption 1.1 we have

$$
B_{K}\left(\mathbf{v}-\mathbf{v}_{*}, \omega\right) \geq\left|\mathbf{v}-\mathbf{v}_{*}\right| \cos (\theta) \min \left\{\frac{b_{0}}{(4 \pi)^{2}}, \cos (\theta) \sin (\theta)\right\} \min \left\{1,\left|\mathbf{v}-\mathbf{v}_{*}\right|^{2 \vee 2 \eta}\right\} .
$$

Then for all $s>0,0 \leq f(\mathbf{v})=f\left(|\mathbf{v}|^{2} / 2\right) \in L_{s+1}^{1}\left(\mathbb{R}^{3}\right)$, we have

$$
\begin{aligned}
& \int_{\mathbb{R}^{3} \times \mathbb{R}^{3} \times \mathbb{S}^{2}} B_{K}\left(\mathbf{v}-\mathbf{v}_{*}, \omega\right) f f_{*}[\kappa(\theta)]^{s}|\mathbf{v}|^{s} \mathrm{~d} \omega \mathrm{d} \mathbf{v} \mathrm{d} \mathbf{v}_{*} \\
& \geq A_{s} \int_{\mathbb{R}^{3} \times \mathbb{R}^{3}} f f_{*}|\mathbf{v}|^{s}\left|\mathbf{v}-\mathbf{v}_{*}\right| \min \left\{1,\left|\mathbf{v}-\mathbf{v}_{*}\right|^{2 \vee} 2 \eta\right\} \mathrm{d} \mathbf{v} \mathrm{d} \mathbf{v}_{*}
\end{aligned}
$$

To estimate the right-hand side of (3.24), we will use the following inequality (the proof is esiy): if $\Psi$ is nonnegative Lebesgue measurable on $\mathbb{R}_{\geq 0}^{3}$ satisfying that $\rho \mapsto \Psi\left(r, r_{*}, \rho\right)$ is non-decreasing on $[0, \infty)$ for all $r, r_{*} \geq 0$, then (using spherical coordinates transform)

$$
\int_{\mathbb{R}^{3} \times \mathbb{R}^{3}} \Psi\left(|\mathbf{v}|,\left|\mathbf{v}_{*}\right|,\left|\mathbf{v}-\mathbf{v}_{*}\right|\right) \mathrm{d} \mathbf{v} \mathrm{d} \mathbf{v}_{*} \geq \frac{1}{2} \int_{\mathbb{R}^{3} \times \mathbb{R}^{3}} \Psi\left(|\mathbf{v}|,\left|\mathbf{v}_{*}\right|,|\mathbf{v}|\right) \mathrm{d} \mathbf{v} \mathrm{d} \mathbf{v}_{*} .
$$

Using this inequality and noting that $\min \left\{1,|\mathbf{v}|^{2 \vee 2 \eta}\right\}=1-\left(1-|\mathbf{v}|^{2 \vee 2 \eta}\right)+$ we obtain (3.23):

$$
\begin{aligned}
& \int_{\mathbb{R}^{3} \times \mathbb{R}^{3}} f f_{*}|\mathbf{v}|^{s}\left|\mathbf{v}-\mathbf{v}_{*}\right| \min \left\{1,\left|\mathbf{v}-\mathbf{v}_{*}\right|^{2}\right\} \mathrm{d} \mathbf{v} \mathrm{d} \mathbf{v}_{*} \\
& \geq \frac{1}{2} \int_{\mathbb{R}^{3} \times \mathbb{R}^{3}} f f_{*}|\mathbf{v}|^{s+1} \mid \min \left\{1,|\mathbf{v}|^{2}\right\} \mathrm{d} \mathbf{v} \mathrm{d} \mathbf{v}_{*} \geq \frac{1}{2}\left(\|f\|_{L^{1}} M_{s+1}(f)-\frac{1}{4}\left(\|f\|_{L^{1}}\right)^{2}\right) .
\end{aligned}
$$

Now we are going to prove the positive lower bound (3.15) of entropy, which is the new thing of the proposition. Given any $t_{0}>0, K \in \mathbb{N}$. For notation convenience we denote

$$
f\left(|\mathbf{v}|^{2} / 2, t\right):=f^{K}\left(|\mathbf{v}|^{2} / 2, t\right)
$$

Since $t \mapsto S(f(t))$ is non-decreasing, we need only prove that $S\left(f\left(t_{0}\right)\right) \geq S_{*}\left(t_{0}\right)$. To do this we may assume that (recall $S_{*}\left(t_{0}\right)$ in $\left.(3.14)\right)$

$$
S\left(f\left(t_{0}\right)\right) \leq \min \left\{\frac{7 \pi a^{3}}{24}, \frac{4 \pi^{2} E^{2}}{5 C\left(1+2 / t_{0}\right)^{2}}\right\} .
$$


Recall $a=\frac{1}{2} \sqrt{E / N}$ and let

$$
b=\left(\frac{C\left(1+2 / t_{0}\right)^{2}}{2 \pi \sqrt{2} E}\right)^{1 / 2} .
$$

By conservation of mass and energy and moment production (3.13) we have

$$
\begin{aligned}
& \int_{|\mathbf{v}| \leq 2 a} \frac{1}{2}|\mathbf{v}|^{2} f\left(|\mathbf{v}|^{2} / 2, t\right) \mathrm{d} \mathbf{v} \leq \frac{4 a^{2}}{2} 4 \pi \sqrt{2} N=2 \pi \sqrt{2} E, \\
& \int_{|\mathbf{v}|>2 a} \frac{1}{2}|\mathbf{v}|^{2} f\left(|\mathbf{v}|^{2} / 2, t\right) \mathrm{d} \mathbf{v} \geq 2 \pi \sqrt{2} E \\
& \int_{\mathbb{R}^{3}}|\mathbf{v}|^{4} f\left(|\mathbf{v}|^{2} / 2, t\right) \mathrm{d} \mathbf{v} \leq C\left(1+2 / t_{0}\right)^{2} \quad \forall t \geq t_{0} / 2 .
\end{aligned}
$$

Since, by Cauchy-Schwarz inequality, $E^{2} / N \leq \frac{1}{16 \pi \sqrt{2}} \int_{\mathbb{R}^{3}}|\mathbf{v}|^{4} f\left(|\mathbf{v}|^{2} / 2, t\right) \mathrm{d} \mathbf{v}$, it follows that $b \geq$ $4 \sqrt{2} a>2 a$. Also since for all $t \geq t_{0} / 2$

$$
\int_{|\mathbf{v}| \geq b} \frac{1}{2}|\mathbf{v}|^{2} f \mathrm{~d} \mathbf{v} \leq \frac{1}{2 b^{2}} \int_{\mathbb{R}^{3}}|\mathbf{v}|^{4} f \mathrm{~d} \mathbf{v} \leq \frac{1}{2 b^{2}} C\left(1+2 / t_{0}\right)^{2}=\pi \sqrt{2} E
$$

it follows that

$$
\begin{aligned}
& \int_{2 a \leq \mathbf{v} \mid \leq b} f\left(|\mathbf{v}|^{2} / 2, t\right) \mathrm{d} \mathbf{v} \geq \frac{2}{b^{2}}\left(\int_{|\mathbf{v}| \geq 2 a} \frac{1}{2}|\mathbf{v}|^{2} f \mathrm{~d} \mathbf{v}-\int_{|\mathbf{v}| \geq b} \frac{1}{2}|\mathbf{v}|^{2} f \mathrm{~d} \mathbf{v}\right) \\
& \geq \frac{2}{b^{2}}(2 \pi \sqrt{2} E-\pi \sqrt{2} E)=\frac{8 \pi^{2} E^{2}}{C\left(1+2 / t_{0}\right)^{2}} .
\end{aligned}
$$

Let

$$
\begin{aligned}
& \mathcal{V}_{t}=\left\{\left(\mathbf{v}, \mathbf{v}_{*}, \omega\right) \in \mathbb{R}^{3} \times \mathbb{R}^{3} \times \mathbb{S}^{2}|a / 2 \leq| \mathbf{v}|\leq a, 2 a \leq| \mathbf{v}^{\prime}|\leq b, 2 a \leq| \mathbf{v}_{*}^{\prime} \mid \leq b,\right. \\
& \left.f\left(|\mathbf{v}|^{2} / 2, t\right) \leq 1 / 3, f\left(\left|\mathbf{v}^{\prime}\right|^{2} / 2, t\right) \geq 9, f\left(\left|\mathbf{v}_{*}^{\prime}\right|^{2} / 2, t\right) \geq 9\right\}, \quad t \geq t_{0} / 2 .
\end{aligned}
$$

Then for all $\left(\mathbf{v}, \mathbf{v}_{*}, \omega\right) \in \mathcal{V}_{t}$ we have: $\left|\mathbf{v}-\mathbf{v}^{\prime}\right| \geq a,\left|\mathbf{v}-\mathbf{v}_{*}^{\prime}\right| \geq a$ and so

$$
\begin{aligned}
& B_{K}\left(\mathbf{v}-\mathbf{v}_{*}, \omega\right) \geq \frac{\left|\left(\mathbf{v}-\mathbf{v}_{*}\right) \cdot \omega\right|}{(4 \pi)^{2}} \min \left\{b_{0} \min \left\{1,\left(2 a^{2}\right)^{\eta}\right\},(4 \pi)^{2} a^{2}\right\} \\
& \geq \frac{\left|\left(\mathbf{v}-\mathbf{v}_{*}\right) \cdot \omega\right|}{(4 \pi)^{2}} b_{0} \min \left\{1,\left(2 a^{2}\right)^{1 \vee \eta}\right\}, \\
& \Pi(f) \geq f\left(\left|\mathbf{v}^{\prime}\right|^{2} / 2, t\right) f\left(\left|\mathbf{v}_{*}^{\prime}\right|^{2} / 2, t\right) \geq \frac{1}{b^{2}}\left|\mathbf{v}^{\prime}\right|\left|\mathbf{v}_{*}^{\prime}\right| f\left(\left|\mathbf{v}^{\prime}\right|^{2} / 2, t\right) f\left(\left|\mathbf{v}_{*}^{\prime}\right|^{2} / 2, t\right),
\end{aligned}
$$

and, for $g(\cdot, t):=f(\cdot, t) /(1+f(\cdot, t))(\leq 1)$,

$$
\begin{gathered}
g=g\left(|\mathbf{v}|^{2} / 2, t\right) \leq 1 / 4, \quad g^{\prime}=g\left(\left|\mathbf{v}^{\prime}\right|^{2} / 2, t\right) \geq 9 / 10, \quad g_{*}^{\prime}=g\left(\left|\mathbf{v}_{*}^{\prime}\right|^{2} / 2, t\right) \geq 9 / 10, \\
\Gamma\left(g^{\prime} g_{*}^{\prime}, g g_{*}\right)=\left(g^{\prime} g_{*}^{\prime}-g g_{*}\right) \log \left(\frac{g^{\prime} g_{*}^{\prime}}{g g_{*}}\right) \geq\left((9 / 10)^{2}-1 / 4\right) \log \left((9 / 10)^{2} 4\right)>\frac{1}{2} .
\end{gathered}
$$


Thus

$$
\Pi(f) \Gamma\left(g^{\prime} g_{*}^{\prime}, g g_{*}\right) \geq \frac{1}{2 b^{2}}\left|\mathbf{v}^{\prime}\right| f\left(\left|\mathbf{v}^{\prime}\right|^{2} / 2, t\right)\left|\mathbf{v}_{*}^{\prime}\right| f\left(\left|\mathbf{v}_{*}^{\prime}\right|^{2} / 2, t\right)
$$

and so for all $t \geq t_{0} / 2$

$$
\begin{aligned}
& D_{K}(f(t)) \geq \frac{1}{4} \int_{\mathcal{V}_{t}} B_{K}\left(\mathbf{v}-\mathbf{v}_{*}, \omega\right) \Pi(f) \Gamma\left(g^{\prime} g_{*}^{\prime}, g g_{*}\right) \mathrm{d} \omega \mathrm{d} \mathbf{v}_{*} \mathrm{~d} \mathbf{v} \\
& \geq \frac{b_{0} \min \left\{1,\left(2 a^{2}\right)^{1 \vee \eta}\right\}}{8(4 \pi)^{2} b^{2}} \int_{\mathcal{V}_{t}}\left|\left(\mathbf{v}-\mathbf{v}_{*}\right) \cdot \omega\right|\left|\mathbf{v}^{\prime}\right| f\left(\left|\mathbf{v}^{\prime}\right|^{2} / 2, t\right)\left|\mathbf{v}_{*}^{\prime}\right| f\left(\left|\mathbf{v}_{*}^{\prime}\right|^{2} / 2, t\right) \mathrm{d} \omega \mathrm{d} \mathbf{v}_{*} \mathrm{~d} \mathbf{v} .
\end{aligned}
$$

We then compute using the formula (3.10) that

$$
\begin{aligned}
& \int_{\mathcal{V}_{t}}\left|\left(\mathbf{v}-\mathbf{v}_{*}\right) \cdot \omega\right|\left|\mathbf{v}^{\prime}\right| f\left(\left|\mathbf{v}^{\prime}\right|^{2} / 2, t\right)\left|\mathbf{v}_{*}^{\prime}\right| f\left(\left|\mathbf{v}_{*}^{\prime}\right|^{2} / 2, t\right) \mathrm{d} \omega \mathrm{d} \mathbf{v}_{*} \mathrm{~d} \mathbf{v} \\
& =\int_{\mathbb{R}^{3} \times \mathbb{R}^{3} \times \mathbb{S}^{2}}\left|\left(\mathbf{v}-\mathbf{v}_{*}\right) \cdot \omega\right| 1_{\{a / 2 \leq|\mathbf{v}| \leq a\}} 1_{\left\{f\left(|\mathbf{v}|^{2} / 2, t\right) \leq 1 / 3\right\}} 1_{\left\{2 a \leq\left|\mathbf{v}^{\prime}\right| \leq b\right\}} 1_{\left\{2 a \leq\left|\mathbf{v}_{*}^{\prime}\right| \leq b\right\}} \\
& \times 1_{\left\{f\left(\left|\mathbf{v}^{\prime}\right|^{2} / 2, t\right) \geq 9\right\}} 1_{\left\{f\left(\left|\mathbf{v}_{*}^{\prime}\right|^{2} / 2, t\right) \geq 9\right\}}\left|\mathbf{v}^{\prime}\right| f\left(\left|\mathbf{v}^{\prime}\right|^{2} / 2, t\right)\left|\mathbf{v}_{*}^{\prime}\right| f\left(\left|\mathbf{v}_{*}^{\prime}\right|^{2} / 2, t\right) \mathrm{d} \omega \mathrm{d} \mathbf{v}_{*} \mathrm{~d} \mathbf{v} \\
& =\operatorname{mes}\left(\left\{\mathbf{v} \in \mathbb{R}^{3}|a / 2 \leq| \mathbf{v} \mid \leq a, f\left(|\mathbf{v}|^{2} / 2, t\right) \leq 1 / 3\right\}\right)\left(\int_{2 a \leq|\mathbf{v}| \leq b, f\left(|\mathbf{v}|^{2} / 2, t\right) \geq 9} f\left(|\mathbf{v}|^{2} / 2, t\right) \mathrm{d} \mathbf{v}\right)^{2} .
\end{aligned}
$$

Also using (3.11) and the non-decrease of the entropy we have for all $t \in\left[t_{0} / 2, t_{0}\right]$

$$
\begin{aligned}
& \operatorname{mes}\left(\left\{\mathbf{v} \in \mathbb{R}^{3} \mid f\left(|\mathbf{v}|^{2} / 2, t\right)>1 / 3\right\}\right) \leq 2 S(f(t)) \leq 2 S\left(f\left(t_{0}\right)\right), \\
& \int_{f\left(|\mathbf{v}|^{2} / 2, t\right)<9} f\left(|\mathbf{v}|^{2} / 2, t\right) \mathrm{d} \mathbf{v} \leq 5 S(f(t)) \leq 5 S\left(f\left(t_{0}\right)\right)
\end{aligned}
$$

and so, using (3.25),(3.26),

$$
\begin{aligned}
& \operatorname{mes}\left(\left\{\mathbf{v} \in \mathbb{R}^{3}|a / 2 \leq| \mathbf{v} \mid \leq a, f\left(|\mathbf{v}|^{2} / 2, t\right) \leq 1 / 3\right\}\right) \\
& \geq \operatorname{mes}\left(\left\{\mathbf{v} \in \mathbb{R}^{3}|a / 2 \leq| \mathbf{v} \mid \leq a\right\}\right)-\operatorname{mes}\left(\left\{\mathbf{v} \in \mathbb{R}^{3} \mid f\left(|\mathbf{v}|^{2} / 2, t\right)>1 / 3\right\}\right) \\
& \geq \frac{7 \pi}{6} a^{3}-2 S\left(f\left(t_{0}\right)\right) \geq \frac{7 \pi}{12} a^{3}, \\
& \int_{2 a \leq|\mathbf{v}| \leq b, f\left(|\mathbf{v}|^{2} / 2, t\right) \geq 9} f\left(|\mathbf{v}|^{2} / 2, t\right) \mathrm{d} \mathbf{v} \geq \int_{2 a \leq|\mathbf{v}| \leq b} f \mathrm{~d} \mathbf{v}-\int_{f\left(|\mathbf{v}|^{2} / 2, t\right)<9} f \mathrm{~d} \mathbf{v} \\
& \geq \frac{8 \pi^{2} E^{2}}{C_{4}\left(1+2 / t_{0}\right)^{2}}-5 S\left(f\left(t_{0}\right)\right) \geq \frac{4 \pi^{2} E^{2}}{C_{4}\left(1+2 / t_{0}\right)^{2}} .
\end{aligned}
$$

Thus for all $t \in\left[t_{0} / 2, t_{0}\right]$ we have

$$
\int_{\mathcal{V}_{t}}\left|\left(\mathbf{v}-\mathbf{v}_{*}\right) \cdot \omega\right|\left|\mathbf{v}^{\prime}\right| f\left(|\mathbf{v}|^{2} / 2, t\right)\left|\mathbf{v}_{*}^{\prime}\right| f\left(\left|\mathbf{v}_{*}\right|^{2} / 2, t\right) \mathrm{d} \omega \mathrm{d} \mathbf{v}_{*} \mathrm{~d} \mathbf{v} \geq \frac{7 \pi a^{3}}{12}\left(\frac{4 \pi^{2} E^{2}}{C_{4}\left(1+2 / t_{0}\right)^{2}}\right)^{2}
$$


and so

$$
\begin{aligned}
& D_{K}(f(t)) \geq \frac{b_{0} \min \left\{1,\left(2 a^{2}\right)^{1 \vee \eta}\right\}}{8(4 \pi)^{2} b^{2}} \frac{7 \pi a^{3}}{12}\left(\frac{4 \pi^{2} E^{2}}{C\left(1+2 / t_{0}\right)^{2}}\right)^{2} \\
& =b_{0} \min \left\{1,\left(2 a^{2}\right)^{1 \vee \eta}\right\} \frac{7 \pi^{4} \sqrt{2} a^{3} E^{5}}{48 C^{3}\left(1+2 / t_{0}\right)^{6}}, \\
& S\left(f\left(t_{0}\right)\right)=S\left(f\left(t_{0} / 2\right)\right)+\int_{t_{0} / 2}^{t_{0}} D_{K}(f(t)) \mathrm{d} t \geq \int_{t_{0} / 2}^{t_{0}} D_{K}(f(t)) \mathrm{d} t \\
& \geq b_{0} \min \left\{1,\left(2 a^{2}\right)^{1 \vee \eta}\right\} \frac{7 \pi^{4} \sqrt{2} a^{3} E^{5}}{48 C^{3}\left(1+2 / t_{0}\right)^{6}} \frac{t_{0}}{2} \geq S_{*}\left(t_{0}\right) .
\end{aligned}
$$

This proves $(3.15)$.

(II): Let $\bar{F}_{0} \in B_{2}^{+}\left(\mathbb{R}^{3}\right)$ be the isotropic measure defined by $F_{0}$ through (1.14) or (1.16). For each $K \in \mathbb{N}$, let $0 \leq f_{0}^{K}=f_{0}^{K}\left(|\mathbf{v}|^{2} / 2\right) \in L_{2}^{1}\left(\mathbb{R}^{3}\right)$ be obtained in Lemma 3.2 for $\bar{F}_{0}$, so that $f_{0}^{K}$ satisfy $(3.12),(3.15)$ (because $\left.S\left(\bar{F}_{0}\right)=S\left(F_{0}\right)\right)$ and $(3.8)$ with $\widetilde{f}_{K}(x)=f_{0}^{K}(x), \widetilde{F}=F$. Let $f^{K}=f^{K}\left(|\mathbf{v}|^{2} / 2, t\right)$ be the conservative isotropic approximate solution of Eq.(1.1) on $\mathbb{R}^{3} \times[0, \infty)$ corresponding to the approximate kernel $B_{K}$ obtained in part (I) of the proposition satisfying $\left.f^{K}\right|_{t=0}=f_{0}^{K}$. Let $F_{t}^{K}, F_{0}^{K} \in \mathcal{B}_{1}^{+}\left(\mathbb{R}_{\geq 0}\right)$ be defined by $\mathrm{d} F_{t}^{K}(x)=f^{K}(x, t) \sqrt{x} \mathrm{~d} x, \mathrm{~d} F_{0}^{K}(x)=$ $f_{0}^{K}(x) \sqrt{x} \mathrm{~d} x$, then the measure $F_{t}^{K}$ with the initial datum $F_{0}^{K}$ is a conservative measure-valued isotropic solution of Eq.(1.1) on [0, $\infty$ ) in the sense of Definition 1.2 corresponding to the collision kernel $B_{K}\left(\mathbf{v}-\mathbf{v}_{*}, \omega\right)$ given by (3.21),(3.22). Since Definition 1.2 is equivalent to Definition 6.6 (see Appendix), it follows from Theorem 1 (Weak Stability) in [19] that there exist a conservative measure-valued isotropic solution $F_{t}$ of Eq.(1.1) on $[0, \infty)$ (corresponding to the kernel $B$ ) with the initial datum $F_{0}$, and a subsequence $\left\{f^{K_{n}}\right\}_{n \in \mathbb{N}}$, such that $F_{t}$ is the weak limit of $f^{K_{n}}(\cdot, t)$, i.e. (3.19) holds true. This implies that the weak convergence (3.20) also holds true. Thus by definition of entropy of measures and (3.15) we obtain

$$
S\left(F_{t}\right)=S\left(\bar{F}_{t}\right) \geq \limsup _{n \rightarrow \infty} S\left(f^{K_{n}}(t)\right) \geq S_{*}\left(t_{0}\right) \quad \forall t \geq t_{0}
$$

for all $t_{0}>0$. Finally since $M_{p}\left(F_{t}\right)=(4 \pi \sqrt{2})^{-1} \int_{\mathbb{R}^{3}}\left(|\mathbf{v}|^{2} / 2\right)^{p} \mathrm{~d} \bar{F}_{t}(\mathbf{v})$, the moment production (3.17) follows easily from the weak convergence (3.20) and (3.13).

\section{Rate of Entropy Convergence}

In this section we prove the first part of Theorem 1.4: algebraic rate of entropy convergence for measure-valued solutions. As usual, we start to work with approximate solutions then take weak limit to passing the result to a true solution. 
For convenience of proof and in order to connect some known results as mentioned in Section 2 for $\mathcal{D}_{2}(f)$, we will also use the $\sigma$-representation of $\left(\mathbf{v}^{\prime}, \mathbf{v}_{*}^{\prime}\right)$ :

$$
\mathbf{v}^{\prime}=\frac{\mathbf{v}+\mathbf{v}_{*}}{2}+\frac{\left|\mathbf{v}-\mathbf{v}_{*}\right|}{2} \sigma, \quad \mathbf{v}_{*}^{\prime}=\frac{\mathbf{v}+\mathbf{v}_{*}}{2}-\frac{\left|\mathbf{v}-\mathbf{v}_{*}\right|}{2} \sigma, \quad \sigma \in \mathbb{S}^{2}, \quad \mathbf{v}, \mathbf{v}_{*} \in \mathbb{R}^{3} .
$$

It is not difficult to deduce the following relation between the $\omega$-representation (1.2) and the $\sigma$-representation (4.1) (see e.g. Section 4 of Chapter 1 in [33]):

$$
\left.\int_{\mathbb{S}^{2}} \Psi\left(\mathbf{v}^{\prime}, \mathbf{v}_{*}^{\prime}\right)\right|_{\omega-\text { rep. }} \mathrm{d} \omega=\left.\int_{\mathbb{S}^{2}} \frac{\left|\mathbf{v}-\mathbf{v}_{*}\right|}{2\left|\mathbf{v}-\mathbf{v}^{\prime}\right|} \Psi\left(\mathbf{v}^{\prime}, \mathbf{v}_{*}^{\prime}\right)\right|_{\sigma-\text { rep. }} \mathrm{d} \sigma .
$$

In particular we have

$$
\left.\int_{\mathbb{S}^{2}} B_{K}\left(\mathbf{v}-\mathbf{v}_{*}, \omega\right) \Psi\left(\mathbf{v}^{\prime}, \mathbf{v}_{*}^{\prime}\right)\right|_{\omega-\text { rep. }} \mathrm{d} \omega=\left.\int_{\mathbb{S}^{2}} \bar{B}_{K}\left(\mathbf{v}-\mathbf{v}_{*}, \sigma\right) \Psi\left(\mathbf{v}^{\prime}, \mathbf{v}_{*}^{\prime}\right)\right|_{\sigma-\text { rep. }} \mathrm{d} \sigma
$$

where $\bar{B}_{K}$ is defined through $B_{K}$ as follows (recall that $B_{K}$ is a function of $\left.\left(\left|\mathbf{v}-\mathbf{v}^{\prime}\right|,\left|\mathbf{v}-\mathbf{v}_{*}^{\prime}\right|\right)\right)$ :

$$
\begin{aligned}
& B_{K}\left(\mathbf{v}-\mathbf{v}_{*}, \omega\right)=\left.\widetilde{B}_{K}\left(\left|\mathbf{v}-\mathbf{v}^{\prime}\right|,\left|\mathbf{v}-\mathbf{v}_{*}^{\prime}\right|\right)\right|_{\omega-\text { rep. }} \\
& \bar{B}_{K}\left(\mathbf{v}-\mathbf{v}_{*}, \sigma\right)=\left.\frac{\left|\mathbf{v}-\mathbf{v}_{*}\right|}{2\left|\mathbf{v}-\mathbf{v}^{\prime}\right|} \widetilde{B}_{K}\left(\left|\mathbf{v}-\mathbf{v}^{\prime}\right|,\left|\mathbf{v}-\mathbf{v}_{*}^{\prime}\right|\right)\right|_{\sigma-\text { rep. }}
\end{aligned}
$$

The $\sigma$-representation (4.1) in many cases is convenient than the $\omega$-representation (1.2), but since it is non-linear and non-smooth in $\left(\mathbf{v}, \mathbf{v}_{*}\right)$, one needs to go back to $\omega$-representation $(1.2)$ when proving some integral identities. For instance this can be seen in the proof of the following formula of change of variables for $\sigma$-representation:

$$
\begin{aligned}
& \int_{\mathbb{R}^{3} \times \mathbb{S}^{2}} \Psi\left(\left|\mathbf{v}-\mathbf{v}_{*}\right|, \mathbf{n} \cdot \sigma, \mathbf{v}^{\prime}, \mathbf{v}_{*}^{\prime}\right) \mathrm{d} \sigma \mathrm{d} \mathbf{v}_{*} \\
& =\int_{0}^{\pi} \frac{\sin (\theta)}{\cos ^{3}(\theta / 2)} \mathrm{d} \theta \int_{\mathbb{R}^{3}} \mathrm{~d} \mathbf{v}_{*} \int_{\mathbb{S}^{1}(\mathbf{n})} \Psi\left(\frac{\left|\mathbf{v}-\mathbf{v}_{*}\right|}{\cos (\theta / 2)}, \cos (\theta), \mathbf{v}-\left|\mathbf{v}-\mathbf{v}_{*}\right| \frac{\sin (\theta / 2)}{\cos (\theta / 2)} \tilde{\sigma}, \mathbf{v}_{*}\right) \mathrm{d} \tilde{\sigma}
\end{aligned}
$$

where $\mathbf{n}=\frac{\mathbf{v}-\mathbf{v}_{*}}{\left|\mathbf{v}-\mathbf{v}_{*}\right|}, \Psi$ is any nonnegative Lebesgue measurable functions on $\mathbb{R}_{\geq 0} \times[-1,1] \times$ $\mathbb{R}^{3} \times \mathbb{R}^{3}, \mathbb{S}^{1}(\mathbf{n})=\left\{\tilde{\sigma} \in \mathbb{S}^{2} \mid \tilde{\sigma} \perp \mathbf{n}\right\}$ and $\mathrm{d} \tilde{\sigma}$ is the circle measure element on $\mathbb{S}^{1}(\mathbf{n})$.

The proof of (4.6) is just several elementary changes of variables:

(1) take reflection $\sigma \rightarrow-\sigma$, then use $\left|\mathbf{v}-\mathbf{v}_{*}\right|=\left|\mathbf{v}^{\prime}-\mathbf{v}_{*}^{\prime}\right|, \mathbf{v}_{*}=\mathbf{v}^{\prime}+\mathbf{v}_{*}^{\prime}-\mathbf{v}$ to write the integrand as a function of $\left(\mathbf{v}^{\prime}, \mathbf{v}_{*}^{\prime}\right)$ (with $\mathbf{v}$ fixed): $\Psi=\Psi\left(\left|\mathbf{v}^{\prime}-\mathbf{v}_{*}^{\prime}\right|, \frac{\left(\mathbf{v}^{\prime}-\mathbf{v}_{*}^{\prime}\right) \cdot\left(\mathbf{v}^{\prime}+\mathbf{v}_{*}^{\prime}-2 \mathbf{v}\right)}{\left|\mathbf{v}^{\prime}-\mathbf{v}_{*}^{\prime}\right|^{2}}, \mathbf{v}_{*}^{\prime}, \mathbf{v}^{\prime}\right)$;

(2) use the formula (4.2), then change variables $\mathbf{v}_{*}=\mathbf{v}-\mathbf{z}, \mathbf{z}=r \sigma$;

(3) change variables $r=\frac{\rho}{\sigma \cdot \omega}$ for $\sigma \cdot \omega>0, \rho \omega=\mathbf{z}=\mathbf{v}-\mathbf{v}_{*}$, then denote again $\mathbf{n}=\frac{\mathbf{v}-\mathbf{v}_{*}}{\left|\mathbf{v}-\mathbf{v}_{*}\right|}$;

(4) change variables $\sigma=\mathbf{n} \cos (\theta)+\sin (\theta) \widetilde{\sigma}, \tilde{\sigma} \in \mathbb{S}^{1}(\mathbf{n})$, etc. 
Accordingly we compute

$$
\begin{aligned}
& \int_{\mathbb{R}^{3} \times \mathbb{S}^{2}} \Psi\left(\left|\mathbf{v}-\mathbf{v}_{*}\right|, \mathbf{n} \cdot \sigma, \mathbf{v}^{\prime}, \mathbf{v}_{*}^{\prime}\right) \mathrm{d} \sigma \mathrm{d} \mathbf{v}_{*}=\int_{\mathbb{R}^{3} \times \mathbb{S}^{2}} 2|\mathbf{n} \cdot \omega| \Psi\left(\left|\mathbf{v}-\mathbf{v}_{*}\right|, 2(\mathbf{n} \cdot \omega)^{2}-1, \mathbf{v}_{*}^{\prime}, \mathbf{v}^{\prime}\right) \mathrm{d} \omega \mathrm{d} \mathbf{v}_{*} \\
& =4 \int_{\mathbb{S}^{2} \times \mathbb{S}^{2}} \mathbf{1}_{\{\sigma \cdot \omega>0\}}|\sigma \cdot \omega| \mathrm{d} \omega \mathrm{d} \sigma \int_{0}^{\infty} r^{2} \Psi\left(r, 2(\sigma \cdot \omega)^{2}-1, \mathbf{v}-r \sigma+r(\sigma \cdot \omega) \omega, \mathbf{v}-r(\sigma \cdot \omega) \omega\right) \mathrm{d} r \\
& =4 \int_{\mathbb{R}^{3}} \mathrm{~d} \mathbf{v}_{*} \int_{\mathbb{S}^{2}} \mathbf{1}_{\{\sigma \cdot \mathbf{n}>0\}} \frac{1}{(\sigma \cdot \mathbf{n})^{2}} \Psi\left(\frac{\left|\mathbf{v}-\mathbf{v}_{*}\right|}{\sigma \cdot \mathbf{n}}, 2(\sigma \cdot \mathbf{n})^{2}-1, \mathbf{v}-\frac{\left|\mathbf{v}-\mathbf{v}_{*}\right|}{\sigma \cdot \mathbf{n}} \sigma+\mathbf{v}-\mathbf{v}_{*}, \mathbf{v}_{*}\right) \mathrm{d} \sigma \\
& =4 \int_{\mathbb{R}^{3}} \mathrm{~d} \mathbf{v}_{*} \int_{0}^{\pi / 2} \frac{\sin (\theta)}{\cos ^{2}(\theta)} \mathrm{d} \theta \int_{\mathbb{S}^{1}(\mathbf{n})} \Psi\left(\frac{\left|\mathbf{v}-\mathbf{v}_{*}\right|}{\cos (\theta)}, 2 \cos ^{2}(\theta)-1, \mathbf{v}-\left|\mathbf{v}-\mathbf{v}_{*}\right| \frac{\sin (\theta)}{\cos (\theta)} \widetilde{\sigma}, \mathbf{v}_{*}\right) \mathrm{d} \widetilde{\sigma}
\end{aligned}
$$

which is equal to the right hand side of (4.6).

As an application of the formula (4.6) we prove the following lemma which is also used in the proof of Proposition 4.2 .

Lemma 4.1. Let $\alpha>0$ be a constant and $0 \leq f \in L^{1}\left(\mathbb{R}^{3}\right)$. Then

$$
\int_{\mathbb{R}^{3} \times \mathbb{S}^{2}}\left|\mathbf{v}-\mathbf{v}_{*}\right|\left|\mathbf{v}-\mathbf{v}^{\prime}\right| f\left(\mathbf{v}^{\prime}\right) e^{-\alpha\left|\mathbf{v}_{*}^{\prime}\right|} \mathrm{d} \sigma \mathrm{d} \mathbf{v}_{*} \leq 8 \pi \frac{1+2 \alpha}{\alpha^{2}}\langle\mathbf{v}\rangle\|f\|_{L^{1}}
$$

Proof. Using the formula (4.6) and $|\mathbf{v}-\mathbf{u}| \geq|| \mathbf{v}|-| \mathbf{u}||$ we have

$$
\begin{aligned}
& \int_{\mathbb{R}^{3} \times \mathbb{S}^{2}}\left|\mathbf{v}-\mathbf{v}_{*}\right|\left|\mathbf{v}-\mathbf{v}^{\prime}\right| f\left(\mathbf{v}^{\prime}\right) e^{-\alpha\left|\mathbf{v}_{*}^{\prime}\right|} \mathrm{d} \sigma \mathrm{d} \mathbf{v}_{*} \\
& =\int_{0}^{\pi} \frac{\sin (\theta)}{\cos ^{3}(\theta / 2)} \mathrm{d} \theta \int_{\mathbb{R}^{3}} \mathrm{~d} \mathbf{v}_{*} \int_{\mathbb{S}^{1}(\mathbf{n})} \frac{\left|\mathbf{v}-\mathbf{v}_{*}\right|^{2}}{\cos (\theta / 2)} f\left(\mathbf{v}_{*}\right) \exp \left(-\alpha|\mathbf{v}-| \mathbf{v}-\mathbf{v}_{*}\left|\frac{\sin (\theta / 2)}{\cos (\theta / 2)} \tilde{\sigma}\right|\right) \mathrm{d} \tilde{\sigma} \\
& \leq 8 \pi \int_{\mathbb{R}^{3}}\left|\mathbf{v}-\mathbf{v}_{*}\right|^{2} f\left(\mathbf{v}_{*}\right) \mathrm{d} \mathbf{v}_{*} \int_{0}^{\pi / 2} \frac{\sin (\theta)}{\cos ^{3}(\theta)} \exp \left(-\alpha|| \mathbf{v}|-| \mathbf{v}-\mathbf{v}_{*}\left|\frac{\sin (\theta)}{\cos (\theta)}\right|\right) \mathrm{d} \theta,
\end{aligned}
$$

and for the inner integral we compute (changing variable $\theta=\arctan (t)$, etc.)

$$
\int_{0}^{\pi / 2}\{\cdots\} \mathrm{d} \theta=\frac{1}{\left|\mathbf{v}-\mathbf{v}_{*}\right|^{2}} \int_{-|\mathbf{v}|}^{\infty}(t+|\mathbf{v}|) e^{-\alpha|t|} \mathrm{d} t \leq \frac{1}{\left|\mathbf{v}-\mathbf{v}_{*}\right|^{2}}\left(\frac{1}{\alpha^{2}}+2|\mathbf{v}| \frac{1}{\alpha}\right) .
$$

Inserting this into the above inequality gives (4.7).

The following proposition is established for approximate solutions of Eq.(1.1) for arbitrary initial data, in particular it includes anisotropic initial data.

Proposition 4.2. Let $B\left(\mathbf{v}-\mathbf{v}_{*}, \omega\right)$ satisfy Assumption 1.1 and let $p_{1}, p_{2}, \delta$ be constants satisfying

$$
2<p_{1}<\infty, \quad \frac{4+2 \eta}{3}<p_{2}<\infty, \quad \delta>0
$$

Let $\left\{B_{K}\left(\mathbf{v}-\mathbf{v}_{*}, \omega\right)\right\}_{K \in \mathbb{N}}$ be a sequence of approximation of $B\left(\mathbf{v}-\mathbf{v}_{*}, \omega\right)$ satisfying

$$
\min \left\{B\left(\mathbf{v}-\mathbf{v}_{*}, \omega\right), K\left|\mathbf{v}-\mathbf{v}^{\prime}\right|^{2}\left|\mathbf{v}-\mathbf{v}_{*}^{\prime}\right|\right\} \leq B_{K}\left(\mathbf{v}-\mathbf{v}_{*}, \omega\right) \leq B\left(\mathbf{v}-\mathbf{v}_{*}, \omega\right)
$$


for all $K \in \mathbb{N},\left(\mathbf{v}, \mathbf{v}_{*}, \omega\right) \times \mathbb{R}^{3} \times \mathbb{R}^{3} \times \mathbb{S}^{2}$. Given any $N>0, E>0$, let $\Omega(\mathbf{v})=f_{\mathrm{be}}\left(|\mathbf{v}|^{2} / 2\right)$ be given in Proposition 2.2 with $f_{\mathrm{be}}(x)$ the regular part of the equilibrium $F_{\mathrm{be}}$ that has the mass and energy $N, E$. Let $\left\{f_{0}^{K}\right\}_{K \in \mathbb{N}} \subset L_{2}^{1}\left(\mathbb{R}^{3}\right)$ be a nonnegative sequence satisfying

$$
\int_{\mathbb{R}^{3}}\left(1, \mathbf{v},|\mathbf{v}|^{2} / 2\right) f_{0}^{K}(\mathbf{v}) \mathrm{d} \mathbf{v}=4 \sqrt{2}(N, 0, E) \quad \forall K \in \mathbb{N}
$$

let $\left\{f^{K}\right\}_{K \in \mathbb{N}} \subset L^{\infty}\left([0, \infty) ; L_{2}^{1}\left(\mathbb{R}^{3}\right)\right) \cap L^{\infty}\left([1, \infty) ; L_{4}^{1}\left(\mathbb{R}^{3}\right)\right)$ be a sequence of approximate solutions (in terms of Definition 3.1) of Eq.(1.1) on $\mathbb{R}^{3} \times[0, \infty)$ corresponding to the approximate kernels $\left\{B_{K}\right\}_{K \in \mathbb{N}}$ satisfying $\left.f^{K}\right|_{t=0}=f_{0}^{K}$ for all $K \in \mathbb{N}$, and suppose for some constants $S_{0}>0,0<$ $C_{0}<\infty$,

$$
\inf _{K \in \mathbb{N}, t \geq 1} S\left(f^{K}(t)\right) \geq S_{0}, \quad \sup _{K \in \mathbb{N}, t \geq 1}\left\|f^{K}(t)\right\|_{L_{2+q_{2}}^{1}} \leq C_{0}
$$

where $q_{2}=p_{2} /\left(p_{2}-1\right)$. Let $\mathcal{E}(\mathbf{v})=\alpha_{0} e^{-\beta_{0}|\mathbf{v}|}$ where $\alpha_{0}=\alpha_{0}(N, E)>0, \beta_{0}=\beta_{0}(N, E)>0$ are given through the moment equations

$$
\int_{\mathbb{R}^{3}}\left(1,|\mathbf{v}|^{2} / 2\right) \mathcal{E}(\mathbf{v}) \mathrm{d} \mathbf{v}=4 \pi \sqrt{2}(N, E) .
$$

Let

$$
F^{K}(\mathbf{v}, t)=\left(1-e^{-t^{\delta}}\right) f^{K}(\mathbf{v}, t)+e^{-t^{\delta}} \mathcal{E}(\mathbf{v}), \quad(\mathbf{v}, t) \in \mathbb{R}^{3} \times[0, \infty) .
$$

Then there are constants $0<C_{i}<\infty(i=1,2,3,4)$ that depend only on $N, E, S_{0}, C_{0}, b_{0}, \eta, p_{1}, p_{2}$, and $\delta$ such that

(I) For any $K \in \mathbb{N}, 0<T<\infty$, the entropy $t \mapsto S\left(F^{K}(t)\right)$ is absolutely continuous on $[0, T]$ and

$$
\frac{\mathrm{d}}{\mathrm{d} t}\left(S(\Omega)-S\left(F^{K}(t)\right)\right) \leq-D_{K}\left(F^{K}(t)\right)+C_{1} t^{\delta} e^{-t^{\delta}}, \quad \text { a.e. } t \in[1, \infty) .
$$

(II) Let $G^{K}=\frac{F^{K}}{1+F^{K}}$. Then for any $K \in \mathbb{N}$

$$
\mathcal{D}_{2}\left(G^{K}(t)\right) \leq C_{2}\left(\left(t^{\delta\left(p_{1}-1\right)} K^{-1} D_{K}\left(F^{K}(t)\right)^{\frac{1}{p_{1}}}+\left(t^{\delta} D_{K}\left(F^{K}(t)\right)\right)^{\frac{1}{p_{2}}}\right) \quad \forall t \in[1, \infty) .\right.
$$

(III) There is a constant $C_{3}>0$ such that for the function $\Psi(y)=C_{3}\left(y^{\frac{1}{10\left(p_{1} \vee p_{2}\right)}}+y^{\frac{1}{4}}\right), y \in$ $[0, \infty)$, it holds

$$
\frac{\mathrm{d}}{\mathrm{d} t}\left(S(\Omega)-S\left(F^{K}(t)\right)\right) \leq-t^{-\delta} \Psi^{-1}\left(S(\Omega)-S\left(F^{K}(t)\right)\right)+C_{4} t^{\delta} e^{-t^{\delta}} \quad \text { a.e. } t \in[1, T]
$$

for all $1<T<\infty$ and all $K \geq T^{\delta\left(p_{1}-2\right)}$, where $\Psi^{-1}(u)$ is the inverse function of $\Psi(y)$.

Proof. First we see from the above assumptions that $F^{K}$ satisfy also (4.10), i.e.

$$
\int_{\mathbb{R}^{3}}\left(1, \mathbf{v},|\mathbf{v}|^{2} / 2\right) F^{K}(\mathbf{v}, t) \mathrm{d} \mathbf{v}=4 \sqrt{2}(N, 0, E) \quad \forall t \geq 0, \forall K \in \mathbb{N}
$$


and so by Proposition 2.2 we have $S(\Omega)-S\left(F^{K}(t)\right) \geq 0$ for all $t \geq 0, K \in \mathbb{N}$. Next by (4.12), concaveness (1.28), increase of $t \mapsto S\left(f^{K}(t)\right)$, and (4.11) we have

$$
S\left(F^{k}(t)\right) \geq\left(1-e^{-t^{\delta}}\right) S\left(f^{K}(t)\right)+e^{-t^{\delta}} S(\mathcal{E}) \geq\left(1-e^{-t^{\delta}}\right) S_{0}+e^{-t^{\delta}} S(\mathcal{E}) \quad \forall t \geq 1
$$

which implies that

$$
\inf _{K \in \mathbb{N}, t \geq 1} S\left(F^{k}(t)\right) \geq \min \left\{S_{0}, S(\mathcal{E})\right\}>0
$$

Also we have

$$
\sup _{K \in \mathbb{N}, t \geq 1}\left\|F^{K}(t)\right\|_{L_{4}^{1}} \leq \max \left\{C_{0},\|\mathcal{E}\|_{L_{4}^{1}}\right\}<\infty .
$$

We note also that, since $f^{K}$ satisfy the integrability assumption (3.1), there are no problems of integrability in the following derivation. To simplify notations we denote for any given $K \in \mathbb{N}$

$$
f(\mathbf{v}, t)=f^{K}(\mathbf{v}, t), \quad F(\mathbf{v}, t)=F^{K}(\mathbf{v}, t), \quad G(\mathbf{v}, t)=\frac{F(\mathbf{v}, t)}{1+F(\mathbf{v}, t)} .
$$

In the following all constants $0<C_{i}<\infty(i=1,2, \ldots, 27)$ depend only on $N, E, S_{0}, C_{0}, b_{0}, \eta, p_{1}, p_{2}$, and $\delta$.

(I): Since

$$
0 \leq \log \left(\frac{1}{G(\mathbf{v}, t)}\right) \leq \log \left(1+\frac{1}{\alpha_{0}} e^{t^{\delta}+\beta_{0}|\mathbf{v}|}\right) \leq C_{5}\left(1+t^{\delta}\right)\langle\mathbf{v}\rangle
$$

it follows that for almost every $\mathbf{v} \in \mathbb{R}^{3}, t \mapsto s(F(\mathbf{v}, t)$ ) is absolutely continuous on [0,T] (for all $0<T<\infty)$ and so for all $0 \leq t_{1}<t_{2}<\infty$

$$
\begin{aligned}
& s\left(F\left(\mathbf{v}, t_{2}\right)\right)-s\left(F\left(\mathbf{v}, t_{1}\right)\right) \\
& =\int_{t_{1}}^{t_{2}} \log \left(\frac{1}{G(\mathbf{v}, \tau)}\right)\left(\delta \tau^{\delta-1} e^{-\tau^{\delta}}(f(\mathbf{v}, \tau)-\mathcal{E}(\mathbf{v}))+\left(1-e^{-\tau^{\delta}}\right) Q_{K}(f)(\mathbf{v}, \tau)\right) \mathrm{d} \tau, \\
& S\left(F\left(t_{2}\right)\right)-S\left(F\left(t_{1}\right)\right) \\
& =\int_{t_{1}}^{t_{2}} \mathrm{~d} \tau \int_{\mathbb{R}^{3}} \log \left(\frac{1}{G(\mathbf{v}, \tau)}\right)\left(\delta \tau^{\delta-1} e^{-\tau^{\delta}}(f(\mathbf{v}, \tau)-\mathcal{E}(\mathbf{v}))+\left(1-e^{-\tau^{\delta}}\right) Q_{K}(f)(\mathbf{v}, \tau)\right) \mathrm{d} \mathbf{v} .
\end{aligned}
$$

This implies that for any $0<T<\infty$ the entropy $t \mapsto S\left(F^{K}(t)\right)$ is absolutely continuous on $[0, T]$ and for almost every $t \in[0, \infty)$

$$
\frac{\mathrm{d}}{\mathrm{d} t}(S(\Omega)-S(F(t)))=\delta t^{\delta-1} e^{-t^{\delta}} \int_{\mathbb{R}^{3}}(\mathcal{E}-f) \log \left(\frac{1}{G}\right) \mathrm{d} \mathbf{v}-\left(1-e^{-t^{\delta}}\right) \int_{\mathbb{R}^{3}} Q_{K}(f) \log \left(\frac{1}{G}\right) \mathrm{d} \mathbf{v} .
$$

With the $\sigma$-representation we have

$$
\begin{aligned}
& \int_{\mathbb{R}^{3}} Q_{K}(f)(\mathbf{v}, t) \log \left(\frac{1}{G(\mathbf{v}, t)}\right) \mathrm{d} \mathbf{v} \\
& =\frac{1}{4} \int_{\mathbb{R}^{3} \times \mathbb{R}^{3} \times \mathbb{S}^{2}} \bar{B}_{K}\left(\mathbf{v}-\mathbf{v}_{*}, \sigma\right)\left(f^{\prime} f_{*}^{\prime}\left(1+f+f_{*}\right)-f f_{*}\left(1+f^{\prime}+f_{*}^{\prime}\right)\right) \log \left(\frac{G^{\prime} G_{*}^{\prime}}{G G_{*}}\right) \mathrm{d} \mathbf{v} \mathrm{d} \mathbf{v}_{*} \mathrm{~d} \sigma .
\end{aligned}
$$


Now we will prove that with an error $O\left(t^{\delta} e^{-t^{\delta}}\right)$ the right hand side of (4.20) is approximately less than $-D_{K}(F(t))$. Recalling $(3.5),(4.3),(4.4),(4.5)$ we write (with the $\sigma$-representation)

$$
D_{K}(F)=\frac{1}{4} \int_{\mathbb{R}^{3} \times \mathbb{R}^{3} \times \mathbb{S}^{2}} \bar{B}_{K} \cdot\left(F^{\prime} F_{*}^{\prime}\left(1+F+F_{*}\right)-F F_{*}\left(1+F^{\prime}+F_{*}^{\prime}\right)\right) \log \left(\frac{G^{\prime} G_{*}^{\prime}}{G G_{*}}\right) \mathrm{d} \mathbf{v} \mathrm{d} \mathbf{v}_{*} \mathrm{~d} \sigma .
$$

Before going on we note that from the assumption (4.9) and relation between the $\omega$-representation and $\sigma$-representation we have for the $\sigma$-representation that

$$
\frac{\left|\mathbf{v}-\mathbf{v}_{*}\right|}{2(4 \pi)^{2}} \min \left\{\Phi\left(\left|\mathbf{v}-\mathbf{v}^{\prime}\right|,\left|\mathbf{v}-\mathbf{v}_{*}^{\prime}\right|\right),(4 \pi)^{2} K\left|\mathbf{v}-\mathbf{v}^{\prime}\right|\left|\mathbf{v}-\mathbf{v}_{*}^{\prime}\right|\right\} \leq \bar{B}_{K}\left(\mathbf{v}-\mathbf{v}_{*}, \sigma\right) \leq \frac{\left|\mathbf{v}-\mathbf{v}_{*}\right|}{2(4 \pi)^{2}}
$$

For the convex combination $F=\left(1-e^{-t^{\delta}}\right) f+e^{-t^{\delta}} \mathcal{E}$ we compute

$$
\begin{aligned}
& F^{\prime} F_{*}^{\prime}\left(1+F+F_{*}\right)-F F_{*}\left(1+F^{\prime}+F_{*}^{\prime}\right) \\
& =\left(1-e^{-t^{\delta}}\right)^{3}\left(f^{\prime} f_{*}^{\prime}\left(1+f+f_{*}\right)-f f_{*}\left(1+f^{\prime}+f_{*}^{\prime}\right)\right) \\
& +\left(1-e^{-t^{\delta}}\right)^{2} e^{-t^{\delta}}\left(f^{\prime} f_{*}^{\prime}\left(1+\mathcal{E}+\mathcal{E}_{*}\right)-f f_{*}\left(1+\mathcal{E}^{\prime}+\mathcal{E}_{*}^{\prime}\right)\right) \\
& +\left(1-e^{-t^{\delta}}\right)^{2} e^{-t^{\delta}}\left(\left(f^{\prime} \mathcal{E}_{*}^{\prime}+f_{*}^{\prime} \mathcal{E}^{\prime}\right)-\left(f \mathcal{E}_{*}+f_{*} \mathcal{E}\right)\right) \\
& +\left(1-e^{-t^{\delta}}\right)^{2} e^{-t^{\delta}}\left(\left(f^{\prime} \mathcal{E}_{*}^{\prime}+f_{*}^{\prime} \mathcal{E}^{\prime}\right)\left(f+f_{*}\right)-\left(f \mathcal{E}_{*}+f_{*} \mathcal{E}\right)\left(f^{\prime}+f_{*}^{\prime}\right)\right) \\
& +\left(1-e^{-t^{\delta}}\right) e^{-2 t^{\delta}}\left(\left(f^{\prime} \mathcal{E}_{*}^{\prime}+f_{*}^{\prime} \mathcal{E}^{\prime}\right)\left(1+\mathcal{E}+\mathcal{E}_{*}\right)-\left(f \mathcal{E}_{*}+f_{*} \mathcal{E}\right)\left(1+\mathcal{E}^{\prime}+\mathcal{E}_{*}^{\prime}\right)\right) \\
& +\left(1-e^{-t^{\delta}}\right) e^{-2 t^{\delta}}\left(\mathcal{E}^{\prime} \mathcal{E}_{*}^{\prime}-\mathcal{E}_{*}\right) \\
& +\left(1-e^{-t^{\delta}}\right) e^{-2 t^{\delta}}\left(\mathcal{E}^{\prime} \mathcal{E}_{*}^{\prime}\left(f+f_{*}\right)-\mathcal{E} \mathcal{E}_{*}\left(f^{\prime}+f_{*}^{\prime}\right)\right) \\
& +e^{-3 t^{\delta}}\left(\mathcal{E}^{\prime} \mathcal{E}_{*}^{\prime}\left(1+\mathcal{E}+\mathcal{E}_{*}\right)-\mathcal{E}_{*}\left(1+\mathcal{E}^{\prime}+\mathcal{E}_{*}^{\prime}\right)\right) \\
& :=\left(1-e^{-t^{\delta}}\right)^{3}\left(f^{\prime} f_{*}^{\prime}\left(1+f+f_{*}\right)-f f_{*}\left(1+f^{\prime}+f_{*}^{\prime}\right)\right)+\Psi_{1}+\Psi_{2}+\cdots+\Psi_{7} \\
& \Longrightarrow D_{K}(F)=\left(1-e^{-t^{\delta}}\right)^{3} \int_{\mathbb{R}^{3}} Q_{K}(f)(\mathbf{v}, t) \log \left(\frac{1}{G(\mathbf{v}, t)}\right) \mathrm{d} \mathbf{v} \\
& +\frac{1}{4} \sum_{j=1}^{7} \int_{\mathbb{R}^{3} \times \mathbb{R}^{3} \times \mathbb{S}^{2}} \bar{B}_{K} \cdot \Psi_{j} \log \left(\frac{G^{\prime} G_{*}^{\prime}}{G G_{*}}\right) \mathrm{d} \mathbf{v} \mathrm{d} \mathbf{v}_{*} \mathrm{~d} \sigma .
\end{aligned}
$$

Comparing this with (4.20) we obtain

$$
\begin{aligned}
& \frac{\mathrm{d}}{\mathrm{d} t}(S(\Omega)-S(F(t)))=\delta t^{\delta-1} e^{-t^{\delta}} \int_{\mathbb{R}^{3}}(\mathcal{E}-f) \log \left(\frac{1}{G}\right) \mathrm{d} \mathbf{v}-\frac{1}{\left(1-e^{-t^{\delta}}\right)^{2}} D_{K}(F) \\
& +\frac{1}{4\left(1-e^{-t^{\delta}}\right)^{2}} \sum_{j=1}^{7} \int_{\mathbb{R}^{3} \times \mathbb{R}^{3} \times \mathbb{S}^{2}} \bar{B}_{K} \cdot \Psi_{j} \log \left(\frac{G^{\prime} G_{*}^{\prime}}{G G_{*}}\right) \mathrm{d} \mathbf{v} \mathrm{d} \mathbf{v}_{*} \mathrm{~d} \sigma, \quad \text { a.e. } t \geq 1 .
\end{aligned}
$$

By definition of $G$ we have

$$
t^{\delta-1} e^{-t^{\delta}} \int_{\mathbb{R}^{3}}(\mathcal{E}-f) \log \left(\frac{1}{G}\right) \mathrm{d} \mathbf{v} \leq C_{6} t^{2 \delta-1} e^{-t^{\delta}} \int_{\mathbb{R}^{3}} \mathcal{E}(\mathbf{v})(1+|\mathbf{v}|) \mathrm{d} \mathbf{v} \leq C_{7} t^{\delta} e^{-t^{\delta}}, \quad t \geq 1 .
$$


From (4.19) we have

$$
\left|\log \left(\frac{G\left(\mathbf{v}^{\prime}, t\right) G\left(\mathbf{v}_{*}^{\prime}, t\right)}{G(\mathbf{v}, t) G\left(\mathbf{v}_{*}, t\right)}\right)\right| \leq C_{8} t^{\delta} \sqrt{1+|\mathbf{v}|^{2}+\left|\mathbf{v}_{*}\right|^{2}}, \quad t \geq 1 .
$$

Also from $\left|\mathbf{v}-\mathbf{v}_{*}\right|=\left|\mathbf{v}^{\prime}-\mathbf{v}_{*}^{\prime}\right|,|\mathbf{v}|^{2}+\left|\mathbf{v}_{*}\right|^{2}=\left|\mathbf{v}^{\prime}\right|^{2}+\left|\mathbf{v}_{*}^{\prime}\right|^{2}$ we have

$$
\left|\mathbf{v}-\mathbf{v}_{*}\right| \sqrt{1+|\mathbf{v}|^{2}+\left|\mathbf{v}_{*}\right|^{2}} \leq \min \left\{\langle\mathbf{v}\rangle^{2}\left\langle\mathbf{v}_{*}\right\rangle^{2},\left\langle\mathbf{v}^{\prime}\right\rangle^{2}\left\langle\mathbf{v}_{*}^{\prime}\right\rangle^{2}\right\}
$$

From (4.21), (4.24),(4.25) we obtain

$$
\begin{aligned}
& \frac{1}{4\left(1-e^{-t^{\delta}}\right)^{2}} \sum_{1 \leq j \leq 7, j \neq 3} \int_{\mathbb{R}^{3} \times \mathbb{R}^{3} \times \mathbb{S}^{2}} \bar{B}_{K}\left|\Psi_{j}\right|\left|\log \left(\frac{G^{\prime} G_{*}^{\prime}}{G G_{*}}\right)\right| \mathrm{d} \mathbf{v} \mathrm{d} \mathbf{v}_{*} \mathrm{~d} \sigma \\
& \leq C_{9} e^{-t^{\delta}} t^{\delta}\left(\left(\|f(t)\|_{L_{2}^{1}}+\|\mathcal{E}\|_{L_{2}^{1}}\right)^{2}+\int_{\mathbb{R}^{3} \times \mathbb{R}^{3} \times \mathbb{S}^{2}} \widetilde{\mathcal{E}}^{\prime} \widetilde{\mathcal{E}}_{*}^{\prime} f \mathrm{~d} \mathbf{v} \mathrm{d} \mathbf{v}_{*} \mathrm{~d} \sigma\right), \quad t \geq 1
\end{aligned}
$$

where $\widetilde{\mathcal{E}}(\mathbf{v})=\langle\mathbf{v}\rangle^{2} \mathcal{E}(\mathbf{v})$. By definition of $\mathcal{E}$ we have $\widetilde{\mathcal{E}}(\mathbf{v}) \leq \alpha_{0}\left(1+4 / \beta_{0}\right)^{2} e^{-\frac{\beta_{0}}{2}|\mathbf{v}|}$. Since $\left|\mathbf{v}^{\prime}\right|+\left|\mathbf{v}_{*}^{\prime}\right| \geq \sqrt{\left|\mathbf{v}^{\prime}\right|^{2}+\left|\mathbf{v}_{*}^{\prime}\right|^{2}}=\sqrt{|\mathbf{v}|^{2}+\left|\mathbf{v}_{*}\right|^{2}} \geq\left|\mathbf{v}_{*}\right|$, this gives $\widetilde{\mathcal{E}}^{\prime} \widetilde{\mathcal{E}}_{*}^{\prime} \leq C_{10} e^{-\frac{\beta_{0}}{2}\left|\mathbf{v}_{*}\right|}$. So

$$
\int_{\mathbb{R}^{3} \times \mathbb{R}^{3} \times \mathbb{S}^{2}} \widetilde{\mathcal{E}}^{\prime} \widetilde{\mathcal{E}}_{*}^{\prime} f \mathrm{~d} \mathbf{v} \mathrm{d} \mathbf{v}_{*} \mathrm{~d} \sigma \leq C_{10} 4 \pi \int_{\mathbb{R}^{3} \times \mathbb{R}^{3}} e^{-\frac{\beta_{0}}{2}\left|\mathbf{v}_{*}\right|} f(\mathbf{v}, t) \mathrm{d} \mathbf{v} \mathrm{d} \mathbf{v}_{*}=C_{11}\|f(t)\|_{L^{1}} .
$$

Now we estimate the integral involving $\Psi_{3}$. We write

$$
\begin{aligned}
& \left(f^{\prime} \mathcal{E}_{*}^{\prime}+f_{*}^{\prime} \mathcal{E}^{\prime}\right)\left(f+f_{*}\right)-\left(f \mathcal{E}_{*}+f_{*} \mathcal{E}\right)\left(f^{\prime}+f_{*}^{\prime}\right) \\
& =f f^{\prime}\left(\mathcal{E}_{*}^{\prime}-\mathcal{E}_{*}\right)+f_{*} f^{\prime}\left(\mathcal{E}_{*}^{\prime}-\mathcal{E}\right)+f f_{*}^{\prime}\left(\mathcal{E}^{\prime}-\mathcal{E}_{*}\right)+f_{*} f_{*}^{\prime}\left(\mathcal{E}^{\prime}-\mathcal{E}\right) .
\end{aligned}
$$

By change of variables $\left(\mathbf{v}, \mathbf{v}_{*}\right) \rightarrow\left(\mathbf{v}_{*}, \mathbf{v}\right)$ and $\sigma \rightarrow-\sigma$ and notice that $\bar{B}_{K}\left(\mathbf{v}-\mathbf{v}_{*}, \sigma\right)=\bar{B}_{K}\left(\mathbf{v}_{*}-\right.$ $\mathbf{v}, \sigma), \bar{B}_{K}\left(\mathbf{v}-\mathbf{v}_{*},-\sigma\right)=\bar{B}_{K}\left(\mathbf{v}-\mathbf{v}_{*}, \sigma\right)($ see $(4.5))$, we have

$$
\begin{aligned}
& \frac{1}{4\left(1-e^{-t^{\delta}}\right)^{2}} \int_{\mathbb{R}^{3} \times \mathbb{R}^{3} \times \mathbb{S}^{2}} \bar{B}_{K} \cdot \Psi_{3} \log \left(\frac{G^{\prime} G_{*}^{\prime}}{G G_{*}}\right) \mathrm{d} \mathbf{v} \mathrm{d} \mathbf{v}_{*} \mathrm{~d} \sigma \\
& =e^{-t^{\delta}} \int_{\mathbb{R}^{3} \times \mathbb{R}^{3} \times \mathbb{S}^{2}} \bar{B}_{K} \cdot f f^{\prime}\left(\mathcal{E}_{*}^{\prime}-\mathcal{E}_{*}\right) \log \left(\frac{G^{\prime} G_{*}^{\prime}}{G G_{*}}\right) \mathrm{d} \mathbf{v} \mathrm{d} \mathbf{v}_{*} \mathrm{~d} \sigma \\
& \leq\left. 2 e^{-t^{\delta}} \int_{\mathbb{R}^{3} \times \mathbb{R}^{3} \times \mathbb{S}^{2}} \bar{B}_{K} \cdot f f^{\prime}\left(\mathcal{E}_{*}^{\prime}-\mathcal{E}_{*}\right)_{+} \log \left(\frac{G^{\prime} G_{*}^{\prime}}{G G_{*}}\right)\right|_{G^{\prime} G_{*}^{\prime}>G G_{*}} \mathrm{~d} \mathbf{v} \mathrm{d} \mathbf{v}_{*} \mathrm{~d} \sigma \\
& \leq \frac{C_{12}}{(4 \pi)^{2}} t^{\delta} e^{-t^{\delta}} \int_{\mathbb{R}^{3} \times \mathbb{R}^{3} \times \mathbb{S}^{2}}\left|\mathbf{v}-\mathbf{v}_{*}\right| f f^{\prime}\left(\mathcal{E}_{*}^{\prime}-\mathcal{E}_{*}\right)_{+} \sqrt{1+|\mathbf{v}|^{2}+\left|\mathbf{v}_{*}\right|^{2}} \mathrm{~d} \mathbf{v} \mathrm{d} \mathbf{v}_{*} \mathrm{~d} \sigma .
\end{aligned}
$$

Observe that $\mathcal{E}_{*}^{\prime}-\mathcal{E}_{*}>0 \Longrightarrow\left|\mathbf{v}_{*}^{\prime}\right|<\left|\mathbf{v}_{*}\right| \Longrightarrow$

$$
\mathcal{E}_{*}^{\prime}-\mathcal{E}_{*} \leq \alpha_{0} e^{-\beta_{0}\left|\mathbf{v}_{*}^{\prime}\right|} \beta_{0}\left(\left|\mathbf{v}_{*}\right|-\left|\mathbf{v}_{*}^{\prime}\right|\right) \leq \alpha_{0} \beta_{0} e^{-\beta_{0}\left|\mathbf{v}_{*}^{\prime}\right|}\left|\mathbf{v}^{\prime}-\mathbf{v}\right|
$$

This together with $\sqrt{1+|\mathbf{v}|^{2}+\left|\mathbf{v}_{*}\right|^{2}} \leq 1+\left|\mathbf{v}^{\prime}\right|+\left|\mathbf{v}_{*}^{\prime}\right|$ and $1+\frac{\beta_{0}}{2}\left|\mathbf{v}_{*}^{\prime}\right| \leq e^{\frac{\beta_{0}}{2}\left|\mathbf{v}_{*}^{\prime}\right|}$ gives

$$
\left(\mathcal{E}_{*}^{\prime}-\mathcal{E}_{*}\right)_{+} \sqrt{1+|\mathbf{v}|^{2}+\left|\mathbf{v}_{*}\right|^{2}} \leq C_{13}\left|\mathbf{v}^{\prime}-\mathbf{v}\right|\left\langle\mathbf{v}^{\prime}\right\rangle e^{-\frac{\beta_{0}}{2}\left|\mathbf{v}_{*}^{\prime}\right|} .
$$


Then using Lemma 4.1 we have

$$
\begin{aligned}
& \int_{\mathbb{R}^{3} \times \mathbb{R}^{3} \times \mathbb{S}^{2}}\left|\mathbf{v}-\mathbf{v}_{*}\right| f f^{\prime}\left(\mathcal{E}_{*}^{\prime}-\mathcal{E}_{*}\right)_{+} \sqrt{1+|\mathbf{v}|^{2}+\left|\mathbf{v}_{*}\right|^{2}} \mathrm{~d} \mathbf{v} \mathrm{d} \mathbf{v}_{*} \mathrm{~d} \sigma \\
& \leq C_{13} \int_{\mathbb{R}^{3}} f(\mathbf{v}, t)\left(\int_{\mathbb{R}^{3} \times \mathbb{S}^{2}}\left|\mathbf{v}-\mathbf{v}_{*}\right|\left|\mathbf{v}^{\prime}-\mathbf{v}\right|\left\langle\mathbf{v}^{\prime}\right\rangle f\left(\mathbf{v}^{\prime}, t\right) e^{-\frac{\beta_{0}}{2}\left|\mathbf{v}_{*}^{\prime}\right|} \mathrm{d} \mathbf{\mathbf { v } _ { * }} \mathrm{d} \sigma\right) \mathrm{d} \mathbf{v} \\
& \leq C_{14}\left(\|f(t)\|_{L_{1}^{1}}\right)^{2} \leq C_{14}\|f(t)\|_{L^{1}}\|f(t)\|_{L_{2}^{1}}
\end{aligned}
$$

and thus

$$
\frac{1}{4\left(1-e^{-t^{\delta}}\right)^{2}} \int_{\mathbb{R}^{3} \times \mathbb{R}^{3} \times \mathbb{S}^{2}} \bar{B}_{K} \cdot \Psi_{3} \log \left(\frac{G^{\prime} G_{*}^{\prime}}{G G_{*}}\right) \mathrm{d} \mathbf{v} \mathrm{d} \mathbf{v}_{*} \mathrm{~d} \sigma \leq C_{15} t^{\delta} e^{-t^{\delta}}\|f(t)\|_{L^{1}}\|f(t)\|_{L_{2}^{1}} .
$$

Combining all estimates (4.22), (4.23),(4.26), (4.27) (4.28), and recalling the conservation of mass and energy $\|f(t)\|_{L^{1}}=4 \pi \sqrt{2} N,\|f(t)\|_{L_{2}^{1}}=4 \pi \sqrt{2}(N+2 E)$ we obtain (4.13).

(II): In the following we will use $\sigma$-representation (4.5) for $\bar{B}_{K}\left(\mathbf{v}-\mathbf{v}_{*}, \sigma\right)$ and the bounds

$$
b_{0} \min \left\{1,\left|\mathbf{v}-\mathbf{v}_{*}\right|^{2 \eta}\right\} \leq \Phi\left(\left|\mathbf{v}-\mathbf{v}^{\prime}\right|,\left|\mathbf{v}-\mathbf{v}_{*}^{\prime}\right|\right) \leq 1
$$

which comes from the assumption (1.10). Let

$$
\mathcal{V}=\left\{\left(\mathbf{v}, \mathbf{v}_{*}, \sigma\right) \in \mathbb{R}^{3} \times \mathbb{R}^{3} \times \mathbb{S}^{2}\left|\Phi\left(\left|\mathbf{v}-\mathbf{v}^{\prime}\right|,\left|\mathbf{v}-\mathbf{v}_{*}^{\prime}\right|\right) \geq(4 \pi)^{2} K\right| \mathbf{v}-\mathbf{v}^{\prime}|| \mathbf{v}-\mathbf{v}_{*}^{\prime} \mid\right\}
$$

$\mathcal{V}^{c}=\mathbb{R}^{3} \times \mathbb{R}^{3} \times \mathbb{S}^{2} \backslash \mathcal{V}, q_{1}=p_{1} /\left(p_{1}-1\right), q_{2}=p_{2} /\left(p_{2}-1\right)$, and recall $\langle\mathbf{v}\rangle=\left(1+|\mathbf{v}|^{2}\right)^{1 / 2}$. Then using Hölder inequality we have

$$
\begin{aligned}
& \mathcal{D}_{2}(G(t))=\frac{1}{4} \int_{\mathbb{R}^{3} \times \mathbb{R}^{3} \times \mathbb{S}^{2}}\left\langle\mathbf{v}-\mathbf{v}_{*}\right\rangle^{2} \Gamma\left(G^{\prime} G_{*}^{\prime}, G G_{*}\right) \mathrm{d} \mathbf{v} \mathrm{d} \mathbf{v}_{*} \mathrm{~d} \sigma \\
& \leq \frac{1}{4}\left(\int_{\mathcal{V}} \frac{\left\langle\mathbf{v}-\mathbf{v}_{*}\right\rangle^{2 q_{1}}}{\left(\bar{B}_{K}\right)^{q_{1} / p_{1}}} \Gamma\left(G^{\prime} G_{*}^{\prime}, G G_{*}\right) \mathrm{d} \mathbf{v} \mathrm{d} \mathbf{v}_{*} \mathrm{~d} \sigma\right)^{1 / q_{1}}\left(\int_{\mathcal{V}} \bar{B}_{K} \Gamma\left(G^{\prime} G_{*}^{\prime}, G G_{*}\right) \mathrm{d} \mathbf{v} \mathrm{d} \mathbf{v}_{*} \mathrm{~d} \sigma\right)^{1 / p_{1}} \\
& +\frac{1}{4}\left(\int_{\mathcal{V}^{c}} \frac{\left\langle\mathbf{v}-\mathbf{v}_{*}\right\rangle^{2 q_{2}}}{\left(\bar{B}_{K}\right)^{q_{2} / p_{2}}} \Gamma\left(G^{\prime} G_{*}^{\prime}, G G_{*}\right) \mathrm{d} \mathbf{v} \mathrm{d} \mathbf{v}_{*} \mathrm{~d} \sigma\right)^{1 / q_{2}}\left(\int_{\mathcal{V}^{c}} \bar{B}_{K} \Gamma\left(G^{\prime} G_{*}^{\prime}, G G_{*}\right) \mathrm{d} \mathbf{v} \mathrm{d} \mathbf{v}_{*} \mathrm{~d} \sigma\right)^{1 / p_{2}} \\
& \leq\left(I_{q_{1}}(t)\right)^{1 / q_{1}}\left(D_{K}(F(t))^{1 / p_{1}}+\left(J_{q_{2}}(t)\right)^{1 / q_{2}}\left(D_{K}(F(t))^{1 / p_{2}}\right.\right.
\end{aligned}
$$

where we have used

$$
\frac{1}{4} \int_{\mathbb{R}^{3} \times \mathbb{R}^{3} \times \mathbb{S}^{2}} \bar{B}_{K}\left(\mathbf{v}-\mathbf{v}_{*}, \sigma\right) \Gamma\left(G^{\prime} G_{*}^{\prime}, G G_{*}\right) \mathrm{d} \mathbf{v} \mathrm{d} \mathbf{v}_{*} \mathrm{~d} \sigma \leq D_{K}(F(t)) .
$$

Let us compute using the first inequality in (4.21) that

$$
\begin{aligned}
I_{q_{1}}(t) & =\frac{1}{4} \int_{\frac{1}{(4 \pi)^{2}} \Phi\left(\left|\mathbf{v}-\mathbf{v}^{\prime}\right|,\left|\mathbf{v}-\mathbf{v}_{*}^{\prime}\right|\right) \geq K\left|\mathbf{v}-\mathbf{v}^{\prime}\right|\left|\mathbf{v}-\mathbf{v}_{*}^{\prime}\right|} \frac{\left\langle\mathbf{v}-\mathbf{v}_{*}\right\rangle^{2 q_{1}}}{\left(\bar{B}_{K}\left(\mathbf{v}-\mathbf{v}_{*}, \sigma\right)\right)^{q_{1} / p_{1}}} \Gamma\left(G^{\prime} G_{*}^{\prime}, G G_{*}\right) \mathrm{d} \mathbf{v} \mathrm{d} \mathbf{v}_{*} \mathrm{~d} \sigma \\
& \leq \frac{1}{2} \int_{\mathbb{R}^{3} \times \mathbb{R}^{3} \times \mathbb{S}^{2}} 1_{\left\{G G_{*}>G^{\prime} G_{*}^{\prime}\right\}} \frac{\left\langle\mathbf{v}-\mathbf{v}_{*}\right\rangle^{2 q_{1}}}{\left.\left[\frac{K}{2}\left|\mathbf{v}-\mathbf{v}_{*}\right|\left|\mathbf{v}-\mathbf{v}^{\prime}\right|\left|\mathbf{v}-\mathbf{v}_{*}^{\prime}\right|\right]\right]^{q_{1} / p_{1}}} \Gamma\left(G^{\prime} G_{*}^{\prime}, G G_{*}\right) \mathrm{d} \mathbf{v} \mathrm{d} \mathbf{v}_{*} \mathrm{~d} \sigma .
\end{aligned}
$$


By definition of $\Gamma(\cdot, \cdot)$ and using $(4.24)$ we have

$$
G G_{*}>G^{\prime} G_{*}^{\prime} \Longrightarrow \Gamma\left(G G_{*}, G^{\prime} G_{*}^{\prime}\right) \leq C_{16} t^{\delta} G G_{*} \sqrt{1+|\mathbf{v}|^{2}+\left|\mathbf{v}_{*}\right|^{2}} .
$$

Also we have

$$
\left|\mathbf{v}-\mathbf{v}^{\prime}\right|\left|\mathbf{v}-\mathbf{v}_{*}^{\prime}\right|=\frac{1}{2}\left|\mathbf{v}-\mathbf{v}_{*}\right|^{2} \sqrt{1-(\mathbf{n} \cdot \sigma)^{2}}, \quad \mathbf{n}=\frac{\mathbf{v}-\mathbf{v}_{*}}{\left|\mathbf{v}-\mathbf{v}_{*}\right|}
$$

and $\frac{q_{1}}{p_{1}}=q_{1}-1<1$. So

$$
\begin{aligned}
I_{q_{1}}(t) & \leq \frac{1}{2} C_{17} t^{\delta}\left(\frac{4}{K}\right)^{q_{1}-1} \int_{\mathbb{R}^{3} \times \mathbb{R}^{3} \times \mathbb{S}^{2}} \frac{\left\langle\mathbf{v}-\mathbf{v}_{*}\right\rangle^{2 q_{1}} G G_{*} \sqrt{1+|\mathbf{v}|^{2}+\left|\mathbf{v}_{*}\right|^{2}}}{\left[\left|\mathbf{v}-\mathbf{v}_{*}\right|^{3} \sqrt{1-(\mathbf{n} \cdot \sigma)^{2}}\right]^{q_{1}-1}} \mathrm{~d} \mathbf{v} \mathrm{d} \mathbf{v}_{*} \mathrm{~d} \sigma \\
& =C_{18} t^{\delta} K^{-\left(q_{1}-1\right)} \int_{\mathbb{R}^{3} \times \mathbb{R}^{3}} \frac{\left\langle\mathbf{v}-\mathbf{v}_{*}\right\rangle^{2 q_{1}} G G_{*} \sqrt{1+|\mathbf{v}|^{2}+\left|\mathbf{v}_{*}\right|^{2}}}{\left|\mathbf{v}-\mathbf{v}_{*}\right|^{3\left(q_{1}-1\right)}} \mathrm{d} \mathbf{v} \mathrm{d} \mathbf{v}_{*} .
\end{aligned}
$$

Next we have

$$
\begin{gathered}
\sqrt{1+|\mathbf{v}|^{2}+\left|\mathbf{v}_{*}\right|^{2}} \leq 2\left(\langle\mathbf{v}\rangle+\left\langle\mathbf{v}-\mathbf{v}_{*}\right\rangle\right) \leq 4\langle\mathbf{v}\rangle\left\langle\mathbf{v}-\mathbf{v}_{*}\right\rangle, \\
\int_{\mathbb{R}^{3}} \frac{\left\langle\mathbf{v}-\left.\mathbf{v}_{*}\right|^{2 q_{1}+1}\right.}{\left|\mathbf{v}-\mathbf{v}_{*}\right|^{3\left(q_{1}-1\right)}} G\left(\mathbf{v}_{*}, t\right) \mathrm{d} \mathbf{v}_{*} \leq C_{19}\left(1+\langle\mathbf{v}\rangle^{4-q_{1}}\|G(t)\|_{L_{4-q_{1}}^{1}}\right)
\end{gathered}
$$

where we have used $1<q_{1}<2$ and $0<G(\cdot)<1$. From (4.32), (4.33), and (4.34) we obtain

$$
I_{q_{1}}(t) \leq C_{20} t^{\delta} K^{-\left(q_{1}-1\right)}\left(1+\|G(t)\|_{L_{4-q_{1}}^{1}}\right)\|G(t)\|_{L_{5-q_{1}}^{1}} \leq C_{21} t^{\delta} K^{-\left(q_{1}-1\right)}, \quad t \geq 1
$$

where we used $0 \leq G(\cdot, t) \leq F(\cdot, t) \leq f(\cdot, t)+\mathcal{E}$ so that

$$
\|G(t)\|_{L_{s}^{1}} \leq\|f(t)\|_{L_{s}^{1}}+\|\mathcal{E}\|_{L_{s}^{1}} \leq C_{22} \quad \forall t \geq 1,0 \leq s \leq 4
$$

Next using (4.29), (4.31), the first inequality in (4.33), and the inequality $\Phi\left(\left|\mathbf{v}-\mathbf{v}^{\prime}\right|,\left|\mathbf{v}-\mathbf{v}_{*}^{\prime}\right|\right) \geq$ $b_{0} \min \left\{1,\left|\mathbf{v}-\mathbf{v}_{*}\right|^{2 \eta}\right\}$ we compute as the above that

$$
\begin{aligned}
& J_{q_{2}}(t)=\frac{1}{4} \int_{\frac{1}{(4 \pi)^{2}} \Phi\left(\left|\mathbf{v}-\mathbf{v}^{\prime}\right|,\left|\mathbf{v}-\mathbf{v}_{*}^{\prime}\right|\right)<K\left|\mathbf{v}-\mathbf{v}^{\prime}\right|\left|\mathbf{v}-\mathbf{v}_{*}^{\prime}\right|} \frac{\left\langle\mathbf{v}-\mathbf{v}_{*}\right\rangle^{2 q_{2}}}{\left(\bar{B}_{K}\left(\mathbf{v}-\mathbf{v}_{*}, \sigma\right)\right)^{q_{2} / p_{2}}} \Gamma\left(G^{\prime} G_{*}^{\prime}, G G_{*}\right) \mathrm{d} \mathbf{v} \mathrm{d} \mathbf{v}_{*} \mathrm{~d} \sigma \\
& \leq C_{23} \int_{\mathbb{R}^{3} \times \mathbb{R}^{3} \times \mathbb{S}^{2}} \frac{\left\langle\mathbf{v}-\mathbf{v}_{*}\right\rangle^{2 q_{2}}}{\left(\left|\mathbf{v}-\mathbf{v}_{*}\right| \min \left\{1,\left|\mathbf{v}-\mathbf{v}_{*}\right|^{2 \eta}\right\}\right)^{q_{2} / p_{2}}} \Gamma\left(G^{\prime} G_{*}^{\prime}, G G_{*}\right) \mathrm{d} \mathbf{v} \mathrm{d} \mathbf{v}_{*} \mathrm{~d} \sigma \\
& =2 C_{23} \int_{\mathbb{R}^{3} \times \mathbb{R}^{3} \times \mathbb{S}^{2}, G G_{*}>G^{\prime} G_{*}^{\prime}} \frac{\left\langle\mathbf{v}-\mathbf{v}_{*}\right\rangle^{2 q_{2}}}{\left(\left|\mathbf{v}-\mathbf{v}_{*}\right| \min \left\{1,\left.\left|\mathbf{v}-\mathbf{v}_{*}\right|\right|^{2 \eta}\right\}\right)^{q_{2} / p_{2}}} \Gamma\left(G^{\prime} G_{*}^{\prime}, G G_{*}\right) \mathrm{d} \mathbf{v} \mathrm{d} \mathbf{v}_{*} \mathrm{~d} \sigma \\
& \leq C_{24} t^{\delta} \int_{\mathbb{R}^{3} \times \mathbb{R}^{3} \times \mathbb{S}^{2}} \frac{\left\langle\mathbf{v}-\mathbf{v}_{*}\right\rangle^{2 q_{2}}\left(\langle\mathbf{v}\rangle+\left\langle\mathbf{v}-\mathbf{v}_{*}\right\rangle\right)}{\left(\left|\mathbf{v}-\mathbf{v}_{*}\right| \min \left\{1,\left|\mathbf{v}-\mathbf{v}_{*}\right|^{2 \eta}\right\}\right)^{q_{2} / p_{2}}} G G_{*} \mathrm{~d} \mathbf{v} \mathrm{d} \mathbf{v}_{*} \mathrm{~d} \sigma \\
& \leq C_{25} t^{\delta}\left(\|G(t)\|_{L_{1}^{1}}+\|G(t)\|_{L^{1}}\|G(t)\|_{L_{2+q_{2}}^{1}}\right) \leq C_{26} t^{\delta}, \quad t \geq 1
\end{aligned}
$$

where we have used the condition $(1+2 \eta) \frac{q_{2}}{p_{2}}<3$ and $0<G(\cdot)<1$. Inserting (4.35), (4.36) into (4.30) and using $\frac{q_{1}-1}{q_{1}}=\frac{1}{p_{1}}$ we obtain (4.14). 
(III): Let $B_{\min }\left(\mathbf{v}-\mathbf{v}_{*}, \omega\right)$ be given by $(2.8)$ with $\Psi_{0}(r)=\min \left\{1, r^{1+2 \eta}\right\}$, and let $D_{\min }(\cdot)$ be the corresponding entropy dissipation. By Assumption 1.1 and (4.9), it is easily seen that $B_{\min }\left(\mathbf{v}-\mathbf{v}_{*}, \omega\right) \leq B_{K}\left(\mathbf{v}-\mathbf{v}_{*}, \omega\right)$ so that $D_{\min }(\cdot) \leq D_{K}(\cdot)$ for all $K \geq 1$.

From (4.16) and the lower and upper bounds (4.17), (4.18) we see that Proposition 2.2 applies to $F(\cdot, t)=F^{K}(\cdot, t), G(\cdot, t)=G^{K}(\cdot, t)$ (for any $t \geq 1, K \in \mathbb{N}$ ) so that

$$
S(\Omega)-S(F(t))) \leq C_{27}\left(\left(\mathcal{D}_{2}(G(t))\right)^{\frac{1}{10}}+\left(D_{\min }(F(t))\right)^{\frac{1}{4}}\right) .
$$

For any $1<T<\infty$ and any $K \geq T^{\delta(p-2)}$ we have from part (II) that for any $t \in[1, T]$

$$
\left(\mathcal{D}_{2}(G(t))\right)^{\frac{1}{10}} \leq\left(C_{2}\right)^{\frac{1}{10}}\left(\left(t^{\delta} D_{K}(F(t))\right)^{\frac{1}{10 p_{1}}}+\left(t^{\delta} D_{K}(F(t))\right)^{\frac{1}{10 p_{2}}}\right)
$$

hence from $(4.37)$ and $D_{\min }(\cdot) \leq D_{K}(\cdot)$ we obtain

$$
S(\Omega)-S(F(t)) \leq C_{3}\left(\left(t^{\delta} D_{K}(F(t))\right)^{\frac{1}{10\left(p_{1} \vee p_{2}\right)}}+\left(t^{\delta} D_{K}(F(t))\right)^{\frac{1}{4}}\right)=\Psi\left(t^{\delta} D_{K}(F(t))\right)
$$

that is,

$$
t^{-\delta} \Psi^{-1}(S(\Omega)-S(F(t))) \leq D_{K}(F(t)), \quad t \in[1, T]
$$

Inserting this inequality into (4.13) in part (I) gives (4.15). The proof is complete.

The next lemma deals with a differential inequality that implies an algebraic rate of decay.

Lemma 4.3. Let $a>0, b>0, \alpha>0,0<\beta<1,0<\gamma<1, \delta<1, k \geq 0$ and $u_{0} \geq 0$ be finite constants, $\Psi(y)=a\left(y^{\beta}+y^{\gamma}\right), y \in[0, \infty)$, and let $\Psi^{-1}(u), u \in[0, \infty)$, be the inverse function of $\Psi(y)$. Given any $T>1$. Assume that $t \mapsto u(t) \in[0, \infty)$ is absolutely continuous on $[1, T]$ satisfying $u(1) \leq u_{0}$ and

$$
\frac{\mathrm{d}}{\mathrm{d} t} u(t) \leq-t^{-\delta} \Psi^{-1}(u(t))+b t^{k} e^{-t^{\alpha}} \quad \text { a.e. } \quad t \in[1, T] .
$$

Then

$$
u(t) \leq C t^{-\lambda} \quad \forall t \in[1, T]
$$

where $\lambda=\frac{\beta \wedge \gamma}{1-(\beta \wedge \gamma)}(1-\delta)$ and $0<C<\infty$ depends only on $a, b, \alpha, \beta, \gamma, \delta, k$ and $u_{0}$. In particular $C$ does not depend on $T$.

Proof. Let

$$
m^{*}(\beta)=\max _{t \geq 1} t^{\beta(k+\delta)+\lambda} e^{-\beta t^{\alpha}}, \quad m^{*}(\gamma)=\max _{t \geq 1} t^{\gamma(k+\delta)+\lambda} e^{-\gamma t^{\alpha}}
$$

and choose a least constant $C>0$ such that $C \geq u_{0}$ and

$$
\frac{\lambda^{\beta}}{C^{1-\beta}}+\frac{\lambda^{\gamma}}{C^{1-\gamma}}+\frac{b^{\beta} m^{*}(\beta)}{C}+\frac{b^{\gamma} m^{*}(\gamma)}{C} \leq \frac{1}{a}
$$

Let $U(t)=C t^{-\lambda}$. It is easily checked that (4.38) and $\lambda=\frac{\beta \vee \gamma}{1-(\beta \vee \gamma)}(1-\delta)>0$ imply

$$
\frac{\mathrm{d}}{\mathrm{d} t} U(t)+t^{-\delta} \Psi^{-1}(U(t))-b t^{k} e^{-t^{\alpha}} \geq 0 \quad \forall t \geq 1
$$


From this and $u(1) \leq U(1)$ and that $u(t)$ is absolutely continuous on $[1, T]$, we have

$$
\begin{aligned}
& (u(t)-U(t))_{+}=\int_{1}^{t}\left(\frac{\mathrm{d}}{\mathrm{d} \tau} u(\tau)-\frac{\mathrm{d}}{\mathrm{d} \tau} U(\tau)\right) 1_{\{u(\tau)>U(\tau)\}} \mathrm{d} \tau \\
& \leq \int_{1}^{t}\left(-\tau^{-\delta} \Psi^{-1}(u(\tau))+b \tau^{k} e^{-\tau^{\alpha}}-\frac{\mathrm{d}}{\mathrm{d} \tau} U(\tau)\right) 1_{\{u(\tau)>U(\tau)\}} \mathrm{d} \tau \\
& \leq \int_{1}^{t}\left(-\tau^{-\delta} \Psi^{-1}(U(\tau))+b \tau^{k} e^{-\tau^{\alpha}}-\frac{\mathrm{d}}{\mathrm{d} \tau} U(\tau)\right) 1_{\{u(\tau)>U(\tau)\}} \mathrm{d} \tau \leq 0 \quad \forall t \in[1, T] .
\end{aligned}
$$

Here we used the increase of $u \mapsto \Psi^{-1}(u)$ on $[0, \infty)$. Thus $u(t) \leq U(t)$ for all $t \in[1, T]$.

Finally we can state and prove the main result of this section:

Theorem 4.4. Let $B\left(\mathbf{v}-\mathbf{v}_{*}, \omega\right)$ satisfy Assumption 1.1, let $F_{0} \in \mathcal{B}_{1}^{+}\left(\mathbb{R}_{\geq 0}\right)$ satisfy $N\left(F_{0}\right)>$ $0, E\left(F_{0}\right)>0$, and let $F_{\text {be }}$ be the unique Bose-Einstein distribution with the same mass $N=$ $N\left(F_{0}\right)$ and energy $E=E\left(F_{0}\right)$. Let $F_{t}$ be a conservative measure-valued isotropic solution $F_{t}$ obtained in Proposition 3.4 with the initial datum $F_{0}$. Then $S\left(F_{t}\right) \geq S\left(F_{0}\right), S\left(F_{t}\right)>0$ for all $t>0$, and for any $0<\lambda<\frac{1}{10 \max \left\{2, \frac{4+2 \eta}{3}\right\}-1}$ we have

$$
0 \leq S\left(F_{\text {be }}\right)-S\left(F_{t}\right) \leq C(1+t)^{-\lambda} \quad \forall t \geq 0
$$

where $C \in(0, \infty)$ depends only on $N, E, b_{0}, \eta$ and $\lambda$.

Proof. Let $\lambda_{n}=\frac{1-\frac{1}{n}}{10 \max \left\{2+\frac{1}{n}, \frac{4+2 \eta}{3}+\frac{1}{n}\right\}-1}, n=1,2,3, \ldots$. Then $\lambda_{n} \rightarrow \frac{1}{10 \max \left\{2, \frac{4+2 \eta}{3}\right\}-1}>\lambda(n \rightarrow$ $\infty)$. So we can find a smallest $n_{\lambda} \in \mathbb{N}$, which depends only on $\eta$ and $\lambda$, such that $\lambda \leq \lambda_{n_{\lambda}}$. Let $p_{1}=2+\frac{1}{n_{\lambda}}, p_{2}=\frac{4+2 \eta}{3}+\frac{1}{n_{\lambda}}, \delta=\frac{1}{n_{\lambda}}$.

As mentioned in the theorem, $F_{t}$ is a weak limit of a subsequence $f^{K_{n}}(\cdot, t)$ of $f^{K}(\cdot, t)$ which are the isotropic approximate solutions of Eq.(1.1) on $\mathbb{R}^{3} \times[0, \infty)$ obtained in Proposition 3.4 with the initial data $f_{0}^{K}$ which together with $f^{K}$ have all properties in part (I) and part (II) of Proposition 3.4. Since $t_{0}$ in (3.16) is arbitrary, this implies that $S\left(F_{t}\right)>0$ for all $t>0$. We also recall part (I) of Lemma 2.1 that ensures $S\left(F_{t}\right) \leq S\left(F_{\text {be }}\right)$ for all $t \in[0, \infty)$. From (3.13) and (3.15) and taking $t_{0}=1$ we have

$$
\inf _{K \in \mathbb{N}, t \geq 1} S\left(f^{K}(t) \geq S_{0}:=S_{*}(1)>0, \quad \sup _{K \in \mathbb{N}, t \geq 1}\left\|f^{K}(t)\right\|_{L_{2+2 q_{2}}^{1}} \leq C_{0}<\infty\right.
$$

where $S_{0}$ depends only on $N, E, b_{0}$ and $\eta ; q_{2}=p_{2} /\left(p_{2}-1\right)$, and $C_{0}$ depends only on $N, E, b_{0}, \eta$ and $\lambda$. Now $f_{0}^{K}, f^{K}$ satisfy all assumptions in Proposition 4.2 and, for the present case, the constants $C_{1}, C_{2}, \ldots, C_{27}$ in the proof of Proposition 4.2 and thus the constants $C_{28}, C_{29}, C_{30} \in(0, \infty)$ appeared below, all depend only on $N, E, b_{0}, \eta$ and $\lambda$. Let $F^{K}(\mathbf{v}, t)=\left(1-e^{-t^{\delta}}\right) f^{K}\left(|\mathbf{v}|^{2} / 2, t\right)+$ $e^{-t^{\delta}} \mathcal{E}(\mathbf{v})$ be defined in Proposition 4.2. Then with the notations in Proposition 4.2 and applying Lemma 4.3 to part (III) of Proposition 4.2 with functions $0 \leq u(t)=S(\Omega)-S\left(F^{K}(t)\right) \leq S(\Omega)$ and $\Psi(y)=C_{3}\left(y^{\frac{1}{10 p_{1} \vee p_{2}}}+y^{\frac{1}{4}}\right)$ we have

$$
S(\Omega)-S\left(F^{K}(t)\right) \leq C_{28} t^{-\lambda^{*}} \leq C_{28} t^{-\lambda}, \quad \forall t \in[1, T]
$$


where $\lambda^{*}=\lambda_{n_{\lambda}} \geq \lambda$. On the other hand, from (1.29), (1.27) we have

$$
S\left(F^{K}(t)\right) \leq S\left(\left(1-e^{-t^{\delta}}\right) f^{K}(t)\right)+S\left(e^{-t^{\delta}} \mathcal{E}\right) \leq S\left(f^{K}(t)\right)+C_{29} t^{\delta} e^{-t^{\delta}} \leq \quad \forall t \geq 1
$$

Thus, for any $T>1$,

$$
S(\Omega)-S\left(f^{K}(t)\right) \leq C_{28} t^{-\lambda}+C_{29} t^{\delta} e^{-t^{\delta}} \leq C_{30} t^{-\lambda} \quad \forall t \in[1, T], \forall K \geq T^{\delta(p-2)} .
$$

Also, from the convergence (3.18), (3.20), the non-decrease of $t \mapsto S\left(f^{K}(t)\right)$ (see (3.4)), the definition of entropy $S\left(\bar{F}_{t}\right)$, and $S\left(\bar{F}_{t}\right)=S\left(F_{t}\right)$, we have

$$
S\left(F_{0}\right)=\lim _{n \rightarrow \infty} S\left(f_{0}^{K_{n}}\right)=\limsup _{n \rightarrow \infty} S\left(f^{K_{n}}(0)\right) \leq \limsup _{n \rightarrow \infty} S\left(f^{K_{n}}(t)\right) \leq S\left(\bar{F}_{t}\right)=S\left(F_{t}\right)
$$

for all $t \in[0, \infty)$. Thus for any $T>1$, applying (4.40) to $f^{K_{n}}(\cdot, t)$ with $K_{n}>T^{\delta(p-2)}$, we obtain (because $S\left(F_{\text {be }}\right)=S(\Omega)$ )

$$
S\left(F_{\text {be }}\right)-S\left(F_{t}\right) \leq \limsup _{n \rightarrow \infty}\left(S(\Omega)-S\left(f^{K_{n}}(t)\right)\right) \leq C_{30} t^{-\lambda} \quad \forall t \in[1, T] .
$$

Since $C_{30}$ is independent of $T$ and $T$ can be arbitrarily large, we conclude

$$
0 \leq S\left(F_{\mathrm{be}}\right)-S\left(F_{t}\right) \leq C_{30} t^{-\lambda} \quad \forall t \in[1, \infty)
$$

This gives (4.39) and finishes the proof of the theorem.

\section{Rate of Convergence to BEC}

In this section we prove the second part of the main result Theorem 1.4: an algebraic rate of convergence of $F_{t}(\{0\})$ to the Bose-Einstein condensation $F_{\text {be }}(\{0\})=\left(1-\left(\bar{T} / \bar{T}_{c}\right)^{3 / 5}\right) N$. The completion of the proof of Theorem 1.4 is given at the end of this section. We first prove some properties of the collision integrals that have been proven to hold for the hard sphere model, then we present our new progress on proving lower bounds of condensation $F_{t}(\{0\})$ without any additional assumption on the initial data $F_{0}$.

The monotone assumption (1.9) is now used to prove the following

Proposition 5.1. (Convex-Positivity). Let $W(x, y, z), \mathcal{K}[\varphi](x, y, z)$ be defined in (1.17)(1.21) with $\Phi$ satisfying (1.5),(1.9). Then for any convex function $\varphi \in C_{b}^{1,1}\left(\mathbb{R}_{\geq 0}\right)$ we have

$$
\int_{\mathbb{R}_{\geq 0}^{3}} \mathcal{K}[\varphi] \mathrm{d}^{3} F \geq \int_{\mathbb{R}_{\geq 0}^{3}} \mathcal{K}_{1}[\varphi] \mathrm{d}^{3} F+\int_{\mathbb{R}_{>0}^{3}} \mathcal{K}_{2}[\varphi] \mathrm{d}^{3} F \geq 0
$$

where

$$
\begin{aligned}
& \mathcal{K}_{1}[\varphi](x, y, z)=1_{\{0 \leq x<y \leq z\}} \chi_{y, z} W(x, y, z) \Delta_{\mathrm{sym}} \varphi(x, y, z) \geq 0, \\
& \mathcal{K}_{2}[\varphi](x, y, z)=1_{\{0<y \leq z<x<y+z\}} \chi_{y, z} W(x, y, z) \Delta \varphi(x, y, z) \geq 0, \\
& \chi_{y, z}=\left\{\begin{array}{lll}
2 & \text { if } y<z, \\
1 & \text { if } \quad y=z
\end{array}\right.
\end{aligned}
$$


for all $(x, y, z) \in \mathbb{R}_{\geq 0}^{3}, \Delta \varphi(x, y, z)$ is given in (1.18), and

$$
\begin{aligned}
& \Delta_{\text {sym }} \varphi(x, y, z)=\varphi(z+y-x)+\varphi(z+x-y)-2 \varphi(z) \\
& =(y-x)^{2} \int_{0}^{1} \int_{0}^{1} \varphi^{\prime \prime}(z+(s-t)(y-x)) \mathrm{d} s \mathrm{~d} t, \quad 0 \leq x, y \leq z .
\end{aligned}
$$

Proof. The positivity in (5.2),(5.3) follows from the convexity of $\varphi$ and (5.5), (1.18). To prove the inequality (5.1), we first use the symmetry $W(x, y, z)=W(x, z, y)$ on $\mathbb{R}_{\geq 0}^{3}$ (see Remark 6.4 in Appendix) and (1.18) to get

$$
\mathcal{K}[\varphi](x, y, z)=\mathcal{K}[\varphi](x, z, y),\left.\quad \mathcal{K}[\varphi](x, y, z)\right|_{x=y}=\left.\mathcal{K}[\varphi](x, y, z)\right|_{x=z}=0
$$

and then we make a decomposition according to $x$ lies in the left side, between, and right side of $y$ and $z$ respectively (using (5.6) and recalling that $\mathrm{d}^{3} F=\mathrm{d} F(x) \mathrm{d} F(y) \mathrm{d} F(z)$ ):

$$
\begin{aligned}
\int_{\mathbb{R}_{\geq 0}^{3}} \mathcal{K}[\varphi] \mathrm{d}^{3} F & =\left(2 \int_{0 \leq x<y<z}+2 \int_{0 \leq y<x<z}+\int_{0 \leq x<y=z}+\int_{0 \leq y, z<x}\right) W(x, y, z) \Delta \varphi(x, y, z) \mathrm{d}^{3} F \\
& :=I_{1}+I_{2}+I_{3}+I_{4} .
\end{aligned}
$$

After exchanging notations $x \leftrightarrow y$ for the integrand in $I_{2}$ we have

$$
\begin{aligned}
I_{1}+I_{2} & =2 \int_{0 \leq x<y<z} W(x, y, z) \Delta_{\mathrm{sym}} \varphi(x, y, z) \mathrm{d}^{3} F \\
& +2 \int_{0 \leq x<y<z}(W(y, x, z)-W(x, y, z)) \Delta \varphi(y, x, z) \mathrm{d}^{3} F
\end{aligned}
$$

where we used $\Delta \varphi(x, y, z)+\Delta \varphi(y, x, z)=\Delta_{\operatorname{sym}} \varphi(x, y, z)$ for $0 \leq x, y \leq z$. We need to prove

$$
(W(y, x, z)-W(x, y, z)) \Delta \varphi(y, x, z) \geq 0 \quad \forall 0 \leq x<y<z .
$$

By convexity of $\varphi$ and (1.18) we have

$$
\Delta \varphi(y, x, z)=\varphi(y)+\varphi(x+z-y)-\varphi(x)-\varphi(z) \leq 0 \quad \forall 0 \leq x<y \leq z .
$$

Therefore to prove (5.8) we need only prove

$$
W(y, x, z) \leq W(x, y, z) \quad \forall 0 \leq x<y \leq z .
$$

For any $0<x<y \leq z$ we have

$$
|\sqrt{x}-\sqrt{y}| \vee\left|\sqrt{x_{*}}-\sqrt{z}\right|=|\sqrt{x}-\sqrt{y}|, \quad(\sqrt{x}+\sqrt{y}) \wedge\left(\sqrt{x_{*}}+\sqrt{z}\right)=\sqrt{x}+\sqrt{y}
$$

hence

$$
\begin{aligned}
& W(x, y, z)=\frac{1}{4 \pi \sqrt{x y z}} \int_{|\sqrt{x}-\sqrt{y}|}^{\sqrt{x}+\sqrt{y}} \mathrm{~d} s \int_{0}^{2 \pi} \Phi\left(\sqrt{2} s, \sqrt{2} Y_{*}\right) \mathrm{d} \theta, \\
& W(y, x, z)=\frac{1}{4 \pi \sqrt{x y z}} \int_{|\sqrt{y}-\sqrt{x}|}^{\sqrt{y}+\sqrt{x}} \mathrm{~d} s \int_{0}^{2 \pi} \Phi\left(\sqrt{2} s, \sqrt{2} Y_{*}^{\sharp}\right) \mathrm{d} \theta
\end{aligned}
$$


where $Y_{*}=Y_{*}(x, y, z, s, \theta)$ is defined in (1.21) and $Y_{*}^{\sharp}=Y_{*}(y, x, z, s, \theta)$. By calculation (with the relation (6.6) in Appendix) it is easily checked that

$$
Y_{*}^{\sharp} \leq Y_{*} \quad \forall s \in[\sqrt{y}-\sqrt{x}, \sqrt{y}+\sqrt{x}] \quad(0<x<y \leq z) .
$$

Since $\rho \rightarrow \Phi(r, \rho)$ is non-decreasing, it follows that $W(y, x, z) \leq W(x, y, z)$. For the case $0=x<y \leq z$, we have $\Phi(\sqrt{2 y}, \sqrt{2(z-y)}) \leq \Phi(\sqrt{2 y}, \sqrt{2 z})$ which means (by definition of $W$ ) that the inequality $W(y, 0, z) \leq W(0, y, z)$ holds also true. This proves (5.9).

Now from $(5.7),(5.8)$ and $\left.\Delta \varphi(x, y, z)\right|_{y=z}=\left.\Delta_{\mathrm{sym}} \varphi(x, y, z)\right|_{y=z}$, and recalling definition of $\chi_{y, z}$ we obtain

$$
\begin{aligned}
& I_{1}+I_{2}+I_{3} \geq 2 \int_{0 \leq x<y<z} W(x, y, z) \Delta_{\text {sym }} \varphi(x, y, z) \mathrm{d}^{3} F+I_{3} \\
& =\int_{0 \leq x<y \leq z} \chi_{y, z} W(x, y, z) \Delta_{\text {sym }} \varphi(x, y, z) \mathrm{d}^{3} F=\int_{\mathbb{R}_{\geq 0}^{3}} \mathcal{K}_{1}[\varphi] \mathrm{d}^{3} F .
\end{aligned}
$$

For the last term $I_{4}$, observe that $0 \leq y, z<x$ and $W(x, y, z)>0$ imply $y>0, z>0$ and $x<y+z$. Thus

$$
I_{4}=\int_{0<y, z<x} W(x, y, z) \Delta \varphi(x, y, z) \mathrm{d}^{3} F=\int_{\mathbb{R}_{>0}^{3}} \mathcal{K}_{2}[\varphi] \mathrm{d}^{3} F
$$

Notation. To study local behavior of a measure $F \in \mathcal{B}^{+}\left(\mathbb{R}_{\geq 0}\right)$ near the origin, we introduce the following integrals. For $p>0, \varepsilon>0, \alpha \geq 0$, define

$$
\begin{aligned}
& N_{0, p}(F, \varepsilon)=\int_{\mathbb{R}_{\geq 0}}\left[\left(1-\frac{x}{\varepsilon}\right)_{+}\right]^{p} \mathrm{~d} F(x)=\int_{[0, \varepsilon]}\left(1-\frac{x}{\varepsilon}\right)^{p} \mathrm{~d} F(x), \\
& N_{\alpha, p}(F, \varepsilon)=\frac{1}{\varepsilon^{\alpha}} N_{0, p}(F, \varepsilon), \quad \underline{N}_{\alpha, p}(F, \varepsilon)=\inf _{0<\delta \leq \varepsilon} N_{\alpha, p}(F, \delta), \\
& A_{0, p}(F, \varepsilon)=\int_{[0, \varepsilon]}\left(\frac{x}{\varepsilon}\right)^{p} \mathrm{~d} F(x), \quad A_{\alpha, p}(F, \varepsilon)=\frac{1}{\varepsilon^{\alpha}} \int_{[0, \varepsilon]}\left(\frac{x}{\varepsilon}\right)^{p} \mathrm{~d} F(x) .
\end{aligned}
$$

Lemma 5.2. Let $B\left(\mathbf{v}-\mathbf{v}_{*}, \omega\right)$ be given by (1.4), (1.5), (1.9) where $\Phi$ also satisfies $0 \leq \Phi \leq 1$ on $\mathbb{R}_{\geq 0}^{2}$. Let $F_{t} \in \mathcal{B}_{1}^{+}\left(\mathbb{R}_{\geq 0}\right)$ be a conservative measure-valued isotropic solution of Eq.(1.1) on $[0, \infty)$ with the initial datum $F_{0}$ satisfying $N=N\left(F_{0}\right)>0, E=E\left(F_{0}\right)>0$. Then with $c=\sqrt{N E}$ we have:

(I) For any convex function $0 \leq \varphi \in C_{b}^{1,1}\left(\mathbb{R}_{\geq 0}\right)$

$$
e^{c t} \int_{\mathbb{R}_{\geq 0}} \varphi \mathrm{d} F_{t}-e^{c s} \int_{\mathbb{R}_{\geq 0}} \varphi \mathrm{d} F_{s} \geq \int_{s}^{t} e^{c \tau} \mathrm{d} \tau \int_{\mathbb{R}_{\geq 0}^{3}} \mathcal{K}[\varphi] \mathrm{d}^{3} F_{\tau} \geq 0 \quad \forall 0 \leq s<t .
$$

(II) For any convex function $0 \leq \varphi \in C_{b}\left(\mathbb{R}_{\geq 0}\right)$, the function $t \mapsto e^{c t} \int_{\mathbb{R}_{\geq 0}} \varphi \mathrm{d} F_{t}$ is nondecreasing on $[0, \infty)$, and thus for any $p \geq 1, \varepsilon>0, \alpha>0$, the functions $t \mapsto e^{\text {ct }} N_{0, p}\left(F_{t}, \varepsilon\right)$, $t \mapsto e^{c t} \underline{N}_{\alpha, p}\left(F_{t}, \varepsilon\right)$, and $t \mapsto e^{c t} F_{t}(\{0\})$ are all non-decreasing on $[0, \infty)$. 
Proof. (I): We first prove that

$$
\mathcal{J}[\varphi](y, z) \geq-\frac{1}{2} \varphi(y) \sqrt{z}-\frac{1}{2} \varphi(z) \sqrt{y}
$$

Since $\varphi \in C_{b}^{1,1}\left(\mathbb{R}_{\geq 0}\right)$ is convex, it is easily seen that $\varphi$ is non-increasing on $\mathbb{R}_{\geq 0}$. Given any $y, z \geq 0$. By symmetry $\mathcal{J}[\varphi](y, z)=\mathcal{J}[\varphi](z, y)$ we may assume that $y \leq z$. By (1.18) we have $\Delta \varphi(x, y, z) \geq 0$ for all $x \in[0, y] \cup[z, y+z]$ and so $\mathcal{J}[\varphi] \geq \frac{1}{2} \int_{y}^{z} \sqrt{x} W(x, y, z) \Delta \varphi(x, y, z) \mathrm{d} x$. To prove (5.12) we can assume that $y<z$. By the assumption on $\Phi$, the definition of $W(x, y, z)$, and that $\varphi$ is non-negative and non-increasing, we deduce $0 \leq W(x, y, z) \leq 1 / \sqrt{x z}$ and $\Delta \varphi(x, y, z) \geq-\varphi(y)$ for all $x \in(y, z)$. Thus

$$
\mathcal{J}[\varphi] \geq-\frac{1}{2 \sqrt{z}} \int_{y}^{z} \Delta \varphi(x, y, z) d x \geq-\frac{1}{2 \sqrt{z}} \varphi(y)(z-y) \geq-\frac{1}{2} \varphi(y) \sqrt{z}
$$

and so (5.12) holds true.

From (5.12) we have

$$
\int_{\mathbb{R}_{\geq 0}^{2}} \mathcal{J}[\varphi] \mathrm{d}^{2} F_{t} \geq-M_{1 / 2}\left(F_{t}\right) \int_{\mathbb{R}_{\geq 0}} \varphi \mathrm{d} F_{t} \geq-c \int_{\mathbb{R}_{\geq 0}} \varphi \mathrm{d} F_{t}
$$

where we used Cauchy-Schwarz inequality and the conservation of mass and energy to get $M_{1 / 2}\left(F_{t}\right) \leq \sqrt{N\left(F_{t}\right) E\left(F_{t}\right)}=\sqrt{N E}=c$. So by definition of measure-valued isotropic solutions we obtain

$$
\frac{\mathrm{d}}{\mathrm{d} t} \int_{\mathbb{R}_{\geq 0}} \varphi \mathrm{d} F_{t} \geq-c \int_{\mathbb{R}_{\geq 0}} \varphi \mathrm{d} F_{t}+\int_{\mathbb{R}_{\geq 0}^{3}} \mathcal{K}[\varphi] \mathrm{d}^{3} F_{t} \quad \forall t \in[0, \infty)
$$

i.e.

$$
\frac{\mathrm{d}}{\mathrm{d} t}\left(e^{c t} \int_{\mathbb{R}_{\geq 0}} \varphi \mathrm{d} F_{t}\right) \geq e^{c t} \int_{\mathbb{R}_{\geq 0}^{3}} \mathcal{K}[\varphi] \mathrm{d}^{3} F_{t} \geq 0 \quad \forall t \in[0, \infty) .
$$

This implies (5.11), here " $\geq 0$ " is due to the convex-positivity (Proposition 5.1).

(II): From (I) we see that for any convex function $0 \leq \varphi \in C_{b}^{1,1}\left(\mathbb{R}_{\geq 0}\right)$, the function $t \mapsto$ $e^{c t} \int_{\mathbb{R}_{\geq 0}} \varphi \mathrm{d} F_{t}$ is non-decreasing on $[0, \infty)$. Using approximation as did in [22], this monotone property holds also for any convex function $0 \leq \varphi \in C_{b}\left(\mathbb{R}_{\geq 0}\right)$. Applying this to the convex function $\varphi(x)=\left[(1-x / \varepsilon)_{+}\right]^{p}(\varepsilon>0, p \geq 1)$ we deduce that $e^{c t} N_{0, p}\left(F_{t}, \varepsilon\right), e^{c t} N_{\alpha, p}\left(F_{t}, \varepsilon\right)$, and thus $e^{c t} \underline{N}_{\alpha, p}\left(F_{t}, \varepsilon\right)$ are all non-decreasing on $t \in[0, \infty)$. Since $F_{t}(\{0\})=\lim _{\varepsilon \rightarrow 0+} N_{0, p}\left(F_{t}, \varepsilon\right)$ for all $t \geq 0$, it follows that $t \mapsto e^{c t} F_{t}(\{0\})$ is also non-decreasing on $[0, \infty)$.

Lemma 5.3. Let $\mathcal{J}[\varphi], \mathcal{K}[\varphi]$ be defined in (1.17)-(1.21) where $\Phi(r, \rho)$ satisfies Assumption 1.1 with $0 \leq \eta \leq 1 / 2$. Let $F \in \mathcal{B}^{+}\left(\mathbb{R}_{\geq 0}\right), \varphi_{\varepsilon}(x)=\left[(1-x / \varepsilon)_{+}\right]^{2}, 0<\varepsilon \leq 1,0 \leq \alpha<1-\eta$. Then

$$
\begin{aligned}
& \int_{\mathbb{R}_{\geq 0}^{2}} \mathcal{J}\left[\varphi_{\varepsilon}\right] \mathrm{d}^{2} F \geq \frac{b_{0}}{34} \varepsilon^{3 / 2}\left(\int_{\left[\frac{1}{2} \varepsilon, 1\right]} y^{-\frac{1-\eta}{2}} \mathrm{~d} F(y)\right)^{2}-2 M_{1 / 2}(F) N_{0,2}(F, \varepsilon), \\
& \int_{\mathbb{R}_{\geq 0}^{3}} \mathcal{K}\left[\varphi_{\varepsilon}\right] \mathrm{d}^{3} F \geq \frac{b_{0}}{2} \underline{N}_{\alpha, 2}(F, \varepsilon)\left(A_{\beta, p}(F, \varepsilon)\right)^{2}
\end{aligned}
$$


where $p=\frac{3}{2}+\alpha, \beta=\frac{1-\alpha-\eta}{2}$. Also if $0<\varepsilon \leq 2 / 3$ and $0<\gamma \leq(24)^{-2 / 3}$, then

$$
\int_{\mathbb{R}_{\geq 0}^{3}} \mathcal{K}\left[\varphi_{\varepsilon}\right] \mathrm{d}^{3} F \geq \frac{b_{0}}{4} \frac{\gamma^{3 / 2}}{\varepsilon^{1-\eta}} N_{0,2}(F, \gamma \varepsilon)\left(F\left(\left[\gamma \varepsilon, \frac{3}{2} \varepsilon\right]\right)\right)^{2} .
$$

Proof. We first prove that

$$
W(x, y, z) \geq \frac{b_{0}}{2} \frac{z^{\eta}}{\sqrt{y z}} \quad \forall 0 \leq x<y \leq z \leq 1 .
$$

First of all from the assumption on $\Phi$ we have (see (1.10))

$$
\Phi(r, \rho) \geq b_{0} \min \left\{1,\left(r^{2}+\rho^{2}\right)^{\eta}\right\}
$$

Take any $0 \leq x<y \leq z \leq 1$.

If $x=0$, then $($ recall $(1.20)) W(0, y, z)=\frac{1}{\sqrt{y z}} \Phi(\sqrt{2 y}, \sqrt{2 z}) \geq b_{0} \frac{z^{\eta}}{\sqrt{y z}}$.

Suppose $x>0$. By (5.10) we see that if $s \in[\sqrt{y}-\sqrt{x}, \sqrt{x}+\sqrt{y}]$, then using definition (1.21) of $Y_{*}$ and the property (6.6) in Appendix we have for all $\theta \in[0,2 \pi]$

$$
Y_{*} \geq\left|\sqrt{z-\frac{\left(x-y+s^{2}\right)^{2}}{4 s^{2}}}-\sqrt{x-\frac{\left(x-y+s^{2}\right)^{2}}{4 s^{2}}}\right| \geq \frac{z-x}{\sqrt{z}+\sqrt{x}}=\sqrt{z}-\sqrt{x}
$$

from which we see that if $s \in[\sqrt{y}, \sqrt{x}+\sqrt{y}]$ then $s+Y_{*} \geq \sqrt{z}$ and so using (5.17) gives

$$
\Phi\left(\sqrt{2} s, \sqrt{2} Y_{*}\right) \geq b_{0} \min \left\{1,\left(s+Y_{*}\right)^{2 \eta}\right\} \geq b_{0} z^{\eta} \quad \forall \theta \in[0,2 \pi]
$$

and thus

$$
W(x, y, z)=\frac{1}{4 \pi \sqrt{x y z}} \int_{\sqrt{y}-\sqrt{x}}^{\sqrt{x}+\sqrt{y}} \mathrm{~d} s \int_{0}^{2 \pi} \Phi\left(\sqrt{2} s, \sqrt{2} Y_{*}\right) \mathrm{d} \theta \geq \frac{b_{0}}{2} \frac{z^{\eta}}{\sqrt{y z}} .
$$

This proves (5.16).

According to (1.17),(1.18) we have a decomposition $\mathcal{J}\left[\varphi_{\varepsilon}\right]=\mathcal{J}^{+}\left[\varphi_{\varepsilon}\right]-\mathcal{J}^{-}\left[\varphi_{\varepsilon}\right]$ where

$$
\begin{aligned}
& \mathcal{J}^{+}\left[\varphi_{\varepsilon}\right](y, z)=\frac{1}{2} \int_{0}^{y+z} W(x, y, z)\left(\varphi_{\varepsilon}(x)+\varphi_{\varepsilon}(y+z-x)\right) \sqrt{x} \mathrm{~d} x, \\
& \mathcal{J}^{-}\left[\varphi_{\varepsilon}\right](y, z)=\frac{1}{2} \int_{0}^{y+z} W(x, y, z) \sqrt{x} \mathrm{~d} x(\varphi(y)+\varphi(z)) .
\end{aligned}
$$

Using the symmetry $\mathcal{J}^{+}\left[\varphi_{\varepsilon}\right](y, z)=\mathcal{J}^{+}\left[\varphi_{\varepsilon}\right](z, y)$ and (5.16) and then omitting $\varphi_{\varepsilon}(y+z-x)$ we compute

$$
\begin{aligned}
& \int_{\mathbb{R}_{\geq 0}^{2}} \mathcal{J}^{+}\left[\varphi_{\varepsilon}\right](y, z) \mathrm{d} F(y) \mathrm{d} F(z) \geq \int_{\frac{1}{2} \varepsilon \leq y \leq z \leq 1} \chi_{y, z} \mathcal{J}^{+}\left[\varphi_{\varepsilon}\right](y, z) \mathrm{d} F(y) \mathrm{d} F(z) \\
& \geq \frac{b_{0}}{4} \int_{\frac{1}{2} \varepsilon \leq y \leq z \leq 1} \chi_{y, z} \frac{z^{\eta}}{\sqrt{y z}}\left(\int_{0}^{\frac{1}{2} \varepsilon} \varphi_{\varepsilon}(x) \sqrt{x} \mathrm{~d} x\right) \mathrm{d} F(y) \mathrm{d} F(z) \\
& \geq \frac{b_{0}}{4} \varepsilon^{3 / 2} \int_{\frac{1}{2} \varepsilon \leq y \leq z \leq 1} \chi_{y, z} \frac{y^{\frac{\eta}{2}} z^{\frac{\eta}{2}}}{\sqrt{y z}}\left(\int_{0}^{\frac{1}{2}}(1-u)^{2} \sqrt{u} \mathrm{~d} u\right) \mathrm{d} F(y) \mathrm{d} F(z) \\
& \geq \frac{b_{0}}{34} \varepsilon^{3 / 2}\left(\int_{\left[\frac{1}{2} \varepsilon, 1\right]} y^{-\frac{1-\eta}{2}} \mathrm{~d} F(y)\right)^{2} .
\end{aligned}
$$


Next from $\Phi(r, \rho) \leq 1$ we have

$$
\begin{aligned}
\mathcal{J}^{-}\left[\varphi_{\varepsilon}\right](y, z) & \leq \frac{\varphi_{\varepsilon}(y)+\varphi_{\varepsilon}(z)}{2}\left(\frac{1}{3} \cdot \frac{y \wedge z}{\sqrt{y \vee z}} \mathbf{1}_{\{y \vee z>0\}}+\sqrt{y \vee z}\right) \\
& \leq \frac{\varphi_{\varepsilon}(y)+\varphi_{\varepsilon}(z)}{2}(\sqrt{y}+\sqrt{z}) \leq \varphi_{\varepsilon}(y) \sqrt{z}+\varphi_{\varepsilon}(z) \sqrt{y}
\end{aligned}
$$

where we used the inequality $\varphi_{\varepsilon}(y) \sqrt{y}+\varphi_{\varepsilon}(z) \sqrt{z} \leq \varphi_{\varepsilon}(y) \sqrt{z}+\varphi_{\varepsilon}(z) \sqrt{y}$ which is because $\left(\varphi_{\varepsilon}(y)-\varphi_{\varepsilon}(z)\right)(\sqrt{y}-\sqrt{z}) \leq 0$ since the function $x \mapsto \varphi_{\varepsilon}(x)$ is non-increasing. Thus we obtain

$$
\begin{aligned}
\int_{\mathbb{R}_{\geq 0}^{2}} \mathcal{J}^{-}\left[\varphi_{\varepsilon}\right](y, z) \mathrm{d} F(y) \mathrm{d} F(z) & \leq \int_{\mathbb{R}_{\geq 0}^{2}}\left(\varphi_{\varepsilon}(y) \sqrt{z}+\varphi_{\varepsilon}(z) \sqrt{y}\right) \mathrm{d} F(y) \mathrm{d} F(z) \\
& =2 M_{1 / 2}(F) N_{0,2}(F, \varepsilon) .
\end{aligned}
$$

This together with the above estimate proves the first inequality (5.13).

Now we prove the second inequality (5.15). It is easily seen that the function $x \mapsto \varphi_{\varepsilon}(x)$ is convex, belongs to $C_{b}^{1,1}\left(\mathbb{R}_{\geq 0}\right)$, and holds the inequality

$$
\Delta_{\operatorname{sym}} \varphi_{\varepsilon}(x, y, z) \geq \frac{(y-x)^{2}}{\varepsilon^{2}} \text { for all } 0 \leq x \leq y \leq z \leq \varepsilon .
$$

Let $0<\varepsilon \leq 1$. By Proposition 5.1 and (5.16), (5.18) we have

$$
\begin{aligned}
\int_{\mathbb{R}_{\geq 0}^{3}} \mathcal{K}\left[\varphi_{\varepsilon}\right] \mathrm{d}^{3} F & \geq \int_{0 \leq x \leq y \leq z \leq \varepsilon, y>0} \chi_{y, z} \frac{b_{0}}{2} \frac{z^{\eta}}{\sqrt{y z}} \frac{(y-x)^{2}}{\varepsilon^{2}} \mathrm{~d} F(x) \mathrm{d} F(y) \mathrm{d} F(z) \\
& =\frac{b_{0}}{2 \varepsilon^{2}} \int_{0<y \leq z \leq \varepsilon} \chi_{y, z} \frac{y^{3 / 2} z^{\eta}}{\sqrt{z}}\left(\int_{[0, y]}(1-x / y)^{2} \mathrm{~d} F(x)\right) \mathrm{d} F(y) \mathrm{d} F(z) \\
& =\frac{b_{0}}{2 \varepsilon^{2}} \int_{0<y \leq z \leq \varepsilon} \chi_{y, z} \frac{y^{\frac{3}{2}+\alpha} z^{\eta}}{\sqrt{z}} N_{\alpha, 2}(F, y) \mathrm{d} F(y) \mathrm{d} F(z) \\
& \geq \frac{b_{0}}{2} \underline{N}_{\alpha, 2}(F, \varepsilon) \frac{1}{\varepsilon^{4+\alpha-\eta}} \int_{0<y \leq z \leq \varepsilon} \chi_{y, z} y^{\frac{3}{2}+\alpha} z^{\frac{3}{2}+\alpha} \mathrm{d} F(y) \mathrm{d} F(z) \\
& =\frac{b_{0}}{2} \underline{N}_{\alpha, 2}(F, \varepsilon) \frac{1}{\varepsilon^{1-\alpha-\eta}}\left(A_{0, p}(F, \varepsilon)\right)^{2}=\frac{b_{0}}{2} \underline{N}_{\alpha, 2}(F, \varepsilon)\left(A_{\beta, p}(F, \varepsilon)\right)^{2} .
\end{aligned}
$$

Finally, assuming $0<\varepsilon \leq 2 / 3,0<\gamma \leq(24)^{-2 / 3}$, we compute

$$
\begin{aligned}
& \int_{\mathbb{R}_{\geq 0}^{3}} \mathcal{K}\left[\varphi_{\varepsilon}\right] \mathrm{d}^{3} F \geq \int_{\mathbb{R}_{\geq 0}^{3}} \mathcal{K}_{1}\left[\varphi_{\varepsilon}\right] \mathrm{d}^{3} F \\
& \geq \int_{0 \leq x<y \leq z \leq \varepsilon} \chi_{y, z} \frac{b_{0}}{2} \frac{z^{\eta}}{\sqrt{y z}} \frac{(y-x)^{2}}{\varepsilon^{2}} \mathrm{~d}^{3} F+\int_{0 \leq x \leq \frac{\varepsilon}{4}, \varepsilon<y \leq z \leq \frac{3}{2} \varepsilon} \chi_{y, z} \frac{b_{0}}{2} \frac{z^{\eta}}{\sqrt{y z}}\left(1-\frac{z+x-y}{\varepsilon}\right)_{+}^{2} \mathrm{~d}^{3} F \\
& \geq \frac{b_{0}}{2 \varepsilon^{2}} \int_{\gamma \varepsilon \leq y \leq z \leq \varepsilon} \chi_{y, z} \frac{y^{3 / 2} N_{0,2}(F, y)}{z^{\frac{1}{2}-\eta}} \mathrm{d}^{2} F+\frac{b_{0}}{2} \frac{1}{16} F\left(\left[0, \frac{\varepsilon}{4}\right]\right) \int_{\varepsilon<y \leq z \leq \frac{3}{2} \varepsilon} \chi_{y, z} \frac{1}{\sqrt{y} z^{\frac{1}{2}-\eta}} \mathrm{d}^{2} F \\
& \geq \frac{b_{0}}{2 \varepsilon^{2}} N_{0,2}(F, \gamma \varepsilon) \frac{(\gamma \varepsilon)^{3 / 2}}{\varepsilon^{\frac{1}{2}-\eta}} \int_{\gamma \varepsilon \leq y \leq z \leq \varepsilon} \chi_{y, z} \mathrm{~d}^{2} F+\frac{b_{0}}{2} \frac{1}{16} N_{0,2}(F, \gamma \varepsilon)\left(\frac{2}{3 \varepsilon}\right)^{1-\eta} \int_{\varepsilon<y \leq z \leq \frac{3}{2} \varepsilon} \chi_{y, z} \mathrm{~d}^{2} F \\
& \geq \frac{b_{0}}{2} N_{0,2}(F, \gamma \varepsilon) \gamma^{3 / 2} \frac{1}{\varepsilon^{1-\eta}}\left((F([\gamma \varepsilon, \varepsilon]))^{2}+\left(F\left(\left(\varepsilon, \frac{3}{2} \varepsilon\right]\right)\right)^{2}\right) \\
& \geq \frac{b_{0}}{4} \frac{\gamma^{3 / 2}}{\varepsilon^{1-\eta}} N_{0,2}(F, \gamma \varepsilon)\left(F\left(\left[\gamma \varepsilon, \frac{3}{2} \varepsilon\right]\right)\right)^{2} .
\end{aligned}
$$


Here we have used $\frac{1}{16}\left(\frac{2}{3}\right)^{1-\eta}>\frac{1}{24} \geq \gamma^{3 / 2}$. This completes the proof.

In the following we will use a convention: in the set $\mathbb{R}_{\geq 0}$ we define the arithmetic operation

$$
\frac{b}{a}=\infty(=+\infty) \quad \text { if } \quad a=0<b
$$

Lemma 5.4. Let $B\left(\mathbf{v}-\mathbf{v}_{*}, \omega\right)$ satisfy Assumption 1.1 with $0 \leq \eta \leq 1 / 2$. Let $F_{t} \in \mathcal{B}_{1}^{+}\left(\mathbb{R}_{\geq 0}\right)$ be a conservative measure-valued isotropic solution of Eq.(1.1) on $[0, \infty)$ with the initial datum $F_{0}$ satisfying $N=N\left(F_{0}\right)>0, E=E\left(F_{0}\right)>0$. Let $c=\sqrt{N E}, 0<\varepsilon \leq 1$. Then

(I) For any $\tau \geq 0, T>0$

$$
N_{0,2}\left(F_{\tau+t}, \varepsilon\right) \geq \frac{b_{0}}{34} \varepsilon^{3 / 2} \inf _{s \in[0, T]}\left(\int_{\left[\frac{1}{2} \varepsilon, 1\right]} y^{-\frac{1-\eta}{2}} \mathrm{~d} F_{\tau+s}(y)\right)^{2} e^{-c t} t \quad \forall t \in[0, T] .
$$

Moreover if $0<\varepsilon \leq 2 / 3,0<\gamma \leq(24)^{-2 / 3}$, then

$$
N_{0,2}\left(F_{\tau+t}, \varepsilon\right) \geq \frac{b_{0}}{4} \frac{\gamma^{3 / 2}}{\varepsilon^{1-\eta}} \inf _{s \in[0, T]}\left(N_{0,2}\left(F_{\tau+s}, \gamma \varepsilon\right)\left(F_{\tau+s}\left(\left[\gamma \varepsilon, \frac{3}{2} \varepsilon\right]\right)\right)^{2}\right) e^{-c t} t \quad \forall t \in[0, T] .
$$

(II) Let $0 \leq \alpha<1-\eta, p=\frac{3}{2}+\alpha, \beta=\frac{1-\alpha-\eta}{2}$, and $h>0$. Then

$$
e^{2 c h} F_{t+h}(\{0\}) \geq N_{0, p}\left(F_{t}, \varepsilon\right)-\left(\frac{2 e^{3 c h} N}{h b_{0} \underline{N}_{\alpha, 2}\left(F_{t}, \varepsilon\right)}\right)^{1 / 2}\left(\frac{p}{\beta}\right)^{p} \varepsilon^{\beta} \quad \forall t \geq 0 .
$$

In particular for $\alpha=0$ we have

$$
e^{2 c h} F_{t+h}(\{0\}) \geq N_{0,3 / 2}\left(F_{t}, \varepsilon\right)-\left(\frac{2 e^{3 c h} N}{h b_{0} F_{t}(\{0\})}\right)^{1 / 2}\left(\frac{3}{1-\eta}\right)^{3 / 2} \varepsilon^{\frac{1-\eta}{2}} \quad \forall t \geq 0 .
$$

Proof. (I): Since $\varphi_{\varepsilon}(x)=\left[(1-x / \varepsilon)_{+}\right]^{2}$ is convex and belongs to $C_{b}^{1,1}\left(\mathbb{R}_{\geq 0}\right)$, we deduce from Proposition 5.1, Lemma 5.3, and $M_{1 / 2}\left(F_{\tau+t}\right) \leq \sqrt{N E}=c$ that

$$
\begin{aligned}
& \frac{\mathrm{d}}{\mathrm{d} t} N_{0,2}\left(F_{t}, \varepsilon\right)=\int_{\mathbb{R}_{\geq 0}^{2}} \mathcal{J}\left[\varphi_{\varepsilon}\right] \mathrm{d}^{2} F_{t}+\int_{\mathbb{R}_{\geq 0}^{3}} \mathcal{K}\left[\varphi_{\varepsilon}\right] \mathrm{d}^{3} F_{t} \\
& \geq \frac{b_{0}}{34} \varepsilon^{3 / 2}\left(\int_{\left[\frac{1}{2} \varepsilon, 1\right]} y^{-\frac{1-\eta}{2}} \mathrm{~d} F_{t}(y)\right)^{2}-2 M_{1 / 2}\left(F_{t}\right) N_{0,2}\left(F_{t}, \varepsilon\right) \\
& \geq \frac{b_{0}}{34} \varepsilon^{3 / 2} \inf _{s \in[\tau, \tau+T]}\left(\int_{\left[\frac{1}{2} \varepsilon, 1\right]} y^{-\frac{1-\eta}{2}} \mathrm{~d} F_{s}(y)\right)^{2}-2 c N_{0,2}\left(F_{t}, \varepsilon\right), \quad t \in[\tau, \tau+T]
\end{aligned}
$$

and so

$$
\begin{aligned}
& N_{0,2}\left(F_{\tau+t}, \varepsilon\right) \geq N_{0,2}\left(F_{\tau}, \varepsilon\right) e^{-2 c t}+\frac{b_{0}}{34} \varepsilon^{3 / 2} \inf _{s \in[\tau, \tau+T]}\left(\int_{\left[\frac{1}{2} \varepsilon, 1\right]} y^{-\frac{1-\eta}{2}} \mathrm{~d} F_{s}(y)\right)^{2} \frac{1-e^{-2 c t}}{2 c} \\
& \geq \frac{b_{0}}{34} \varepsilon^{3 / 2} \inf _{s \in[0, T]}\left(\int_{\left[\frac{1}{2} \varepsilon, 1\right]} y^{-\frac{1-\eta}{2}} \mathrm{~d} F_{\tau+s}(y)\right)^{2} e^{-c t} t, \quad t \in[0, T]
\end{aligned}
$$

where we used $1-e^{-x} \geq x e^{-x / 2}, x \geq 0$. This proves the first inequality. 
Next suppose that $0<\varepsilon \leq 2 / 3,0<\gamma \leq(24)^{-2 / 3}$. Then using Lemma 5.2 and Lemmas 5.3 we have

$$
\frac{\mathrm{d}}{\mathrm{d} t} N_{0,2}\left(F_{t}, \varepsilon\right) \geq-2 c N_{0,2}\left(F_{t}, \varepsilon\right)+\frac{b_{0}}{4} \frac{\gamma^{3 / 2}}{\varepsilon^{1-\eta}} \inf _{s \in[\tau, \tau+T]}\left(N_{0,2}\left(F_{s}, \gamma \varepsilon\right)\left(F_{s}\left(\left[\gamma \varepsilon, \frac{3}{2} \varepsilon\right]\right)\right)^{2}\right)
$$

for all $t \in[\tau, \tau+T]$. As shown above we also obtain

$$
N_{0,2}\left(F_{\tau+t}, \varepsilon\right) \geq \frac{b_{0}}{4} \frac{\gamma^{3 / 2}}{\varepsilon^{1-\eta}} \inf _{s \in[0, T]}\left(N_{0,2}\left(F_{\tau+s}, \gamma \varepsilon\right)\left(F_{\tau+s}\left(\left[\gamma \varepsilon, \frac{3}{2} \varepsilon\right]\right)\right)^{2}\right) e^{-c t} t, \quad t \in[0, T] .
$$

(II): By Lemma 5.2, (5.14) and that $t \mapsto c^{c t} \underline{N}_{\alpha, 2}\left(F_{t}, \varepsilon\right)$ is non-decreasing, we have

$$
e^{c t} N_{0,2}\left(F_{t}, \varepsilon\right)-e^{c s} N_{0,2}\left(F_{s}, \varepsilon\right) \geq \frac{b_{0}}{2} e^{c s} \underline{N}_{\alpha, 2}(s, \varepsilon) \int_{s}^{t}\left(A_{\beta, p}\left(F_{\tau}, \varepsilon\right)\right)^{2} \mathrm{~d} \tau, \quad 0 \leq s<t .
$$

Now for any $h>0$, letting $s$ and $t$ be replaced with $t$ and $t+h$ respectively and noting that $N_{0,2}\left(F_{t+h}, \varepsilon\right) \leq N$, we obtain

$$
e^{c h} N \geq \frac{b_{0}}{2} \underline{N}_{\alpha, 2}\left(F_{t}, \varepsilon\right) \int_{t}^{t+h}\left(A_{\beta, p}\left(F_{\tau}, \varepsilon\right)\right)^{2} \mathrm{~d} \tau .
$$

Then, using Cauchy-Schwarz inequality,

$$
\frac{1}{h} \int_{t}^{t+h} A_{\beta, p}\left(F_{\tau}, \varepsilon\right) \mathrm{d} \tau \leq\left(\frac{1}{h} \int_{t}^{t+h}\left(A_{\beta, p}\left(F_{\tau}, \varepsilon\right)\right)^{2} \mathrm{~d} \tau\right)^{1 / 2} \leq\left(\frac{2 e^{c h} N}{h b_{0} \underline{N}_{\alpha, 2}\left(F_{t}, \varepsilon\right)}\right)^{1 / 2} .
$$

Note that according to the convention (5.19), this inequality still holds when $\underline{N}_{\alpha, 2}\left(F_{t}, \varepsilon\right)=0$.

On the other hand, applying Lemma 2.3 in [21] to the measure $F=F_{\tau}$ we have

$$
N_{0, p}\left(F_{\tau}, \varepsilon\right) \leq F_{\tau}(\{0\})+\left(\frac{p}{\beta} \varepsilon^{\frac{\beta}{p}}\right)^{p-1} \int_{0}^{\varepsilon} \varepsilon_{1}^{-1+\frac{\beta}{p}} A_{\beta, p}\left(F_{\tau}, \varepsilon_{1}\right) \mathrm{d} \varepsilon_{1}, \quad \varepsilon>0 .
$$

Taking integration and using the fact that $0<\varepsilon_{1} \leq \varepsilon \Longrightarrow \underline{N}_{\alpha, 2}\left(F_{t}, \varepsilon_{1}\right) \geq \underline{N}_{\alpha, 2}\left(F_{t}, \varepsilon\right)$ we have

$$
\begin{aligned}
& \frac{1}{h} \int_{t}^{t+h} N_{0, p}\left(F_{\tau}, \varepsilon\right) \mathrm{d} \tau \leq \frac{1}{h} \int_{t}^{t+h} F_{\tau}(\{0\}) \mathrm{d} \tau+\left(\frac{p}{\beta} \varepsilon^{\frac{\beta}{p}}\right)^{p-1} \int_{0}^{\varepsilon} \varepsilon_{1}^{-1+\frac{\beta}{p}} \frac{1}{h} \int_{t}^{t+h} A_{\beta, p}\left(F_{\tau}, \varepsilon_{1}\right) \mathrm{d} \tau \mathrm{d} \varepsilon_{1} \\
& \leq \frac{1}{h} \int_{t}^{t+h} F_{\tau}(\{0\}) \mathrm{d} \tau+\left(\frac{p}{\beta} \varepsilon^{\frac{\beta}{p}}\right)^{p-1} \int_{0}^{\varepsilon} \varepsilon_{1}^{-1+\frac{\beta}{p}}\left(\frac{2 e^{c h} N}{h b_{0} \underline{N}_{\alpha, 2}\left(F_{t}, \varepsilon_{1}\right)}\right)^{1 / 2} \mathrm{~d} \varepsilon_{1} \\
& \leq \frac{1}{h} \int_{t}^{t+h} F_{\tau}(\{0\}) \mathrm{d} \tau+\left(\frac{2 e^{c h} N}{h b_{0} \underline{N}_{\alpha, 2}\left(F_{t}, \varepsilon\right)}\right)^{1 / 2}\left(\frac{p}{\beta}\right)^{p} \varepsilon^{\beta} .
\end{aligned}
$$

Since $t \mapsto e^{c t} N_{0, p}\left(F_{t}, \varepsilon\right), t \mapsto e^{c t} F_{t}(\{0\})$ are non-decreasing on $[0, \infty)$, it follows that $e^{-c h} N_{0, p}\left(F_{t}, \varepsilon\right) \leq$ $N_{0, p}\left(F_{\tau}, \varepsilon\right), F_{\tau}(\{0\}) \leq e^{c h} F_{t+h}(\{0\})$ for all $\tau \in[t, t+h]$ and so

$$
e^{-c h} N_{0, p}\left(F_{t}, \varepsilon\right) \leq e^{c h} F_{t+h}(\{0\})+\left(\frac{2 e^{c h} N}{h b_{0} \underline{N}_{\alpha, 2}\left(F_{t}, \varepsilon\right)}\right)^{1 / 2}\left(\frac{p}{\beta}\right)^{p} \varepsilon^{\beta} .
$$

This gives (5.22) 
Finally for the case $\alpha=0$, i.e. $\beta=(1-\eta) / 2, p=3 / 2$, we have $\underline{N}_{0,2}\left(F_{t}, \varepsilon\right)=F_{t}(\{0\})$ and thus (5.23) holds true.

The following lemma and proposition are key steps for obtaining lower bounds of $F_{t}(\{0\})$ and for the convergence of $F_{t}(\{0\})$ to BEC without additional condition on the initial data.

Lemma 5.5. Let $B\left(\mathbf{v}-\mathbf{v}_{*}, \omega\right)$ satisfy Assumption 1.1 with $0 \leq \eta<1 / 4$. Given any $N>$ $0, E>0$. Let $\alpha=\frac{1}{10}(1-4 \eta)$,

$$
A_{\alpha}^{*}=\left(\frac{4 \cdot b_{0}^{-2 / 7}}{1-\left(\frac{2}{3}\right)^{\alpha / 4}}\right)^{7 / 3}, \quad 0<\varepsilon \leq \min \left\{\left(\frac{\alpha \log \left(\frac{3}{2}\right)}{8 \sqrt{N E}}\right)^{1 / \alpha}, \frac{2}{3},\left(\frac{3}{2}\right)^{1 / 4}\left(\frac{N}{A_{\alpha}^{*}}\right)^{1 / \alpha}\right\}
$$

and let $F_{t} \in \mathcal{B}_{1}^{+}\left(\mathbb{R}_{\geq 0}\right)$ be a conservative measure-valued isotropic solution of Eq.(1.1) on $[0, \infty)$ with initial datum $F_{0}$ satisfying $N\left(F_{0}\right)=N, E\left(F_{0}\right)=E$. Suppose for some $\tau \geq 0$

$$
N_{0,2}\left(F_{\tau}, \frac{3}{2} \varepsilon\right) \geq\left(\frac{2}{3}\right)^{\alpha / 4} A_{\alpha}^{*} \varepsilon^{\alpha} .
$$

Then

$$
N_{0,2}\left(F_{\tau+2 \varepsilon^{\alpha}}, \varepsilon\right) \geq e^{-2 c \varepsilon^{\alpha}}\left(\frac{2}{3}\right)^{\alpha} N_{0,2}\left(F_{\tau}, \frac{3}{2} \varepsilon\right)
$$

where $c=\sqrt{N E}$.

Proof. Since $t \mapsto e^{c t} N_{0,2}\left(F_{t}, \varepsilon\right)$ is non-decreasing on $[0, \infty)$, it suffices to prove that

$$
\exists t \in\left[0,2 \varepsilon^{\alpha}\right] \quad \text { s.t. } \quad N_{0,2}\left(F_{\tau+t}, \varepsilon\right) \geq\left(\frac{2}{3}\right)^{\alpha} N_{0,2}\left(F_{\tau}, \frac{3}{2} \varepsilon\right) .
$$

We use contradiction argument. Suppose that (5.27) does not hold. Then we have

$$
N_{0,2}\left(F_{\tau+t}, \varepsilon\right)<\left(\frac{2}{3}\right)^{\alpha} N_{0,2}\left(F_{\tau}, \frac{3}{2} \varepsilon\right) \quad \forall t \in\left[0,2 \varepsilon^{\alpha}\right] .
$$

For convenience we denote

$$
M=N_{0,2}\left(F_{\tau}, \frac{3}{2} \varepsilon\right), \quad \gamma=1-\left(\frac{2}{3}\right)^{\alpha / 4} .
$$

We have $\gamma<1-\left(\frac{2}{3}\right)^{1 / 40}<(24)^{-2 / 3}$ (because $\alpha \leq 1 / 10$ ), and

$$
N_{0,2}\left(F_{\tau+t}, \varepsilon\right) \geq \int_{[0, \gamma \varepsilon]}\left(1-\frac{x}{\varepsilon}\right)^{2} \mathrm{~d} F_{\tau+t}(x) \geq(1-\gamma)^{2} F_{\tau+t}([0, \gamma \varepsilon]) .
$$

Noting that

$$
0 \leq t \leq 2 \varepsilon^{\alpha} \leq \frac{\alpha \log \left(\frac{3}{2}\right)}{4 c} \quad \Longrightarrow \quad e^{-c t} \geq e^{-2 \varepsilon^{\alpha}} \geq\left(\frac{2}{3}\right)^{\alpha / 4}=1-\gamma
$$


and using (5.29) and (5.28) we have

$$
\begin{aligned}
& F_{\tau+t}\left(\left[\gamma \varepsilon, \frac{3}{2} \varepsilon\right]\right)=F_{\tau+t}\left(\left[0, \frac{3}{2} \varepsilon\right]\right)-F_{\tau+t}([0, \gamma \varepsilon)) \\
& \geq N_{0,2}\left(F_{\tau+t}, \frac{3}{2} \varepsilon\right)-\frac{1}{(1-\gamma)^{2}} N_{0,2}\left(F_{\tau+t}, \varepsilon\right) \geq e^{-c t} N_{0,2}\left(F_{\tau}, \frac{3}{2} \varepsilon\right)-\frac{1}{(1-\gamma)^{2}}\left(\frac{2}{3}\right)^{\alpha} M \\
& \geq\left(\frac{2}{3}\right)^{\alpha / 4} M-\frac{1}{(1-\gamma)^{2}}\left(\frac{2}{3}\right)^{\alpha} M=(1-\gamma) \gamma M \quad \forall t \in\left[0,2 \varepsilon^{\alpha}\right] .
\end{aligned}
$$

Combining this with the assumption $\frac{3}{2} \varepsilon \leq 1$ gives

$$
\left(\int_{\left[\frac{1}{2} \gamma \varepsilon, 1\right]} y^{-\frac{1-\eta}{2}} \mathrm{~d} F_{\tau+t}(y)\right)^{2} \geq\left(\frac{3}{2} \varepsilon\right)^{-(1-\eta)}\left(F_{\tau+t}\left(\left[\frac{1}{2} \gamma \varepsilon, \frac{3}{2} \varepsilon\right]\right)\right)^{2} \geq\left(\frac{2}{3 \varepsilon}\right)^{1-\eta}[(1-\gamma) \gamma M]^{2}
$$

for all $t \in\left[0,2 \varepsilon^{\alpha}\right]$. Inserting this into (5.20) in Lemma 5.4 we obtain

$$
N_{0,2}\left(F_{\tau+t}, \gamma \varepsilon\right) \geq \frac{b_{0}}{34}\left(\frac{2}{3}\right)^{1-\eta}(1-\gamma)^{2} \gamma^{\frac{3}{2}+2} M^{2} \varepsilon^{\frac{1}{2}+\eta} t e^{-c t} \quad \forall t \in\left[0,2 \varepsilon^{\alpha}\right] .
$$

Using (5.31),(5.32) and (5.30) to $t=\varepsilon^{\alpha}+s, s \in\left[0, \varepsilon^{\alpha}\right]$, we have

$$
\begin{aligned}
& N_{0,2}\left(F_{\tau+\varepsilon^{\alpha}+s}, \gamma \varepsilon\right)\left(F_{\tau+\varepsilon^{\alpha}+s}\left(\left[\gamma \varepsilon, \frac{3}{2} \varepsilon\right]\right)\right)^{2} \\
& \geq \frac{b_{0}}{34}\left(\frac{2}{3}\right)^{1-\eta}(1-\gamma)^{2} \gamma^{\frac{3}{2}+2} M^{2} \varepsilon^{\frac{1}{2}+\eta}\left(\varepsilon^{\alpha}+s\right) e^{-c\left(\varepsilon^{\alpha}+s\right)}((1-\gamma) \gamma M)^{2} \\
& \geq \frac{b_{0}}{34}\left(\frac{2}{3}\right)^{1-\eta}(1-\gamma)^{5} \gamma^{\frac{3}{2}+4} M^{4} \varepsilon^{\frac{1}{2}+\eta+\alpha} \quad \forall s \in\left[0, \varepsilon^{\alpha}\right] .
\end{aligned}
$$

Then using (5.21) in Lemma 5.4 where $\tau$ is replaced by $\tau+\varepsilon^{\alpha}$ and taking $t=T=\varepsilon^{\alpha}$, we compute

$$
\begin{aligned}
& N_{0,2}\left(F_{\tau+2 \varepsilon^{\alpha}}, \varepsilon\right) \geq \frac{b_{0}}{4} \frac{\gamma^{3 / 2}}{\varepsilon^{1-\eta}} e^{-c \varepsilon^{\alpha}} \varepsilon^{\alpha} \inf _{s \in\left[0, \varepsilon^{\alpha}\right]}\left(N_{0,2}\left(F_{\tau+\varepsilon^{\alpha}+s}, \gamma \varepsilon\right)\left(F_{\tau+\varepsilon^{\alpha}+s}\left(\left[\frac{1}{2} \gamma \varepsilon, \frac{3}{2} \varepsilon\right]\right)\right)^{2}\right) \\
& \geq \frac{b_{0}}{4} \frac{\gamma^{3 / 2}}{\varepsilon^{1-\eta}} e^{-c \varepsilon^{\alpha}} \varepsilon^{\alpha} \frac{b_{0}}{34}\left(\frac{2}{3}\right)^{1-\eta}(1-\gamma)^{5} \gamma^{3 / 2+4} M^{4} \varepsilon^{\frac{1}{2}+\eta+\alpha} \\
& \left.\geq \frac{b_{0}^{2}}{136}\left(\frac{2}{3}\right)^{1-\eta}(1-\gamma)^{6} \gamma^{7}\left(\frac{M}{\varepsilon^{\alpha}}\right)^{3} M \quad \text { (because } e^{-c \varepsilon^{\alpha}} \geq 1-\gamma \text { and } \frac{1}{2}-2 \eta-2 \alpha=3 \alpha\right) \\
& \left.\geq \frac{b_{0}^{2}}{136}\left(\frac{2}{3}\right)^{1-\eta}(1-\gamma)^{9} \gamma^{7}\left(A_{\alpha}^{*}\right)^{3} M \quad \text { (because, by }(5.25), M \geq(1-\gamma) A_{\alpha}^{*} \varepsilon^{\alpha}\right) \\
& =\frac{4^{7}}{136}\left(\frac{2}{3}\right)^{1-\eta+\frac{9}{4} \alpha} M>50\left(\frac{2}{3}\right)^{\alpha} M=50\left(\frac{2}{3}\right)^{\alpha} N_{0,2}\left(F_{\tau}, \frac{3}{2} \varepsilon\right)
\end{aligned}
$$

which contradicts (5.28). Thus (5.26) holds true.

Proposition 5.6. Let $B\left(\mathbf{v}-\mathbf{v}_{*}, \omega\right)$ satisfy Assumption 1.1 with $0 \leq \eta<1 / 4$, and let $\alpha=$ $\frac{1}{10}(1-4 \eta)$

$$
A_{\alpha}^{*}=\left(\frac{4 \cdot b_{0}^{-2 / 7}}{1-\left(\frac{2}{3}\right)^{\alpha / 4}}\right)^{7 / 3}, \quad B_{\alpha}^{*}=\left(1-\left(\frac{2}{3}\right)^{\alpha}\right) \frac{\alpha \log \left(\frac{3}{2}\right)}{8}, \quad C_{\alpha}^{*}=\frac{\sqrt{b_{0}}}{87}\left(A_{\alpha}^{*}\right)^{3 / 2} .
$$


Given any $N>0, E>0$, let $F_{t} \in \mathcal{B}_{1}^{+}\left(\mathbb{R}_{\geq 0}\right)$ be a conservative measure-valued isotropic solution of Eq.(1.1) on $[0, \infty)$ with initial datum $F_{0}$ satisfying $N\left(F_{0}\right)=N, E\left(F_{0}\right)=E$, and suppose for some $\tau \geq 0$ and

$$
0<\varepsilon \leq \min \left\{\left(\frac{B_{\alpha}^{*}}{\sqrt{N E}}\right)^{1 / \alpha}, \frac{2}{3},\left(\frac{C_{\alpha}^{*}}{N^{3 / 4} E^{1 / 4}}\right)^{\frac{2}{1-3 \alpha-\eta}},\left(\frac{N}{A_{\alpha}^{*}}\right)^{1 / \alpha}\right\}
$$

$F_{\tau}$ satisfies

$$
N_{0,2}\left(F_{\tau}, \frac{3}{2} \varepsilon\right) \geq A_{\alpha}^{*} \varepsilon^{\alpha}
$$

Then, at the time $t_{\varepsilon}=\tau+2\left(1-\left(\frac{2}{3}\right)^{\alpha}\right)^{-1} \varepsilon^{\alpha}+\frac{1}{3 \sqrt{N E}}$, it holds

$$
F_{t_{\varepsilon}}(\{0\})>\frac{1}{5} A_{\alpha}^{*} \varepsilon^{\alpha}
$$

hence

$$
F_{t}(\{0\}) \geq e^{-c\left(t-t_{\varepsilon}\right)} F_{t_{\varepsilon}}(\{0\})>0 \quad \forall t \geq t_{\varepsilon}
$$

where $c=\sqrt{N E}$.

Proof. Step1. Let $h_{\varepsilon}^{*}=2\left(1-\left(\frac{2}{3}\right)^{\alpha}\right)^{-1} \varepsilon^{\alpha}$. We prove that

$$
\underline{N}_{\alpha, 2}\left(F_{\tau+h_{\varepsilon}^{*}}, \varepsilon\right) \geq \frac{0.9}{\varepsilon^{\alpha}} N_{0,2}\left(F_{\tau}, \frac{3}{2} \varepsilon\right) .
$$

We will use Lemma 5.5 with an iteration argument. Let

$$
\varepsilon_{k}=\left(\frac{2}{3}\right)^{k} \varepsilon, \quad h_{n}=2 \sum_{k=0}^{n}\left(\varepsilon_{k}\right)^{\alpha}=2 \sum_{k=0}^{n}\left(\frac{2}{3}\right)^{k \alpha} \varepsilon^{\alpha}, \quad k, n=0,1,2, \ldots .
$$

and let $M=N_{0,2}\left(F_{\tau}, \frac{3}{2} \varepsilon\right)$. We first prove that for all $n=0,1,2, \ldots$,

$$
N_{0,2}\left(F_{\tau+h_{n}}, \varepsilon_{n}\right) \geq e^{-c h_{n}}\left(\frac{2}{3}\right)^{\alpha(n+1)} M
$$

From (5.26) in Lemma 5.5 we see that (5.39) holds for $n=0$. Suppose that (5.39) holds for some $n \in \mathbb{N} \cup\{0\}$. From (5.33), (5.34) we have

$$
h_{n} \leq h_{\varepsilon}^{*}=2\left(1-\left(\frac{2}{3}\right)^{\alpha}\right)^{-1} \varepsilon^{\alpha} \leq \frac{\alpha \log \left(\frac{3}{2}\right)}{4 c} \text { hence } e^{-c h_{n}} \geq e^{-c h_{\varepsilon}^{*}} \geq\left(\frac{2}{3}\right)^{\alpha / 4} .
$$

Then

$$
\frac{e^{-c h_{n}}\left(\frac{2}{3}\right)^{\alpha(n+1)} M}{\left(\varepsilon_{n+1}\right)^{\alpha}}=e^{-c h_{n}} \frac{M}{\varepsilon^{\alpha}} \geq\left(\frac{2}{3}\right)^{\alpha / 4} A_{\alpha}^{*}
$$

which together with the inductive hypotheses implies that (using $\frac{3}{2} \varepsilon_{n+1}=\varepsilon_{n}$ )

$$
\begin{aligned}
& N_{0,2}\left(F_{\tau+h_{n}}, \frac{3}{2} \varepsilon_{n+1}\right)=N_{0,2}\left(F_{\tau+h_{n}}, \varepsilon_{n}\right) \geq e^{-c h_{n}}\left(\frac{2}{3}\right)^{\alpha(n+1)} M \geq\left(\frac{2}{3}\right)^{\alpha / 4} A_{\alpha}^{*}\left(\varepsilon_{n+1}\right)^{\alpha} \\
& \text { and } \varepsilon_{n+1}=\left(\frac{2}{3}\right)^{n+1} \varepsilon<\varepsilon, \quad\left(\varepsilon_{n+1}\right)^{\alpha}<\frac{1}{2} h_{\varepsilon}^{*} \leq \frac{\alpha \log \left(\frac{3}{2}\right)}{8 c} .
\end{aligned}
$$


Then using Lemma 5.5 and noting that $h_{n+1}=h_{n}+2\left(\varepsilon_{n+1}\right)^{\alpha}$ we have

$$
\begin{aligned}
& N_{0,2}\left(F_{\tau+h_{n+1}}, \varepsilon_{n+1}\right)=N_{0,2}\left(F_{\left.\tau+h_{n}+2\left(\varepsilon_{n+1}\right)^{\alpha}, \varepsilon_{n+1}\right)}\right. \\
& \geq e^{-2 c\left(\varepsilon_{n+1}\right)^{\alpha}}\left(\frac{2}{3}\right)^{\alpha} N_{0,2}\left(F_{\tau+h_{n}}, \frac{3}{2} \varepsilon_{n+1}\right)=e^{-2 c\left(\varepsilon_{n+1}\right)^{\alpha}}\left(\frac{2}{3}\right)^{\alpha} N_{0,2}\left(F_{\tau+h_{n}}, \varepsilon_{n}\right) \\
& \geq e^{-2 c\left(\varepsilon_{n+1}\right)^{\alpha}}\left(\frac{2}{3}\right)^{\alpha} e^{-c h_{n}}\left(\frac{2}{3}\right)^{\alpha(n+1)} M=e^{-c h_{n+1}}\left(\frac{2}{3}\right)^{\alpha(n+2)} M .
\end{aligned}
$$

Therefore (5.39) holds also for $n+1$ and thus, by induction, (5.39) holds for all $n \in \mathbb{N} \cup\{0\}$.

Using the non-decrease of $t \mapsto e^{c t} N_{0,2}\left(F_{t}, \varepsilon\right)$ and (5.39) we have

$$
\begin{aligned}
& N_{0,2}\left(F_{\tau+h_{\varepsilon}^{*}},\left(\frac{2}{3}\right)^{n} \varepsilon\right) \geq e^{-c\left(h_{\varepsilon}^{*}-h_{n}\right)} N_{0,2}\left(F_{\tau+h_{n}},\left(\frac{2}{3}\right)^{n} \varepsilon\right) \\
& \geq e^{-c\left(h_{\varepsilon}^{*}-h_{n}\right)} e^{-c h_{n}}\left(\frac{2}{3}\right)^{\alpha(n+1)} M=e^{-c h_{\varepsilon}^{*}}\left(\frac{2}{3}\right)^{\alpha(n+1)} M, \quad n=0,1,2, \ldots .
\end{aligned}
$$

Now for any $0<\delta \leq \varepsilon$, there is $n \in \mathbb{N}$ such that $\left(\frac{2}{3}\right)^{n} \varepsilon<\delta \leq\left(\frac{2}{3}\right)^{n-1} \varepsilon$, so we have

$$
\begin{aligned}
& \frac{1}{\delta^{\alpha}} N_{0,2}\left(F_{\tau+h_{\varepsilon}^{*}}, \delta\right) \geq \frac{1}{\delta^{\alpha}} N_{0,2}\left(F_{\tau+h_{\varepsilon}^{*}},\left(\frac{2}{3}\right)^{n} \varepsilon\right) \\
& \geq \frac{1}{\left(\left(\frac{2}{3}\right)^{n-1} \varepsilon\right)^{\alpha}} e^{-c h_{\varepsilon}^{*}}\left(\frac{2}{3}\right)^{\alpha(n+1)} M \geq\left(\frac{2}{3}\right)^{\left(2+\frac{1}{4}\right) \alpha} \frac{M}{\varepsilon^{\alpha}}>0.9 \frac{M}{\varepsilon^{\alpha}}
\end{aligned}
$$

where the last inequality is due to $0<\alpha \leq \frac{1}{10}$. Thus

$$
\underline{N}_{\alpha, 2}\left(F_{\tau+h_{\varepsilon}^{*}}, \varepsilon\right)=\inf _{0<\delta \leq \varepsilon} \frac{N_{0,2}\left(F_{\tau+h_{\varepsilon}^{*}}, \delta\right)}{\delta^{\alpha}} \geq 0.9 \frac{M}{\varepsilon^{\alpha}}
$$

i.e. (5.38) holds true.

Step2. Let $\tau_{\varepsilon}=\tau+h_{\varepsilon}^{*}$. Using Lemma 5.4 (recall there $p=\frac{3}{2}+\alpha, \beta=\frac{1-\alpha-\eta}{2}$ ) with $h=\frac{1}{3 c}=\frac{1}{3 \sqrt{N E}}, t=\tau_{\varepsilon}$, and using $p<2$ and the inequality (5.38) to deduce

$$
\frac{1}{\varepsilon^{\alpha}} N_{0, p}\left(F_{\tau_{\varepsilon}}, \varepsilon\right) \geq \frac{1}{\varepsilon^{\alpha}} N_{0,2}\left(F_{\tau_{\varepsilon}}, \varepsilon\right) \geq \underline{N}_{\alpha, 2}\left(F_{\tau_{\varepsilon}}, \varepsilon\right) \geq \frac{0.9}{\varepsilon^{\alpha}} N_{0,2}\left(F_{\tau}, \frac{3}{2} \varepsilon\right) \geq 0.9 A_{\alpha}^{*}
$$

we obtain

$$
\begin{aligned}
& e^{2 / 3} F_{\tau_{\varepsilon}+h}(\{0\}) \geq N_{0, p}\left(F_{\tau_{\varepsilon}}, \varepsilon\right)-\left(\frac{2 e^{3 c h} N}{h b_{0} \underline{N}_{\alpha, 2}\left(F_{\tau_{\varepsilon}}, \varepsilon\right)}\right)^{1 / 2}\left(\frac{p}{\beta}\right)^{p} \varepsilon^{\beta} \\
& \geq 0.9 A_{\alpha}^{*} \varepsilon^{\alpha}-\left(\frac{6 e N^{3 / 2} E^{1 / 2}}{b_{0} 0.9 A_{\alpha}^{*}}\right)^{1 / 2}\left(\frac{3+2 \alpha}{1-\alpha-\eta}\right)^{\frac{3}{2}+\alpha} \varepsilon^{\beta} \geq \frac{0.9}{2} A_{\alpha}^{*} \varepsilon^{\alpha}
\end{aligned}
$$

where for the last inequality we used $\beta-\alpha=\frac{1-3 \alpha-\eta}{2}>0, \varepsilon^{\beta-\alpha} \leq \frac{C_{\alpha}^{*}}{N^{3 / 4} E^{1 / 4}}$, and

$$
\left(\frac{6 e}{0.9}\right)^{1 / 2}\left(\frac{3+2 \alpha}{1-\alpha-\eta}\right)^{\frac{3}{2}+\alpha} \frac{1}{87} \leq\left(\frac{6 e}{0.9}\right)^{1 / 2} \frac{4^{8 / 5}}{87}<\frac{0.9}{2}
$$

Thus, at the time $t_{\varepsilon}:=\tau_{\varepsilon}+h=\tau+2\left(1-\left(\frac{2}{3}\right)^{\alpha}\right)^{-1} \varepsilon^{\alpha}+\frac{1}{3 \sqrt{N E}}$, we obtain

$$
F_{t_{\varepsilon}}(\{0\})=F_{\tau_{\varepsilon}+h}(\{0\}) \geq \frac{0.9}{2 e^{2 / 3}} A_{\alpha}^{*} \varepsilon^{\alpha}>\frac{1}{5} A_{\alpha}^{*} \varepsilon^{\alpha} .
$$

The inequality (5.37) follows from this and the non-decrease of $t \mapsto F_{t}(\{0\}) e^{c t}$ on $[0, \infty)$. This completes the proof. 
Remark 5.7. In comparison with the previous results in [14], [21] on the occurrence of condensation (i.e. $F_{t}(\{0\})>0$ ) in finite time, Proposition 5.6 not only provides a simple condition (5.35) with $\tau=0$ for the initial data $F_{0}$, but also gives an explicit and useful lower bound (5.36) for condensation. Note that the last term $\left(\frac{N}{A_{\alpha}^{*}}\right)^{1 / \alpha}$ in $\min \{\cdots\}$ in (5.34) (same thing for $\left(\frac{3}{2}\right)^{1 / 4}\left(\frac{N}{A_{\alpha}^{*}}\right)^{1 / \alpha}$ in $\left.(5.24)\right)$ is just for $\varepsilon$ to be in a possible range. In fact, from $N \geq N_{0,2}\left(F_{\tau}, \varepsilon\right)$ one sees that the inequality (5.35) implies $N \geq A_{\alpha}^{*} \varepsilon^{\alpha}$. In applications of Proposition 5.6 the number $\varepsilon$ will be chosen much less than $\left(\frac{N}{A_{\alpha}^{*}}\right)^{1 / \alpha}$. See below.

Example of bounded initial data. Here we show that for any $N>0, E>0$, there are many bounded and smooth initial data that satisfy the condition in Proposition 5.6 for $\tau=0$. Let $\alpha, A_{\alpha}^{*}, B_{\alpha}^{*}, C_{\alpha}^{*}$ be given in Proposition 5.6. For any $N>0, E>0$, let

$$
\begin{aligned}
& 0<\varepsilon \leq \min \left\{\left(\frac{B_{\alpha}^{*}}{\sqrt{N E}}\right)^{1 / \alpha}, \frac{2}{3},\left(\frac{C_{\alpha}^{*}}{N^{3 / 4} E^{1 / 4}}\right)^{\frac{2}{1-3 \alpha-\eta}}, \frac{E}{2 N},\left(\frac{N}{27 A_{\alpha}^{*}}\right)^{1 / \alpha}\right\}, \\
& \delta=3 \frac{E}{N}, \quad a=\frac{2 E}{\varepsilon(\delta-\varepsilon)}, \quad b=\frac{2 N\left(\frac{2 E}{N}-\varepsilon\right)}{\delta(\delta-\varepsilon)}, \\
& g_{0}(x)=a \mathbf{1}_{\left[\frac{1}{4} \varepsilon, \frac{3}{4} \varepsilon\right]}(x)+b \mathbf{1}_{\left[\frac{1}{4} \delta, \frac{3}{4} \delta\right]}(x), \quad \mathrm{d} G_{0}(x)=g_{0}(x) \mathrm{d} x .
\end{aligned}
$$

We compute (notice that $\varepsilon \leq \frac{1}{6} \delta$ )

$$
\begin{aligned}
& N\left(G_{0}\right)=\frac{1}{2}(a \varepsilon+b \delta)=N, \quad E\left(G_{0}\right)=\frac{1}{4}\left(a \varepsilon^{2}+b \delta^{2}\right)=E \\
& G_{0}([0, \varepsilon])=\frac{1}{2} a \varepsilon=\frac{E}{3 \frac{E}{N}-\varepsilon}>\frac{N}{3} \geq 9 A_{\alpha}^{*} \varepsilon^{\alpha}
\end{aligned}
$$

Let $J(x)=c_{1} e^{-\frac{1}{1-x^{2}}} \mathbf{1}_{(-1,1)}(x)$ where $c_{1}>0$ is such that $\int_{\mathbb{R}} J(x) \mathrm{d} x=1$. Let $J_{\lambda}(x)=\frac{1}{\lambda} J\left(\frac{x}{\lambda}\right)(\lambda>$ 0 ), choose $\lambda=\frac{1}{8} \varepsilon$, and consider $f_{0}(x)=\frac{1}{\sqrt{x}}\left(J_{\lambda} * g_{0}\right)(x)$ (convolution). It is easily seen that $0 \leq f_{0} \in C_{c}^{\infty}(\mathbb{R})$ and $\operatorname{supp} f_{0} \subset\left[\frac{1}{4} \varepsilon-\lambda, \frac{3}{4} \delta+\lambda\right]=\left[\frac{1}{8} \varepsilon, \frac{3}{4} \delta+\frac{1}{8} \varepsilon\right]$. Let $F_{0} \in \mathcal{B}_{1}^{+}\left(\mathbb{R}_{\geq 0}\right)$ be defined by $\mathrm{d} F_{0}(x)=f_{0}(x) \sqrt{x} \mathrm{~d} x$. By simple calculation (using $\delta \geq 6 \varepsilon$ ) we have

$$
N\left(F_{0}\right)=N\left(G_{0}\right)=N, \quad E\left(F_{0}\right)=E\left(G_{0}\right)=E, \quad F_{0}([0, \varepsilon])=G_{0}([0, \varepsilon]) \geq 9 A_{\alpha}^{*} \varepsilon^{\alpha} .
$$

Thus $\left(\right.$ since $\left(1-\frac{x}{\frac{3}{2} \varepsilon}\right)^{2} \geq \frac{1}{9}$ for all $x \in[0, \varepsilon]$ )

$$
N_{0,2}\left(F_{0}, \frac{3}{2} \varepsilon\right) \geq \frac{1}{9} F_{0}([0, \varepsilon]) \geq A_{\alpha}^{*} \varepsilon^{\alpha}
$$

So $F_{0}$ (with the number $\varepsilon$ ) satisfies the condition (5.35) in Proposition 5.6 for $\tau=0$.

Theorem 5.8. Let $B\left(\mathbf{v}-\mathbf{v}_{*}, \omega\right)$ satisfy Assumption 1.1 with $0 \leq \eta<1 / 4$, and let $F_{0} \in$ $\mathcal{B}_{1}^{+}\left(\mathbb{R}_{\geq 0}\right)$ with $N:=N\left(F_{0}\right)>0, E:=E\left(F_{0}\right)>0$ satisfy the low temperature condition $\bar{T} / \bar{T}_{c}=$ $2.2720 \frac{E}{N^{5 / 3}}<1$. Let $F_{t} \in \mathcal{B}_{1}^{+}\left(\mathbb{R}_{\geq 0}\right)$ with the initial datum $F_{0}$ be a conservative measure-valued 
isotropic solution of Eq.(1.1) on $[0, \infty)$ obtained in Proposition 3.4. Then, for any $0<\lambda<\frac{1}{19}$ we have

$$
\left|F_{t}(\{0\})-\left(1-\left(\bar{T} / \bar{T}_{c}\right)^{3 / 5}\right) N\right| \leq C(1+t)^{-\frac{1-\eta}{2(4-\eta)} \lambda} \quad \forall t \geq 0
$$

where the constant $C>0$ depends only on $N, E, b_{0}, \eta$ and $\lambda$.

Proof. Let $N_{0}=F_{\text {be }}(\{0\})=\left(1-\left(\bar{T} / \bar{T}_{c}\right)^{3 / 5}\right) N$. From Lemma 2.1, Theorem4.4 we have

$$
R(t):=\sup _{\tau \geq t}\left\|F_{\tau}-F_{\text {be }}\right\|_{1}^{\circ} \leq C_{1} \sup _{\tau \geq t} \sqrt{S\left(F_{\text {be }}\right)-S\left(F_{\tau}\right)} \leq C_{2}(1+t)^{-\lambda / 2} \quad \forall t \geq 0
$$

where here and below $C>0, C_{i}>0(i=1,2, \ldots, 8), \tau_{0}>0$ and $t_{0}>1$ are finite constants that depend only on $N, E, b_{0}, \eta$ and $\lambda$.

Step1. Let $A_{\alpha}^{*}, B_{\alpha}^{*}, C_{\alpha}^{*}$ be given in Proposition 5.6 with $\alpha=\frac{1}{10}(1-4 \eta)$, let

$$
\varepsilon_{0}=\min \left\{\left(\frac{B_{\alpha}^{*}}{\sqrt{N E}}\right)^{1 / \alpha}, \frac{2}{3},\left(\frac{C_{\alpha}^{*}}{N^{3 / 4} E^{1 / 4}}\right)^{\frac{2}{1-3 \alpha-\eta}},\left(\frac{N_{0}}{2 A_{\alpha}^{*}}\right)^{1 / \alpha}\right\}
$$

and let $k_{0}=2\left(1-\left(\frac{2}{3}\right)^{\alpha}\right)^{-1} \varepsilon_{0}{ }^{\alpha}+\frac{1}{3 \sqrt{N E}}$. We prove that there is $\tau_{0}>0$ such that

$$
F_{t}(\{0\}) \geq \frac{1}{5} A_{\alpha}^{*} \varepsilon_{0}^{\alpha} \quad \forall t \geq \tau_{0}+k_{0}
$$

Let $F_{\text {be }}$ be the unique Bose-Einstein distribution having the mass and energy $N, E$. For any $\varepsilon>0, p \geq 1$, using the conservation of mass and $0 \leq 1-\left[(1-x / \varepsilon)_{+}\right]^{p} \leq \frac{p}{\varepsilon} x$ we have

$$
\left|N_{0, p}\left(F_{t}, \varepsilon\right)-N_{0, p}\left(F_{\text {be }}, \varepsilon\right)\right|=\left|\int_{\mathbb{R}_{\geq 0}}\left(1-\left[(1-x / \varepsilon)_{+}\right]^{p}\right) \mathrm{d}\left(F_{t}-F_{\text {be }}\right)(x)\right| \leq \frac{p}{\varepsilon}\left\|F_{t}-F_{\text {be }}\right\|_{1}^{\circ} .
$$

From this and $N_{0, p}\left(F_{\text {be }}, \varepsilon\right) \geq F_{\text {be }}(\{0\})=N_{0}$ we obtain

$$
N_{0, p}\left(F_{t}, \varepsilon\right) \geq N_{0, p}\left(F_{\text {be }}, \varepsilon\right)-\frac{p}{\varepsilon}\left\|F_{t}-F_{\text {be }}\right\|_{1}^{\circ} \geq N_{0}-\frac{p}{\varepsilon}\left\|F_{t}-F_{\text {be }}\right\|_{1}^{\circ} .
$$

Since $\left\|F_{t}-F_{\text {be }}\right\|_{1}^{\circ} \leq C_{2}(1+t)^{-\lambda / 2}$, there is $\tau_{0}>0$ such that

$$
\frac{2}{\varepsilon_{0}}\left\|F_{t}-F_{\text {be }}\right\|_{1}^{\circ}<\frac{N_{0}}{2} \quad \forall t \geq \tau_{0} \quad \text { hence } \quad N_{0, p}\left(F_{t}, \varepsilon_{0}\right) \geq \frac{1}{2} N_{0} \quad \forall t \geq \tau_{0}, \forall 1 \leq p \leq 2 .
$$

Note that $\varepsilon \mapsto N_{0,2}\left(F_{t}, \varepsilon\right)$ is non-decreasing. We then deduce from the choice of $\varepsilon_{0}$ that

$$
N_{0,2}\left(F_{t}, \frac{3}{2} \varepsilon_{0}\right) \geq N_{0,2}\left(F_{t}, \varepsilon_{0}\right) \geq \frac{1}{2} N_{0} \geq A_{\alpha}^{*} \varepsilon_{0}^{\alpha} \quad \forall t \geq \tau_{0}
$$

Thus by Proposition 5.6 we conclude $F_{t+k_{0}}(\{0\}) \geq \frac{1}{5} A_{\alpha}^{*} \varepsilon_{0}^{\alpha}$. Since this holds for all $t \geq \tau_{0}$, we obtain (5.41).

Step2. According to (5.40), we need only prove that there is $t_{0}>1$ such that

$$
\left|F_{t}(\{0\})-N_{0}\right| \leq C(R(t-1))^{\frac{1-\eta}{4-\eta}} \quad \forall t \geq t_{0} .
$$


Using (5.23) (see Lemma 5.4), (5.43) for $p=3 / 2$, the lower bound (5.41), and denoting $c=$ $\sqrt{N E}, C_{3}=\frac{1}{5} A_{\alpha}^{*} \varepsilon_{0}^{\alpha}$, we have for all $0<h \leq 1,0<\varepsilon \leq 1$

$$
\begin{aligned}
F_{t}(\{0\}) & \geq e^{-2 c h} N_{0,3 / 2}\left(F_{t-h}, \varepsilon\right)-e^{-2 c h}\left(\frac{2 e^{3 c h} N}{h b_{0} F_{t-h}(\{0\})}\right)^{1 / 2}\left(\frac{3}{1-\eta}\right)^{3 / 2} \varepsilon^{\frac{1-\eta}{2}} \\
& \geq(1-2 c h) N_{0}-\frac{3}{2 \varepsilon}\left\|F_{t-h}-F_{\mathrm{be}}\right\|_{1}^{\circ}-\left(\frac{2 N}{h b_{0} C_{3}}\right)^{1 / 2}\left(\frac{3}{1-\eta}\right)^{3 / 2} \varepsilon^{\frac{1-\eta}{2}} \\
& \geq(1-2 c h) N_{0}-\frac{3}{2 \varepsilon} R(t-h)-C_{4} h^{-\frac{1}{2}} \varepsilon^{\frac{1-\eta}{2}} \quad \forall t \geq \tau_{0}+k_{0}+h .
\end{aligned}
$$

Here we used $e^{-2 c h} \geq 1-2 c h, e^{-2 c h} e^{3 c h / 2}<1$. Now we consider

$$
0<\varepsilon \leq C_{5}:=\min \left\{1,\left(\frac{2 c N_{0}}{C_{4}}\right)^{\frac{2}{1-\eta}}\right\}, \quad h=h_{\varepsilon}=\left(\frac{C_{4}}{2 c N_{0}}\right)^{2 / 3} \varepsilon^{\frac{1-\eta}{3}} .
$$

For all $0<\varepsilon \leq C_{5}$ we have $0<h_{\varepsilon} \leq 1$ and from (5.45) we obtain

$$
F_{t}(\{0\}) \geq N_{0}-\frac{3}{2 \varepsilon} R(t-1)-C_{6} \varepsilon^{\frac{1-\eta}{3}} \quad \forall t \geq \tau_{0}+k_{0}+1 .
$$

Since $R(t-1) \leq C_{2} t^{-\lambda / 2}$, we choose $t_{0} \geq \tau_{0}+k_{0}+1$ large enough such that $(R(t-1))^{\frac{3}{4-\eta}}<C_{5}$ for all $t \geq t_{0}$. Then for every $t \geq t_{0}$, taking $\varepsilon=(R(t-1))^{\frac{3}{4-\eta}}$ we obtain from (5.46) that

$$
F_{t}(\{0\}) \geq N_{0}-C_{7}(R(t-1))^{\frac{1-\eta}{4-\eta}} \quad \forall t \geq t_{0} .
$$

On the other hand using the inequality (5.42) for $p=1$ we have

$$
\begin{aligned}
& F_{t}(\{0\}) \leq N_{0,1}\left(F_{t}, \varepsilon\right) \leq N_{0,1}\left(F_{\mathrm{be}}, \varepsilon\right)+\frac{1}{\varepsilon}\left\|F_{t}-F_{\mathrm{be}}\right\|_{1}^{\circ} \\
& =F_{\mathrm{be}}(\{0\})+\int_{(0, \varepsilon]}\left(1-\frac{x}{\varepsilon}\right) \frac{1}{e^{x / \kappa}-1} \sqrt{x} \mathrm{~d} x+\frac{1}{\varepsilon}\left\|F_{t}-F_{\mathrm{be}}\right\|_{1}^{\circ} \\
& \leq N_{0}+2 \kappa \sqrt{\varepsilon}+\frac{1}{\varepsilon} R(t) \quad \forall t \geq 0, \quad \forall \varepsilon>0 .
\end{aligned}
$$

Minimizing the right hand side of $(5.48)$ with respect to $\varepsilon \in(0, \infty)$ gives

$$
F_{t}(\{0\}) \leq N_{0}+C_{8}(R(t))^{1 / 3} \leq N_{0}+C_{8}(R(t-1))^{\frac{1-\eta}{4-\eta}} \quad \forall t \geq t_{0} .
$$

Combining (5.47) and (5.49) we obtain (5.44) with the constant $C=\max \left\{C_{7}, C_{8}\right\}$.

Finally at the end of this section we finish the proof of Theorem 1.4:

Proof of Theorem 1.4. We need only prove the algebraic decay rate of $\left\|F-F_{\mathrm{be}}\right\|_{1}$ since the algebraic decay rate of $S\left(F_{\mathrm{be}}\right)-S\left(F_{t}\right)$ has been proven by Theorem 4.4. Let $C_{i}(i=1,2, \ldots, 8)$ denote finite positive constants that depend only on $N, E, b_{0}, \eta$ and $\lambda$. Since $F_{t}$ conserves the mass and energy, it follows from Lemma 2.1 and Theorem 4.4 that

$$
\left\|F-F_{\text {be }}\right\|_{1}^{\circ} \leq C_{1}\left(S\left(F_{\text {be }}\right)-S\left(F_{t}\right)\right)^{1 / 2} \leq C_{2}(1+t)^{-\lambda / 2} \quad \forall t \geq 0 .
$$


Now if $\bar{T} / \bar{T}_{c}<1$, then by Lemma 2.1, Theorem 5.8, and (5.50) we have

$$
\begin{aligned}
& \left\|F_{t}-F_{\text {be }}\right\|_{1} \leq 2\left|F_{t}(\{0\})-F_{\text {be }}(\{0\})\right|+C_{3}\left(\left\|F_{t}-F_{\text {be }}\right\|_{1}^{\circ}\right)^{1 / 3} \\
& \leq C_{4}(1+t)^{-\frac{1-\eta}{2(4-\eta)} \lambda}+C_{5}(1+t)^{-\lambda / 6} \leq C_{6}(1+t)^{-\frac{1-\eta}{2(4-\eta)} \lambda} \quad \forall t \geq 0
\end{aligned}
$$

while if $\bar{T} / \bar{T}_{c} \geq 1$, then we have from Lemma 2.1 and (5.50) that

$$
\left\|F_{t}-F_{\text {be }}\right\|_{1} \leq C_{7}\left(\left\|F-F_{\text {be }}\right\|_{1}^{\circ}\right)^{1 / 3} \leq C_{8}(1+t)^{-\lambda / 6} \leq C_{8}(1+t)^{-\frac{1-\eta}{2(4-\eta)} \lambda} \quad \forall t \geq 0 .
$$

This completes the proof of Theorem 1.4.

\section{Appendix}

Here we prove some properties that have been used in the previous sections.

6.1. Some integral equalities. We will use the following integral formula:

(1) (Carleman Representation). Let $\Psi$ be a Borel measurable function on $\mathbb{R}^{3} \times \mathbb{R}^{3}$ and it is nonnegative or satisfies some integrability such that the following integrals make sense)

$$
\int_{\mathbb{R}^{3} \times \mathbb{S}^{2}} \Psi\left(\mathbf{v}^{\prime}, \mathbf{v}_{*}^{\prime}\right) \mathrm{d} \omega \mathrm{d} \mathbf{v}_{*}=2 \int_{\mathbb{R}^{3}} \frac{\mathrm{d} \mathbf{x}}{|\mathbf{x}|^{2}} \int_{\mathbb{R}^{2}(\mathbf{x})} \Psi(\mathbf{v}-\mathbf{x}, \mathbf{v}-\mathbf{y}) \mathrm{d} \mathbf{y}
$$

for almost all $\mathbf{v} \in \mathbb{R}^{3}$, where $\left(\mathbf{v}^{\prime}, \mathbf{v}_{*}^{\prime}\right)$ is given in the $\omega$-representation $(1.2)$ and $\mathrm{d} \mathbf{y}$ in $\int_{\mathbb{R}^{2}(\mathbf{x})}\{\cdots\} \mathrm{d} \mathbf{y}$ is the Lebesgue measure element on the plan $\mathbb{R}^{2}(\mathbf{x})=\left\{\mathbf{y} \in \mathbb{R}^{3} \mid \mathbf{y} \perp \mathbf{x}\right\}$.

(2) (see e.g.[18]). Let $\Psi$ be continuous on $\left(\mathbb{R}^{3} \backslash\{0\}\right) \times\left(\mathbb{R}^{3} \backslash\{0\}\right)$ and suppose that $\Psi$ is nonnegative or generally such that the following integrals makes sense. Then we have

$$
\int_{\mathbb{R}^{3}} \frac{\mathrm{d} \mathbf{x}}{|\mathbf{x}|} \int_{\mathbb{R}^{2}(\mathbf{x})} \Psi(\mathbf{x}, \mathbf{y}) \mathrm{d} \mathbf{y}=\int_{\mathbb{R}^{3}} \frac{\mathrm{d} \mathbf{y}}{|\mathbf{y}|} \int_{\mathbb{R}^{2}(\mathbf{y})} \Psi(\mathbf{x}, \mathbf{y}) \mathrm{d} \mathbf{x} .
$$

(3) Let $\Psi$ be continuous on $\mathbb{R}_{>0}^{2}$ and suppose that $\Psi$ is nonnegative or generally such that the following integrals make sense. Then for any $\mathbf{x} \in \mathbb{R}^{3} \backslash\{0\}$ we have

$$
\int_{\mathbb{R}^{2}(\mathbf{x})} \Psi(|\mathbf{y}|,|\mathbf{v}-\mathbf{y}|) \mathrm{d} \mathbf{y}=\int_{\left|\mathbf{v}_{\mathbf{x}}\right|}^{\infty} r_{*}^{\prime} \mathrm{d} r_{*}^{\prime} \int_{0}^{2 \pi} \Psi\left(\left|\sqrt{{r_{*}^{\prime}}^{2}-\left|\mathbf{v}_{\mathbf{x}}\right|^{2}}+e^{\mathrm{i} \theta} \sqrt{|\mathbf{v}|^{2}-\left|\mathbf{v}_{\mathbf{x}}\right|^{2}}\right|, r_{*}^{\prime}\right) \mathrm{d} \theta
$$

where $\mathbf{v}_{\mathbf{x}}=\left(\mathbf{v} \cdot \frac{\mathbf{x}}{|\mathbf{x}|}\right) \frac{\mathbf{x}}{|\mathbf{x}|}, \mathrm{i}=\sqrt{-1}$. Here for real numbers $a, b, \theta$ we just use $\left|a+e^{\mathrm{i} \theta} b\right|=\left(a^{2}+b^{2}+\right.$ $2 a b \cos (\theta))^{1 / 2}$ to shorten notation.

By identity $\left|\mathbf{v}_{*}\right|^{2}=\left|\mathbf{v}^{\prime}\right|^{2}+\left|\mathbf{v}_{*}^{\prime}\right|^{2}-|\mathbf{v}|^{2}$, any function of $\left(\ldots,|\mathbf{v}|^{2} / 2,\left|\mathbf{v}_{*}\right|^{2} / 2,\left|\mathbf{v}^{\prime}\right|^{2} / 2,\left|\mathbf{v}_{*}^{\prime}\right|^{2} / 2\right)$ will be automatically written as a function of $\left(\ldots,|\mathbf{v}|^{2} / 2,\left|\mathbf{v}^{\prime}\right|^{2} / 2,\left|\mathbf{v}_{*}^{\prime}\right|^{2} / 2\right)$.

In the following, for any $(x, y, z, s, \theta) \in \mathbb{R}_{\geq 0}^{4} \times[0,2 \pi]$, we denote as the above that $x_{*}=$ $(y+z-x)_{+}$and let $Y_{*}=Y_{*}(x, y, z, s, \theta)$ be given by $(1.21)$ and $\widetilde{Y}_{*}$ be defined by $Y_{*}(\cdot)$ with exchanging $y \leftrightarrow z$ i.e.

$$
\widetilde{Y}_{*}=Y_{*}(x, z, y, s, \theta) .
$$


Lemma 6.1. Let $\Psi \in C\left(\mathbb{R}_{\geq 0}^{5}\right.$ ) be such that the following integrals make sense ( for instance $\Psi \geq 0$ on $\mathbb{R}_{\geq 0}^{5}$ or $\Psi$ is such that integral is absolutely convergent). Then for any $\mathbf{v} \in \mathbb{R}^{3} \backslash\{0\}$ we have with $x=|\mathbf{v}|^{2} / 2=r^{2} / 2$ that

$$
\begin{aligned}
& \int_{\mathbb{R}^{3} \times \mathbb{S}^{2}} \frac{\left|\left(\mathbf{v}-\mathbf{v}_{*}\right) \cdot \omega\right|}{(4 \pi)^{2}} \Psi\left(\left|\mathbf{v}-\mathbf{v}^{\prime}\right|,\left|\mathbf{v}-\mathbf{v}_{*}^{\prime}\right|,|\mathbf{v}|^{2} / 2,\left|\mathbf{v}^{\prime}\right|^{2} / 2,\left|\mathbf{v}_{*}^{\prime}\right|^{2} / 2\right) \mathrm{d} \omega \mathrm{d} \mathbf{v}_{*} \\
& =\frac{1}{4 \pi \sqrt{x}} \int_{\mathbb{R}_{\geq 0}^{2}} 1_{\{y+z>x\}} \mathrm{d} y \mathrm{~d} z \int_{|\sqrt{x}-\sqrt{y}| \vee\left|\sqrt{x_{*}}-\sqrt{z}\right|}^{(\sqrt{x}+\sqrt{y}) \wedge\left(\sqrt{x_{*}}+\sqrt{z}\right)} \int_{0}^{2 \pi} \Psi\left(\sqrt{2} s, \sqrt{2} Y_{*}, x, y, z\right) \mathrm{d} \theta \mathrm{d} s \\
& =\frac{1}{4 \pi \sqrt{x}} \int_{\mathbb{R}_{\geq 0}^{2}} 1_{\{y+z>x\}} \mathrm{d} y \mathrm{~d} z \int_{|\sqrt{x}-\sqrt{z}| \vee\left|\sqrt{x_{*}}-\sqrt{y}\right|}^{(\sqrt{x}+\sqrt{z}) \wedge\left(\sqrt{x_{*}}+\sqrt{y}\right)} \int_{0}^{2 \pi} \Psi\left(\sqrt{2} \widetilde{Y}_{*}, \sqrt{2} s, x, y, z\right) \mathrm{d} \theta \mathrm{d} s .
\end{aligned}
$$

Proof. Fix any $\mathbf{v}=r \sigma=\sqrt{2 x} \sigma\left(x>0, \sigma \in \mathbb{S}^{2}\right)$. Let $I(\mathbf{v})$ be the left hand side of (6.4). Note that $\left|\left(\mathbf{v}-\mathbf{v}_{*}\right) \cdot \omega\right|=\left|\mathbf{v}-\mathbf{v}^{\prime}\right|$. Then using (6.1),(6.3) and simple changes of variables and denoting $r_{*}=\sqrt{\left(r^{\prime 2}+r_{*}^{\prime 2}-r^{2}\right)_{+}}$we compute

$$
\begin{aligned}
& (4 \pi)^{2} I(\mathbf{v})=2 \int_{\mathbb{R}^{3}} \frac{\mathrm{d} \mathbf{x}}{|\mathbf{x}|} \int_{\mathbb{R}^{2}(\mathbf{x})} \Psi\left(|\mathbf{x}|,|\mathbf{y}|,|\mathbf{v}|^{2} / 2,|\mathbf{v}-\mathbf{x}|^{2} / 2,|\mathbf{v}-\mathbf{y}|^{2} / 2\right) \mathrm{d} \mathbf{y} \\
& =2 \int_{\mathbb{R}^{3}} \frac{\mathrm{d} \mathbf{x}}{|\mathbf{x}|} \int_{\left|\mathbf{v}_{\mathbf{x}}\right|}^{\infty} r_{*}^{\prime} \mathrm{d} r_{*}^{\prime} \int_{0}^{2 \pi} \Psi\left(|\mathbf{x}|,\left|\sqrt{r_{*}^{\prime 2}-\left|\mathbf{v}_{\mathbf{x}}\right|^{2}}+e^{\mathrm{i} \theta} \sqrt{|\mathbf{v}|^{2}-\left|\mathbf{v}_{\mathbf{x}}\right|^{2}}\right|, \frac{r^{2}}{2}, \frac{|\mathbf{v}-\mathbf{x}|^{2}}{2}, \frac{{r_{*}^{\prime}}^{2}}{2}\right) \mathrm{d} \theta \\
& =2 \int_{\mathbb{R}^{3}} \frac{\mathrm{d} \mathbf{x}}{|\mathbf{v}-\mathbf{x}|} \int_{\mid \mathbf{v} \cdot \mathbf{v}-\mathbf{x}}^{|\mathbf{v}-\mathbf{x}|} r_{*}^{\prime} \mathrm{d} r_{*}^{\prime} \int_{0}^{2 \pi} \\
& \times \Psi\left(|\mathbf{v}-\mathbf{x}|,\left|\sqrt{{r_{*}^{\prime}}^{2}-\left|\mathbf{v} \cdot \frac{\mathbf{v}-\mathbf{x}}{|\mathbf{v}-\mathbf{x}|}\right|^{2}}+e^{\mathrm{i} \theta} \sqrt{|\mathbf{v}|^{2}-\left|\mathbf{v} \cdot \frac{\mathbf{v}-\mathbf{x}}{|\mathbf{v}-\mathbf{x}|}\right|^{2}}\right|, \frac{r^{2}}{2}, \frac{|\mathbf{x}|^{2}}{2}, \frac{r_{*}^{\prime 2}}{2}\right) \mathrm{d} \theta \\
& =\frac{4 \pi}{r} \int_{\mathbb{R}_{\geq 0}^{2}, r^{\prime 2}+r_{*}^{\prime 2}>r^{2}} r^{\prime} r_{*}^{\prime} \mathrm{d} r^{\prime} \mathrm{d} r_{*}^{\prime} \int_{\left|r^{\prime}-r\right| \vee\left|r_{*}^{\prime}-r_{*}\right|}^{\left(r^{\prime}+r\right) \wedge\left(r_{*}+r_{*}^{\prime}\right)} \mathrm{d} s \\
& \times \int_{0}^{2 \pi} \Psi\left(s,\left|\sqrt{r_{*}^{\prime 2}-\frac{\left(r^{2}-r^{\prime 2}+s^{2}\right)^{2}}{4 s^{2}}}+e^{\mathrm{i} \theta} \sqrt{r^{2}-\frac{\left(r^{2}-r^{\prime 2}+s^{2}\right)^{2}}{4 s^{2}}}\right|, \frac{r^{2}}{2}, \frac{r^{\prime 2}}{2}, \frac{r_{*}^{\prime 2}}{2}\right) \mathrm{d} \theta \\
& =\frac{4 \pi}{\sqrt{x}} \int_{\mathbb{R}_{\geq 0}^{2}, y+z>x} \mathrm{~d} y \mathrm{~d} z \int_{|\sqrt{x}-\sqrt{y}| \vee\left|\sqrt{x_{*}}-\sqrt{z}\right|}^{(\sqrt{x}+\sqrt{y}) \wedge\left(\sqrt{x_{*}}+\sqrt{z}\right)} \int_{0}^{2 \pi} \Psi\left(\sqrt{2} s, \sqrt{2} Y_{*}, x, y, z\right) \mathrm{d} \theta \mathrm{d} s
\end{aligned}
$$

where in the above calculation we have used the following properties: if $r>0, r^{\prime}>0, r_{*}^{\prime}>$ $0, r_{*}>0,-1<t<1, s>0$, then

$r_{*}^{\prime}>\frac{\left|r^{2}-r r^{\prime} t\right|}{\sqrt{r^{2}+r^{\prime 2}-2 r r^{\prime} t}} \Longrightarrow r^{\prime 2}+r_{*}^{\prime 2}>r^{2} ; \quad r_{*}^{\prime}>\frac{\left|r^{2}-r^{\prime 2}+s^{2}\right|}{2 s} \Longleftrightarrow\left|r_{*}^{\prime}-r_{*}\right|<s<r_{*}^{\prime}+r_{*}$.

By the way, we also have the following relation

$$
\begin{aligned}
& |\sqrt{x}-\sqrt{y}| \vee\left|\sqrt{x_{*}}-\sqrt{z}\right| \leq s \leq(\sqrt{x}+\sqrt{y}) \wedge\left(\sqrt{x_{*}}+\sqrt{z}\right) \text { and } s>0 \\
& \Longrightarrow \quad z-\frac{\left(x-y+s^{2}\right)^{2}}{4 s^{2}} \geq 0 \text { and } x-\frac{\left(x-y+s^{2}\right)^{2}}{4 s^{2}} \geq 0
\end{aligned}
$$

which is useful when dealing with $Y_{*}=Y_{*}(x, y, z, s, \theta)$. 
Next using the formula (6.2) and (6.5) with exchanges $\mathbf{x} \leftrightarrow \mathbf{y}, y \leftrightarrow z$ we also have

$$
\begin{aligned}
(4 \pi)^{2} I(\mathbf{v}) & =2 \int_{\mathbb{R}^{3}} \frac{\mathrm{d} \mathbf{y}}{|\mathbf{y}|} \int_{\mathbb{R}^{2}(\mathbf{x})} \Psi\left(|\mathbf{x}|,|\mathbf{y}|,|\mathbf{v}|^{2} / 2,|\mathbf{v}-\mathbf{x}|^{2} / 2,|\mathbf{v}-\mathbf{y}|^{2} / 2\right) \mathrm{d} \mathbf{x} \\
& =2 \int_{\mathbb{R}^{3}} \frac{\mathrm{d} \mathbf{x}}{|\mathbf{x}|} \int_{\mathbb{R}^{2}(\mathbf{y})} \Psi\left(|\mathbf{y}|,|\mathbf{x}|,|\mathbf{v}|^{2} / 2,|\mathbf{v}-\mathbf{y}|^{2} / 2,|\mathbf{v}-\mathbf{x}|^{2} / 2\right) \mathrm{d} \mathbf{y} \\
& =\frac{4 \pi}{\sqrt{x}} \int_{\mathbb{R}_{\geq 0}^{2}, y+z>x} \mathrm{~d} y \mathrm{~d} z \int_{|\sqrt{x}-\sqrt{y}| \vee\left|\sqrt{x_{*}}-\sqrt{z}\right|}^{(\sqrt{x}+\sqrt{y}) \wedge\left(\sqrt{x_{*}}+\sqrt{z}\right)} \int_{0}^{2 \pi} \Psi\left(\sqrt{2} Y_{*}, \sqrt{2} s, x, z, y\right) \mathrm{d} \theta \mathrm{d} s \\
& =\frac{4 \pi}{\sqrt{x}} \int_{\mathbb{R}_{\geq 0}^{2}, y+z>x} \mathrm{~d} y \mathrm{~d} z \int_{|\sqrt{x}-\sqrt{z}| \vee\left|\sqrt{x_{*}}-\sqrt{y}\right|}^{(\sqrt{x}+\sqrt{z}) \wedge\left(\sqrt{x_{*}}+\sqrt{y}\right)} \int_{0}^{2 \pi} \Psi\left(\sqrt{2} \widetilde{Y}_{*}, \sqrt{2} s, x, y, z\right) \mathrm{d} \theta \mathrm{d} s .
\end{aligned}
$$

Lemma 6.2. Let $\Psi \in C\left(\mathbb{R}_{\geq 0}^{5}\right)$. Then for any $y>0, z>0$

$$
\begin{aligned}
& \int_{\mathbb{S}^{2} \times \mathbb{S}^{2}} \mathrm{~d} \sigma \mathrm{d} \sigma_{*} \int_{\mathbb{S}^{2}} \frac{\left|\left(\mathbf{v}-\mathbf{v}_{*}\right) \cdot \omega\right|}{(4 \pi)^{2}} \Psi\left(\left|\mathbf{v}-\mathbf{v}^{\prime}\right|,\left|\mathbf{v}-\mathbf{v}_{*}^{\prime}\right|,|\mathbf{v}|^{2} / 2,\left|\mathbf{v}^{\prime}\right|^{2} / 2,\left|\mathbf{v}_{*}^{\prime}\right|^{2} / 2\right) \mathrm{d} \omega \\
& =\frac{1}{\sqrt{2 y z}} \int_{0}^{y+z} \mathrm{~d} x \int_{|\sqrt{y}-\sqrt{x}| \mathrm{v}\left|\sqrt{x_{*}}-\sqrt{z}\right|}^{(\sqrt{y}+\sqrt{x}) \wedge\left(\sqrt{z}+\sqrt{x_{*}}\right)} \int_{0}^{2 \pi} \Psi\left(\sqrt{2} s, \sqrt{2} Y_{*}, x, y, z\right) \mathrm{d} \theta \mathrm{d} s
\end{aligned}
$$

where in the left integral $\mathbf{v}=\sqrt{2 y} \sigma, \mathbf{v}_{*}=\sqrt{2 z} \sigma_{*}$.

Proof. It is easily seen that both sides of (6.7) are continuous in $(y, z) \in \mathbb{R}_{>0}^{2}$. Take any $\psi \in C_{c}\left(\mathbb{R}_{\geq 0}^{2}\right)$. We compute with change of variables and using Lemma 6.1

$$
\begin{aligned}
& \int_{\mathbb{R}_{\geq 0}^{2}} \psi(y, z) \sqrt{y} \sqrt{z}\left(\int_{\mathbb{S}^{2} \times \mathbb{S}^{2}} \mathrm{~d} \sigma \mathrm{d} \sigma_{*} \int_{\mathbb{S}^{2}} \frac{\left|\left(\mathbf{v}-\mathbf{v}_{*}\right) \cdot \omega\right|}{(4 \pi)^{2}}\right. \\
& \left.\times\left.\Psi\left(\left|\mathbf{v}-\mathbf{v}^{\prime}\right|,\left|\mathbf{v}-\mathbf{v}_{*}^{\prime}\right|,|\mathbf{v}|^{2} / 2,\left|\mathbf{v}^{\prime}\right|^{2} / 2,\left|\mathbf{v}_{*}^{\prime}\right|^{2} / 2\right)\right|_{\mathbf{v}=\sqrt{2 y} \sigma, \mathbf{v}_{*}=\sqrt{2 z} \sigma_{*}} \mathrm{~d} \omega\right) \mathrm{d} y \mathrm{~d} z \\
& =\frac{1}{2} \int_{\mathbb{R}^{3} \times \mathbb{R}^{3} \times \mathbb{S}^{2}} \psi\left(|\mathbf{v}|^{2} / 2,\left|\mathbf{v}_{*}\right|^{2} / 2\right) \frac{\left|\left(\mathbf{v}-\mathbf{v}_{*}\right) \cdot \omega\right|}{(4 \pi)^{2}} \\
& \times \Psi\left(\left|\mathbf{v}-\mathbf{v}^{\prime}\right|,\left|\mathbf{v}-\mathbf{v}_{*}^{\prime}\right|,|\mathbf{v}|^{2} / 2,\left|\mathbf{v}^{\prime}\right|^{2} / 2,\left|\mathbf{v}_{*}^{\prime}\right|^{2} / 2\right) \mathrm{d} \omega \mathrm{d} \mathbf{v} \mathrm{d} \mathbf{v}_{*} \\
& =\frac{1}{2} \int_{\mathbb{R}^{3} \times \mathbb{R}^{3} \times \mathbb{S}^{2}} \psi\left(\left|\mathbf{v}^{\prime}\right|^{2} / 2,\left|\mathbf{v}_{*}^{\prime}\right|^{2} / 2\right) \frac{\left|\left(\mathbf{v}-\mathbf{v}_{*}\right) \cdot \omega\right|}{(4 \pi)^{2}} \\
& \times \Psi\left(\left|\mathbf{v}-\mathbf{v}^{\prime}\right|,\left|\mathbf{v}-\mathbf{v}_{*}^{\prime}\right|,|\mathbf{v}|^{2} / 2,\left|\mathbf{v}^{\prime}\right|^{2} / 2,\left|\mathbf{v}_{*}^{\prime}\right|^{2} / 2\right) \mathrm{d} \omega \mathrm{d} \mathbf{v} \mathrm{d} \mathbf{v}_{*} \\
& =\int_{\mathbb{R}_{\geq 0}^{2}} \psi(y, z) \sqrt{y z}\left(\frac{1}{\sqrt{2 y z}} \int_{0}^{y+z} \mathrm{~d} x \int_{|\sqrt{x}-\sqrt{y}| \mathbf{v}\left|\sqrt{x_{*}}-\sqrt{z}\right|}^{(\sqrt{x}+\sqrt{y}) \wedge\left(\sqrt{x_{*}}+\sqrt{z}\right)} \int_{0}^{2 \pi} \Psi\left(\sqrt{2} s, \sqrt{2} Y_{*}, x, y, z\right) \mathrm{d} \theta \mathrm{d} s\right) \mathrm{d} y \mathrm{~d} z .
\end{aligned}
$$

Since $\psi$ is arbitrary, this implies $(6.7)$ by continuity.

Lemma 6.3. Let $\Phi \in C\left(\mathbb{R}_{\geq 0}^{2}\right)$. Then for any $x, y, z \geq 0$ satisfying $x_{*}:=y+z-x \geq 0$ we have

$$
\int_{|\sqrt{x}-\sqrt{z}| \vee\left|\sqrt{x_{*}}-\sqrt{y}\right|}^{(\sqrt{x}+\sqrt{z}) \wedge\left(\sqrt{x_{*}}+\sqrt{y}\right)} \mathrm{d} s \int_{0}^{2 \pi} \Phi\left(\sqrt{2} \widetilde{Y}_{*}, \sqrt{2} s\right) \mathrm{d} \theta=\int_{|\sqrt{x}-\sqrt{y}| \vee\left|\sqrt{x_{*}}-\sqrt{z}\right|}^{(\sqrt{x}+\sqrt{y}) \wedge\left(\sqrt{x_{*}}+\sqrt{z}\right)} \mathrm{d} s \int_{0}^{2 \pi} \Phi\left(\sqrt{2} s, \sqrt{2} Y_{*}\right) \mathrm{d} \theta
$$


and

$$
\begin{aligned}
& \int_{|\sqrt{x}-\sqrt{y}| \vee\left|\sqrt{x_{*}}-\sqrt{z}\right|}^{(\sqrt{x}+\sqrt{y}) \vee\left(\sqrt{x_{*}}+\sqrt{z}\right)} \mathrm{d} s \int_{0}^{2 \pi} \frac{\sqrt{z} s}{\sqrt{y} Y_{*}+\sqrt{z} s} \Phi\left(\sqrt{2} s, \sqrt{2} Y_{*}\right) \mathrm{d} \theta \\
& +\int_{|\sqrt{x}-\sqrt{z}| \vee\left|\sqrt{x_{*}}-\sqrt{y}\right|}^{(\sqrt{x}+\sqrt{z}) \wedge\left(\sqrt{x_{*}}+\sqrt{y}\right)} \mathrm{d} s \int_{0}^{2 \pi} \frac{\sqrt{y} s}{\sqrt{z} \widetilde{Y}_{*}+\sqrt{y} s} \Phi\left(\sqrt{2} \widetilde{Y}_{*}, \sqrt{2} s\right) \mathrm{d} \theta \\
& =\int_{|\sqrt{x}-\sqrt{y}| \vee\left|\sqrt{x_{*}}-\sqrt{z}\right|}^{(\sqrt{x}+\sqrt{y}) \vee\left(\sqrt{x_{*}}+\sqrt{z}\right)} \mathrm{d} s \int_{0}^{2 \pi} \Phi\left(\sqrt{2} s, \sqrt{2} Y_{*}\right) \mathrm{d} \theta .
\end{aligned}
$$

Proof. Recall $(\sqrt{x}+\sqrt{y}) \wedge\left(\sqrt{x_{*}}+\sqrt{z}\right)-|\sqrt{x}-\sqrt{y}| \vee\left|\sqrt{x_{*}}-\sqrt{z}\right|=2 \min \left\{\sqrt{x}, \sqrt{x_{*}}, \sqrt{y}, \sqrt{z}\right\}$. If $\min \left\{\sqrt{x}, \sqrt{x_{*}}, \sqrt{y}, \sqrt{z}\right\}=0$, then the above integrals are all zero.

In the following we suppose $\min \left\{\sqrt{x}, \sqrt{x_{*}}, \sqrt{y}, \sqrt{z}\right\}>0$. Take any $\psi \in C_{c}\left(\mathbb{R}_{\geq 0}^{2}\right)$. Applying the second equality in (6.4) of Lemma 6.1 to the function $\Phi\left(\left|\mathbf{v}-\mathbf{v}^{\prime}\right|,\left|\mathbf{v}-\mathbf{v}_{*}^{\prime}\right|\right) \psi\left(\left|\mathbf{v}^{\prime}\right|^{2} / 2,\left|\mathbf{v}_{*}^{\prime}\right|^{2} / 2\right)$ we have

$$
\begin{aligned}
& \int_{\mathbb{R}_{\geq 0}^{2}, y+z>x} \psi(y, z)\left(\int_{|\sqrt{x}-\sqrt{y}| \vee\left|\sqrt{x_{*}}-\sqrt{z}\right|}^{(\sqrt{x}+\sqrt{y}) \wedge\left(\sqrt{x_{*}}+\sqrt{z}\right)} \mathrm{d} s \int_{0}^{2 \pi} \Phi\left(\sqrt{2} s, \sqrt{2} Y_{*}\right) \mathrm{d} \theta\right) \mathrm{d} y \mathrm{~d} z \\
& =\int_{\mathbb{R}_{\geq 0}^{2}, y+z>x} \psi(y, z)\left(\int_{|\sqrt{x}-\sqrt{z}| \vee\left|\sqrt{x_{*}}-\sqrt{y}\right|}^{(\sqrt{x}+\sqrt{z}) \wedge\left(\sqrt{x_{*}}+\sqrt{y}\right)} \mathrm{d} s \int_{0}^{2 \pi} \Phi\left(\sqrt{2} \widetilde{Y}_{*}, \sqrt{2} s\right) \mathrm{d} \theta\right) \mathrm{d} y \mathrm{~d} z .
\end{aligned}
$$

Since $\psi \in C_{c}\left(\mathbb{R}_{\geq 0}^{2}\right)$ is arbitrary and both sides of (6.8) are continuous in $(x, y, z)$, this implies that (6.8) holds true.

To prove (6.9), we fix $x, y, z$ mentioned in the lemma and apply Lemma 6.3 to continuous functions

$$
(r, \rho) \mapsto \Phi_{n}(r, \rho)=\frac{\sqrt{2 z} r}{\sqrt{2 y} \rho+\sqrt{2 z} r+1 / n} \Phi(r, \rho), \quad(r, \rho) \in \mathbb{R}_{\geq 0}^{2}
$$

to get the revelent equality and then letting $n \rightarrow \infty$ and using Lebesgue dominated convergence we obtain the equality

$$
\begin{aligned}
& \int_{|\sqrt{x}-\sqrt{y}| \vee\left|\sqrt{x_{*}}-\sqrt{z}\right|}^{(\sqrt{x}+\sqrt{y}) \vee\left(\sqrt{x_{*}}+\sqrt{z}\right)} \mathrm{d} s \int_{0}^{2 \pi} \frac{\sqrt{z} s}{\sqrt{y} Y_{*}+\sqrt{z} s} \Phi\left(\sqrt{2} s, \sqrt{2} Y_{*}\right) \mathrm{d} \theta \\
& =\int_{|\sqrt{x}-\sqrt{z}| \vee\left|\sqrt{x_{*}}-\sqrt{y}\right|}^{(\sqrt{x}+\sqrt{z}) \vee\left(\sqrt{x_{*}}+\sqrt{y}\right)} \mathrm{d} s \int_{0}^{2 \pi} \frac{\sqrt{z} \widetilde{Y}_{*}}{\sqrt{y} s+\sqrt{z} \widetilde{Y}_{*}} \Phi\left(\sqrt{2} \widetilde{Y}_{*}, \sqrt{2} s\right) \mathrm{d} \theta .
\end{aligned}
$$

From this and (6.8) it is easily deduced (6.9).

Remark 6.4. Applying (6.8) to the function $\Phi(r, \rho)$ given in (1.5) one sees that the function $W(x, y, z)$ (defined in $(1.19),(1.20))$ is symmetric in $y, z$, i.e. $W(x, y, z) \equiv W(x, z, y)$ on $\mathbb{R}_{\geq 0}^{3}$.

The following lemma deals with equalities for total integration where the integrand $\Psi$ can be arbitrary nonnegative Lebesgue measurable function. 
Lemma 6.5. Let $0 \leq \Phi \in C_{b}\left(\mathbb{R}_{\geq 0}^{2}\right), \Psi: \mathbb{R}_{\geq 0}^{3} \rightarrow \mathbb{R}_{\geq 0}$ be Lebesgue measurable. Then

$$
\begin{aligned}
& \int_{\mathbb{R}^{3} \times \mathbb{R}^{3} \times \mathbb{S}^{2}} \frac{\left|\left(\mathbf{v}-\mathbf{v}_{*}\right) \cdot \omega\right|}{(4 \pi)^{2}} \Phi\left(\left|\mathbf{v}-\mathbf{v}^{\prime}\right|,\left|\mathbf{v}-\mathbf{v}_{*}^{\prime}\right|\right) \Psi\left(|\mathbf{v}|^{2} / 2,\left|\mathbf{v}^{\prime}\right|^{2} / 2,\left|\mathbf{v}_{*}^{\prime}\right|^{2} / 2\right) \mathrm{d} \omega \mathrm{d} \mathbf{v}_{*} \mathrm{~d} \mathbf{v} \\
& =\sqrt{2} \int_{\mathbb{R}_{\geq 0}^{3}} 1_{\{y+z>x\}} \Psi(x, y, z)\left(\int_{|\sqrt{x}-\sqrt{y}| \vee\left|\sqrt{x_{*}}-\sqrt{z}\right|}^{(\sqrt{x}+\sqrt{y}) \wedge\left(\sqrt{x_{*}}+\sqrt{z}\right)} \int_{0}^{2 \pi} \Phi\left(\sqrt{2} s, \sqrt{2} Y_{*}\right) \mathrm{d} \theta \mathrm{d} s\right) \mathrm{d} x \mathrm{~d} y \mathrm{~d} z \\
& =\sqrt{2} \int_{\mathbb{R}_{\geq 0}^{3}} 1_{\{y+z>x\}} \Psi(x, y, z)\left(\int_{|\sqrt{x}-\sqrt{z}| \vee\left|\sqrt{x_{*}}-\sqrt{y}\right|}^{(\sqrt{x}+\sqrt{z}) \wedge\left(\sqrt{x_{*}}+\sqrt{y}\right)} \int_{0}^{2 \pi} \Phi\left(\sqrt{2} \widetilde{Y}_{*}, \sqrt{2} s\right) \mathrm{d} \theta \mathrm{d} s\right) \mathrm{d} x \mathrm{~d} y \mathrm{~d} z .(6.10)
\end{aligned}
$$

Proof. We need only prove the first equality sign in (6.10) since, by Lemma 6.3, the second equality holds true. [In fact, using the same proof below one sees that the first line in (6.10) also equals to the third line.] For notation convenience we define the measure $\mu$ on $\mathbb{R}^{3} \times \mathbb{S}^{2}$ by

$$
\mathrm{d} \mu\left(\mathbf{v}_{*}, \omega\right)=\frac{\left|\left(\mathbf{v}-\mathbf{v}_{*}\right) \cdot \omega\right|}{(4 \pi)^{2}} \Phi\left(\left|\mathbf{v}-\mathbf{v}^{\prime}\right|,\left|\mathbf{v}-\mathbf{v}_{*}^{\prime}\right|\right) \mathrm{d} \omega \mathrm{d} \mathbf{v}_{*}
$$

and let

$$
\varrho(x, y, z)=\sqrt{2} \cdot 1_{\{y+z>x\}} \int_{|\sqrt{x}-\sqrt{y}| \vee\left|\sqrt{x_{*}}-\sqrt{z}\right|}^{(\sqrt{x}+\sqrt{y}) \wedge\left(\sqrt{x_{*}}+\sqrt{z}\right)} \int_{0}^{2 \pi} \Phi\left(\sqrt{2} s, \sqrt{2} Y_{*}\right) \mathrm{d} \theta \mathrm{d} s, \quad(x, y, z) \in \mathbb{R}_{\geq 0}^{3} .
$$

Then the first equality in (6.10) is written

$$
\int_{\mathbb{R}^{3} \times \mathbb{R}^{3} \times \mathbb{S}^{2}} \Psi\left(|\mathbf{v}|^{2} / 2,\left|\mathbf{v}^{\prime}\right|^{2} / 2,\left|\mathbf{v}_{*}^{\prime}\right|^{2} / 2\right) \mathrm{d} \mu\left(\mathbf{v}_{*}, \omega\right) \mathrm{d} \mathbf{v}=\int_{\mathbb{R}_{\geq 0}^{3}} \Psi(x, y, z) \varrho(x, y, z) \mathrm{d} x \mathrm{~d} y \mathrm{~d} z .
$$

From (1.23) we have

$$
0 \leq \varrho(x, y, z) \leq \sqrt{2} \cdot 4 \pi\|\Phi\|_{\infty} 1_{\{y+z>x\}} \min \left\{\sqrt{x}, \sqrt{x_{*}}, \sqrt{y}, \sqrt{z}\right\} \quad \forall(x, y, z) \in \mathbb{R}_{\geq 0}^{3}
$$

Then it is easily proved that $\varrho$ is continuous on $\mathbb{R}_{\geq 0}^{3}$. But in the present proof, we need only the inequality (6.12) and the fact that $\varrho$ is Lebesgue measurable on $\mathbb{R}_{\geq 0}^{3}$. Now let us prove (6.11).

Step1. We prove that if $0 \leq \Psi \in C\left(\mathbb{R}_{\geq 0}^{3}\right)$, then (6.11) holds true. In fact, starting from the left hand side of (6.11), using Fubini theorem and changing variable $\mathbf{v}=\sqrt{2 x} \widetilde{\omega}, \widetilde{\omega} \in \mathbb{S}^{2}$, and then applying Lemma 6.1 to the nonnegative continuous function $\left(x_{1}, x_{2}, x_{3}, x_{4}, x_{5}\right) \mapsto$ $\Phi\left(x_{1}, x_{2}\right) \Psi\left(x_{3}, x_{4}, x_{5}\right)$, one obtains the equality (6.11).

Step2. Suppose that $\Psi$ is Lebesgue measurable on $\mathbb{R}_{\geq 0}^{3}$ satisfying

$$
0 \leq \Psi(x, y, z)<M \quad \text { and } \quad 0 \leq \Psi(x, y, z) \leq M 1_{[0, R]^{3}}(x, y, z) \quad \forall(x, y, z) \in \mathbb{R}_{\geq 0}^{3}
$$

for some constants $0<M, R<\infty$. In this case we prove that (6.11) holds true.

In fact for any $n \in \mathbb{N}$, applying Lusin's theorem (see e.g. a proof in [28]) there exists a function $\Psi_{n} \in C\left(\mathbb{R}^{3}\right)$ satisfying

$$
0 \leq \Psi_{n}(x, y, z)<M \quad \forall(x, y, z) \in \mathbb{R}^{3} ; \quad \operatorname{supp} \Psi_{n} \subset[-1 / n, R+1 / n]^{3}
$$


such that

$$
\operatorname{mes}\left(\left\{(x, y, z) \in[0, R]^{3} \mid \Psi(x, y, z) \neq \Psi_{n}(x, y, z)\right\}\right)<\frac{1}{n} .
$$

Then it is easily seen that $\Psi_{n} \rightarrow \Psi(n \rightarrow \infty)$ in $L^{1}\left(\mathbb{R}_{\geq 0}^{3}\right)$. So there is a subsequence $\left\{\Psi_{n_{k}}\right\}_{k=1}^{\infty}$ and a null set $Z \subset \mathbb{R}_{\geq 0}^{3}$ such that $\Psi_{n_{k}}(x, y, z) \rightarrow \Psi(x, y, z)(k \rightarrow \infty)$ for all $(x, y, z) \in \mathbb{R}_{\geq 0} \backslash Z$. Note that from the bound in (6.12) we also have the weighted strong convergence: $\Psi_{n} \rightarrow \Psi(n \rightarrow \infty)$ in $L^{1}\left(\mathbb{R}_{\geq 0}^{3}, \varrho(x, y, z) \mathrm{d} x \mathrm{~d} y \mathrm{~d} z\right)$. It is easily proved that the set

$$
\widetilde{Z}=\left\{\left(\mathbf{v}, \mathbf{v}_{*}, \omega\right) \in \mathbb{R}^{3} \times \mathbb{R}^{3} \times \mathbb{S}^{2} \mid\left(|\mathbf{v}|^{2} / 2,\left|\mathbf{v}^{\prime}\right|^{2} / 2,\left|\mathbf{v}_{*}^{\prime}\right|^{2} / 2\right) \in Z\right\}
$$

has measure zero with the measure $\mathrm{d} \mu\left(\mathbf{v}_{*}, \omega\right) \mathrm{d} \mathbf{v}$ (here as usual we may assume that the product measure $\mathrm{d} \mu\left(\mathbf{v}_{*}, \omega\right) \mathrm{d} \mathbf{v}$ is a complete measure). Thus $\Psi_{n_{k}}\left(|\mathbf{v}|^{2} / 2,\left|\mathbf{v}^{\prime}\right|^{2} / 2,\left|\mathbf{v}_{*}^{\prime}\right|^{2} / 2\right)$ $\rightarrow \Psi\left(|\mathbf{v}|^{2} / 2,\left|\mathbf{v}^{\prime}\right|^{2} / 2,\left|\mathbf{v}_{*}^{\prime}\right|^{2} / 2\right)(k \rightarrow \infty)$ for all $\left(\mathbf{v}, \mathbf{v}_{*}, \omega\right) \in \mathbb{R}^{3} \times \mathbb{R}^{3} \times \mathbb{S}^{2} \backslash \widetilde{Z}$. Since, by Step1, $\Psi_{n_{k}}$ satisfy (6.11), it follows from Fatou's lemma that

$$
\int_{\mathbb{R}^{3} \times \mathbb{R}^{3} \times \mathbb{S}^{2}} \Psi\left(|\mathbf{v}|^{2} / 2,\left|\mathbf{v}^{\prime}\right|^{2} / 2,\left|\mathbf{v}_{*}^{\prime}\right|^{2} / 2\right) \mathrm{d} \mu\left(\mathbf{v}_{*}, \omega\right) \mathrm{d} \mathbf{v} \leq \int_{\mathbb{R}_{\geq 0}^{3}} \Psi(x, y, z) \varrho(x, y, z) \mathrm{d} x \mathrm{~d} y \mathrm{~d} z .
$$

Since $\left|\Psi-\Psi_{n}\right|$ have the same properties as $\Psi$ (just by replacing $R$ with $R+1$ ), the inequality (6.13) holds also for $\left|\Psi-\Psi_{n}\right|$ and thus $\Psi_{n} \rightarrow \Psi(n \rightarrow \infty)$ in $L^{1}\left(\mathbb{R}^{3} \times \mathbb{R}^{3} \times \mathbb{S}^{2}, \mathrm{~d} \mu\left(\mathbf{v}_{*}, \omega\right) \mathrm{d} \mathbf{v}\right)$. We then conclude that $\Psi$ satisfies the equality (6.11).

Step3. Let $\Psi$ be given in the lemma and let

$$
\Psi_{n}(x, y, z)=\Psi(x, y, z) 1_{\{\Psi(x, y, z)<n\}} 1_{[0, n]^{3}}(x, y, z), \quad(x, y, z) \in \mathbb{R}_{\geq 0}^{3}, n \in \mathbb{N} .
$$

Then $\Psi_{n}$ satisfy the conditions in Step2 with $M=R=n$. So (6.11) holds for all $\Psi_{n}$. Also we have $0 \leq \Psi_{n} \leq \Psi_{n+1}$ and $\lim _{n \rightarrow \infty} \Psi_{n}(x, y, z)=\Psi(x, y, z)$ for all $(x, y, z) \in \mathbb{R}_{\geq 0}^{3}$. Thus taking the limit $n \rightarrow \infty$ to (6.11) for $\Psi_{n}$ we conclude from Levi's monotone convergence theorem that (6.11) holds true for $\Psi$.

6.2. Equivalence of Solutions. In [19] the measure-valued isotropic solution is defined for the measure $\bar{F} \in \mathcal{B}_{2}^{+}\left(\mathbb{R}_{\geq 0}\right)$ whose special case is $\mathrm{d} \bar{F}(r)=4 \pi r^{2} f\left(r^{2} / 2\right) \mathrm{d} r$, and the corresponding integrands $J_{B}[\varphi]\left(r, r_{*}\right), K_{B}[\varphi]\left(r, r^{\prime}, r_{*}^{\prime}\right)$ for quadratic and cubic collision integrals are defined as follows: denote

$$
B\left(\left|\mathbf{v}-\mathbf{v}_{*}\right|, \cos (\theta)\right)=\frac{\left|\mathbf{v}-\mathbf{v}_{*}\right| \cos (\theta)}{(4 \pi)^{2}} \Phi\left(\left|\mathbf{v}-\mathbf{v}_{*}\right| \cos (\theta),\left|\mathbf{v}-\mathbf{v}_{*}\right| \sin (\theta)\right)
$$

$\theta=\arccos \left(\left|\left(\mathbf{v}-\mathbf{v}_{*}\right) \cdot \omega\right| /\left|\mathbf{v}-\mathbf{v}_{*}\right|\right)$, and for any $\varphi \in C_{b}^{2}\left(\mathbb{R}_{\geq 0}\right)$, let

$$
\begin{aligned}
J_{B}[\varphi]\left(r, r_{*}\right) & =\frac{2}{(4 \pi)^{2}} \int_{\mathbb{S}^{2} \times \mathbb{S}^{2}} \mathrm{~d} \sigma \mathrm{d} \sigma_{*} \int_{0}^{\pi / 2} B\left(\left|\mathbf{v}-\mathbf{v}_{*}\right|, \cos (\theta)\right) \sin (\theta) \mathrm{d} \theta \\
& \times\left.\int_{0}^{2 \pi}\left(\varphi\left(\left|\mathbf{v}^{\prime}\right|^{2} / 2\right)-\varphi\left(|\mathbf{v}|^{2} / 2\right)\right) \mathrm{d} \vartheta\right|_{\mathbf{v}=r \sigma, \mathbf{v}_{*}=r_{*} \sigma_{*}}
\end{aligned}
$$




$$
\begin{aligned}
& K_{B}[\varphi]\left(r, r^{\prime}, r_{*}^{\prime}\right)=\frac{4}{(4 \pi)^{3}} \int_{\mathbb{S}^{2} \times \mathbb{S}^{2}} \mathrm{~d} \sigma \mathrm{d} \sigma^{\prime} \int_{0}^{2 \pi} 1_{\left\{r_{*}^{\prime}>r|\sigma \cdot \xi|\right\}} \frac{\Delta \varphi\left(r^{2} / 2, r^{\prime 2} / 2, r_{*}^{\prime 2} / 2\right)}{X r_{*}^{\prime}+Y r^{\prime}} \Phi(X, Y) \mathrm{d} \vartheta \\
& \text { if } \quad\left(r^{\prime}-r\right)\left(r_{*}^{\prime}-r\right) \neq 0, \\
& K_{B}[\varphi]\left(r, r^{\prime}, r_{*}^{\prime}\right)=0 \text { if }\left(r^{\prime}-r\right)\left(r_{*}^{\prime}-r\right)=0
\end{aligned}
$$

where $\left(\mathbf{v}^{\prime}, \mathbf{v}_{*}^{\prime}\right)$ is given by the $\omega$-representation $(1.2)$,

$$
\begin{aligned}
& \left|\mathbf{v}^{\prime}\right|^{2}=r^{2} \sin ^{2}(\theta)+r_{*}^{2} \cos ^{2}(\theta)-2 r r_{*} \sin (\theta) \cos (\theta) \sqrt{1-\left\langle\sigma, \sigma_{*}\right\rangle} \cos (\vartheta), \\
& \left|\mathbf{v}_{*}^{\prime}\right|^{2}=r^{2} \cos ^{2}(\theta)+r_{*}^{2} \sin ^{2}(\theta)+2 r r_{*} \sin (\theta) \cos (\theta) \sqrt{1-\left\langle\sigma, \sigma_{*}\right\rangle} \cos (\vartheta), \\
& \Delta \varphi\left(r^{2} / 2, r^{\prime 2} / 2, r_{*}^{\prime 2} / 2\right)=\varphi\left(r^{2} / 2\right)+\varphi\left(r_{*}^{2} / 2\right)-\varphi\left(r^{\prime 2} / 2\right)-\varphi\left(r_{*}^{\prime 2} / 2\right), \\
& r_{*}=\sqrt{\left(r^{\prime 2}+r_{*}^{\prime 2}-r^{2}\right)_{+}}, \\
& X=\left|r \sigma-r^{\prime} \sigma^{\prime}\right|, \quad Y=\left|\sqrt{r_{*}^{\prime 2}-|\langle r \sigma, \xi\rangle|^{2}}+e^{\mathrm{i} \vartheta} \sqrt{r^{2}-\langle r \sigma, \xi\rangle^{2}}\right|, \quad r_{*}^{\prime}>|r \sigma \cdot \xi|, \\
& \xi=\frac{r \sigma-r^{\prime} \sigma^{\prime}}{\left|r \sigma-r^{\prime} \sigma^{\prime}\right|} \text { if } r \sigma \neq r^{\prime} \sigma^{\prime} ; \quad \xi=\sigma \text { if } r \sigma=r^{\prime} \sigma^{\prime} .
\end{aligned}
$$

Note that $r_{*}^{\prime}>|r \sigma \cdot \xi|$ implies ${r^{\prime 2}}^{2}+{r_{*}^{\prime}}^{2}>r^{2}$, and using (1.18) we have

$$
\left|\Delta \varphi\left(r^{2} / 2, r^{\prime 2} / 2, r_{*}^{\prime 2} / 2\right)\right| \leq \frac{1}{4}\left\|\varphi^{\prime \prime}\right\|_{\infty}\left|\left(r^{\prime 2}-r^{2}\right)\left({r_{*}^{\prime}}^{2}-r^{2}\right)\right|
$$

from which one sees that if $\left(r^{\prime}-r\right)\left(r_{*}^{\prime}-r\right) \neq 0$ and $r_{*}^{\prime}>r|\sigma \cdot \xi|$ then $X \geq\left|r^{\prime}-r\right|>0, Y \geq$ $\left|r_{*}^{\prime}-r\right|>0$ and so $K_{B}[\varphi]\left(r, r^{\prime}, r_{*}^{\prime}\right)$ is well defined on $\mathbb{R}_{\geq 0}^{3}$. Also it has been proven in [19] that $J_{B}[\varphi], K_{B}[\varphi]$ are continuous on $\mathbb{R}_{\geq 0}^{2}, \mathbb{R}_{\geq 0}^{3}$ respectively.

Definition 6.6. ([19]) Let $B\left(\mathbf{v}-\mathbf{v}_{*}, \omega\right)$ be given by (1.4), (1.5). Let $\bar{F}_{0} \in \mathcal{B}_{2}^{+}\left(\mathbb{R}_{\geq 0}\right)$. We say that a family $\left\{\bar{F}_{t}\right\}_{t \geq 0} \subset \mathcal{B}_{2}^{+}\left(\mathbb{R}_{\geq 0}\right)$, or simply $\bar{F}_{t}$, is a conservative measure-valued isotropic solution of Eq.(1.1) on the time-interval $[0, \infty)$ with the initial datum $\left.\bar{F}_{t}\right|_{t=0}=\bar{F}_{0}$ if

(i) $\int_{\mathbb{R}_{\geq 0}}\left(1, r^{2}\right) \mathrm{d} \bar{F}_{t}(r)=\int_{\mathbb{R}_{\geq 0}}\left(1, r^{2}\right) \mathrm{d} \bar{F}_{0}(r)$ for all $t \in[0, \infty)$,

(ii) for every $\varphi \in C_{b}^{2}\left(\mathbb{R}_{\geq 0}\right), t \mapsto \int_{\mathbb{R}_{\geq 0}} \varphi\left(r^{2} / 2\right) \mathrm{d} \bar{F}_{t}(r)$ belongs to $C^{1}([0, \infty))$,

(iii) for every $\varphi \in C_{b}^{2}\left(\mathbb{R}_{\geq 0}\right)$ and $t \in[0, \infty)$,

$$
\begin{aligned}
& \frac{\mathrm{d}}{\mathrm{d} t} \int_{\mathbb{R}_{\geq 0}} \varphi\left(r^{2} / 2\right) \mathrm{d} \bar{F}_{t}(r) \\
& =\int_{\mathbb{R}_{\geq 0}^{2}} J_{B}[\varphi]\left(r, r_{*}\right) \mathrm{d} \bar{F}_{t}(r) \mathrm{d} \bar{F}_{t}\left(r_{*}\right)+\int_{\mathbb{R}_{\geq 0}^{3}} K_{B}[\varphi]\left(r, r^{\prime}, r_{*}^{\prime}\right) \mathrm{d} \bar{F}_{t}(r) \mathrm{d} \bar{F}_{t}\left(r^{\prime}\right) \mathrm{d} \bar{F}_{t}\left(r_{*}^{\prime}\right) .
\end{aligned}
$$

It has been proven in [19] that for any $\bar{F}_{0} \in \mathcal{B}_{2}^{+}\left(\mathbb{R}_{\geq 0}\right)$, the Eq.(1.1) with the initial datum $\left.\bar{F}\right|_{t=0}=\bar{F}_{0}$ has a conservative measure-valued isotropic solution on $[0, \infty)$ in the sense of Definition6.6.

Let $F \in \mathcal{B}_{1}^{+}\left(\mathbb{R}_{\geq 0}\right), \bar{F} \in \mathcal{B}_{2}^{+}\left(\mathbb{R}_{\geq 0}\right)$ be defined from each other by

$$
F(A)=\frac{1}{4 \pi \sqrt{2}} \int_{\mathbb{R}_{\geq 0}} \mathbf{1}_{A}\left(r^{2} / 2\right) \mathrm{d} \bar{F}(r), \quad \bar{F}(A)=4 \pi \sqrt{2} \int_{\mathbb{R}_{\geq 0}} \mathbf{1}_{A}(\sqrt{2 x}) \mathrm{d} F(x)
$$


for all Borel sets $A \subset \mathbb{R}_{\geq 0}$. It is easily seen that if $F \in \mathcal{B}_{1}^{+}\left(\mathbb{R}_{\geq 0}\right), \bar{F} \in \mathcal{B}_{2}^{+}\left(\mathbb{R}_{\geq 0}\right)$ are given through one of the equalities in (6.16), then

$$
4 \pi \sqrt{2} \int_{\mathbb{R}_{\geq 0}} \varphi(x) \mathrm{d} F(x)=\int_{\mathbb{R}_{\geq 0}} \varphi\left(r^{2} / 2\right) \mathrm{d} \bar{F}(r)
$$

for all Borel measurable functions $\varphi$ on $\mathbb{R}_{\geq 0}$ satisfying $\sup (1+x)^{-1}|\varphi(x)|<\infty$. In the special case where $F$ and $\bar{F}$ are given by $\mathrm{d} F(x)=f(x) \sqrt{x} \mathrm{~d} x, \mathrm{~d} \bar{F}(r)=4 \pi f\left(r^{2} / 2\right) r^{2} \mathrm{~d} r$, the above relation is just the change of variable.

The following lemma and proposition show that the Definition 1.2 and Definition 6.6 are equivalent. This also ensures the existence of solutions in the sense of Definition 1.2.

Lemma 6.7. Let $B\left(\mathbf{v}-\mathbf{v}_{*}, \omega\right)$ be given by (1.4), (1.5), and let $\bar{F} \in \mathcal{B}_{2}^{+}\left(\mathbb{R}_{\geq 0}\right), F \in \mathcal{B}_{1}^{+}\left(\mathbb{R}_{\geq 0}\right)$ be given through one of the equalities in (6.16). Then for any $\varphi \in C_{b}^{2}\left(\mathbb{R}_{\geq 0}\right)$ we have

$$
\begin{array}{r}
\int_{\mathbb{R}_{\geq 0}^{2}} J_{B}[\varphi]\left(r, r_{*}\right) \mathrm{d} \bar{F}(r) \mathrm{d} \bar{F}\left(r_{*}\right)=4 \pi \sqrt{2} \int_{\mathbb{R}_{\geq 0}^{2}} \mathcal{J}[\varphi](y, z) \mathrm{d} F(y) \mathrm{d} F(z), \\
\int_{\mathbb{R}_{\geq 0}^{3}} K_{B}[\varphi]\left(r, r^{\prime}, r_{*}^{\prime}\right) \mathrm{d} \bar{F}(r) \mathrm{d} \bar{F}\left(r^{\prime}\right) \mathrm{d} \bar{F}\left(r_{*}^{\prime}\right)=4 \pi \sqrt{2} \int_{\mathbb{R}_{\geq 0}^{3}} \mathcal{K}[\varphi](x, y, z) \mathrm{d} F(x) \mathrm{d} F(y) \mathrm{d} F(z) .
\end{array}
$$

Proposition 6.8. Let $F_{t} \in \mathcal{B}_{1}^{+}\left(\mathbb{R}_{\geq 0}\right), \bar{F}_{t} \in \mathcal{B}_{2}^{+}\left(\mathbb{R}_{\geq 0}\right)$ satisfy one of the equalities in (6.16) for every $t \in[0, \infty)$. Then $F_{t}$ is a conservative measure-valued isotropic solution of Eq.(1.1) in the sense of Definition 1.2, if and only if $\bar{F}_{t}$ is a conservative measure-valued isotropic solution of Eq.(1.1) in the sense of Definition 6.6.

Proof of Lemma 6.7 and Proposition 6.8. From (6.17) we see that Proposition 6.8 follows easily from Lemma 6.7. So we need only prove Lemma 6.7.

We first prove that for any $\varphi \in C_{c}^{2}(\mathbb{R})$ and any $\left(r, r_{*}\right) \in \mathbb{R}_{\geq 0}^{2}$

$$
\begin{aligned}
& \int_{\mathbb{S}^{2} \times \mathbb{S}^{2}} \mathrm{~d} \sigma \mathrm{d} \sigma_{*} \int_{\mathbb{S}^{2}} \frac{\left|\left(\mathbf{v}-\mathbf{v}_{*}\right) \cdot \omega\right|}{(4 \pi)^{2}} \Phi\left(\left|\mathbf{v}-\mathbf{v}^{\prime}\right|,\left|\mathbf{v}-\mathbf{v}_{*}^{\prime}\right|\right) \\
& \times\left.\left(\varphi\left(\left|\mathbf{v}^{\prime}\right|^{2} / 2\right)+\varphi\left(\left|\mathbf{v}_{*}^{\prime}\right|^{2} / 2\right)-\varphi\left(|\mathbf{v}|^{2} / 2\right)-\varphi\left(\left|\mathbf{v}_{*}\right|^{2} / 2\right)\right)\right|_{\mathbf{v}=r \sigma, \mathbf{v}_{*}=r_{*} \sigma_{*}} \mathrm{~d} \omega \\
& =4 \pi \sqrt{2} \mathcal{J}[\varphi]\left(r^{2} / 2, r_{*}^{2} / 2\right) .
\end{aligned}
$$

It is easily seen that the right hand side of (6.20) is also continuous in $\left(r, r_{*}\right) \in \mathbb{R}_{\geq 0}^{2}$. Thus we need only prove $(6.20)$ for all $\left(r, r_{*}\right) \in \mathbb{R}_{>0}^{2}$. Let $\left(r, r_{*}\right) \in \mathbb{R}_{>0}^{2}$. Using Lemma 6.2 to $y=r^{2} / 2, z=r_{*}^{2} / 2$ and recalling definition of $\mathcal{J}[\varphi]$ we have

$$
\begin{aligned}
& \text { the 1.h.s. of }(6.20)=\frac{1}{\sqrt{2 y z}} \int_{0}^{y+z} \mathrm{~d} x \int_{|\sqrt{y}-\sqrt{x}| \vee\left|\sqrt{x_{*}}-\sqrt{z}\right|}^{(\sqrt{y}+\sqrt{x}) \wedge\left(\sqrt{z}+\sqrt{x_{*}}\right)} \mathrm{d} s \int_{0}^{2 \pi} \Phi\left(\sqrt{2} s, \sqrt{2} Y_{*}\right) \\
& \times\left.\left(\varphi\left(x_{*}\right)+\varphi(x)-\varphi(z)-\varphi(y)\right) \mathrm{d} \theta\right|_{\mathbf{v}=\sqrt{2 z} \sigma, \mathbf{v}_{*}=\sqrt{2 y} \sigma_{*}} \\
& =4 \pi \sqrt{2} \mathcal{J}[\varphi](y, z)=4 \pi \sqrt{2} \mathcal{J}[\varphi]\left(r^{2} / 2, r_{*}^{2} / 2\right) .
\end{aligned}
$$


Now first making exchanges $r \leftrightarrow r_{*}, \sigma \leftrightarrow \sigma_{*}$ and using $\int_{0}^{2 \pi} g(\cos \vartheta) \mathrm{d} \vartheta=\int_{0}^{2 \pi} g(-\cos \vartheta) \mathrm{d} \theta$ and then using (6.20) we compute

$$
\begin{aligned}
& \int_{\mathbb{R}_{\geq 0}^{2}} J_{B}[\varphi]\left(r, r_{*}\right) \mathrm{d} \bar{F}(r) \mathrm{d} \bar{F}\left(r_{*}\right) \\
& =\frac{1}{(4 \pi)^{2}} \int_{\mathbb{R}_{\geq 0}^{2}} \int_{\mathbb{S}^{2} \times \mathbb{S}^{2}} \mathrm{~d} \sigma \mathrm{d} \sigma_{*} \int_{0}^{\pi / 2} B\left(\left|\mathbf{v}-\mathbf{v}_{*}\right|, \cos (\theta)\right) \sin (\theta) \mathrm{d} \theta \\
& \times\left.\int_{0}^{2 \pi}\left(\varphi\left(\left|\mathbf{v}^{\prime}\right|^{2} / 2\right)+\varphi\left(\left|\mathbf{v}_{*}^{\prime}\right|^{2} / 2\right)-\varphi\left(|\mathbf{v}|^{2} / 2\right)-\varphi\left(\left|\mathbf{v}_{*}\right|^{2} / 2\right)\right) \mathrm{d} \vartheta\right|_{\mathbf{v}=r \sigma, \mathbf{v}_{*}=r_{*} \sigma_{*}} \mathrm{~d} \bar{F}(r) \mathrm{d} \bar{F}\left(r_{*}\right) \\
& =\frac{1}{(4 \pi)^{2}} \frac{1}{2} \int_{\mathbb{R}_{\geq 0}^{2}} \int_{\mathbb{S}^{2} \times \mathbb{S}^{2}} \mathrm{~d} \sigma \mathrm{d} \sigma_{*} \int_{\mathbb{S}^{2}} \frac{\left|\left(\mathbf{v}-\mathbf{v}_{*}\right) \cdot \omega\right|}{(4 \pi)^{2}} \Phi\left(\left|\mathbf{v}-\mathbf{v}^{\prime}\right|,\left|\mathbf{v}-\mathbf{v}_{*}^{\prime}\right|\right) \\
& \times\left(\varphi\left(\left|\mathbf{v}^{\prime}\right|^{2} / 2\right)+\varphi\left(\left|\mathbf{v}_{*}^{\prime}\right|^{2} / 2\right)-\left.\varphi\left(|\mathbf{v}|^{2} / 2-\varphi\left(\left|\mathbf{v}_{*}\right|^{2} / 2\right)\right) \mathrm{d} \omega\right|_{\mathbf{v}=r \sigma, \mathbf{v}_{*}=r_{*} \sigma_{*}} \mathrm{~d} \bar{F}(r) \mathrm{d} \bar{F}\left(r_{*}\right)\right. \\
& =\frac{1}{(4 \pi)^{2}} \frac{1}{2} 4 \pi \sqrt{2} \int_{\mathbb{R}_{\geq 0}^{2}} \mathcal{J}[\varphi]\left(r^{2} / 2, r_{*}^{2} / 2\right) \mathrm{d} \bar{F}(r) \mathrm{d} \bar{F}\left(r_{*}\right) \\
& =4 \pi \sqrt{2} \int_{\mathbb{R}_{\geq 0}^{2}} \mathcal{J}[\varphi](y, z) \mathrm{d} F(y) \mathrm{d} F(z) .
\end{aligned}
$$

Next by definition of $K_{B}[\varphi]\left(r, r^{\prime}, r_{*}^{\prime}\right)$ and its properties mentioned above we see that for any $r \geq 0, r^{\prime} \geq 0, r_{*}^{\prime} \geq 0$ satisfying $\left(r^{\prime}-r\right)\left(r_{*}^{\prime}-r\right)=0$, or ${r^{\prime}}^{2}+{r_{*}^{\prime}}^{2}-r^{2} \leq 0$, or $r_{*}^{\prime}=0$, then $K_{B}[\varphi]\left(r, r^{\prime}, r_{*}^{\prime}\right)=0$. So we need only consider the integration domain

$$
\mathcal{R}=\left\{\left(r, r^{\prime}, r_{*}^{\prime}\right) \in \mathbb{R}_{\geq 0} \mid\left(r^{\prime}-r\right)\left(r_{*}^{\prime}-r\right) \neq 0 \text { and }{r^{\prime}}^{2}+{r_{*}^{\prime}}^{2}-r^{2}>0 \text { and } r_{*}^{\prime}>0\right\}
$$

Then we have

$$
\begin{aligned}
& \int_{\mathbb{R}_{\geq 0}^{3}} K_{B}[\varphi]\left(r, r^{\prime}, r_{*}^{\prime}\right) \mathrm{d} \bar{F}(r) \mathrm{d} \bar{F}\left(r^{\prime}\right) \mathrm{d} \bar{F}\left(r_{*}^{\prime}\right)=\int_{\mathcal{R}} K_{B}[\varphi]\left(r, r^{\prime}, r_{*}^{\prime}\right) \mathrm{d} \bar{F}(r) \mathrm{d} \bar{F}\left(r^{\prime}\right) \mathrm{d} \bar{F}\left(r_{*}^{\prime}\right) \\
& =\left(\int_{\mathcal{R}_{1}}+\int_{\mathcal{R}_{2}}+\int_{\mathcal{R}_{3}}\right) K_{B}[\varphi]\left(r, r^{\prime}, r_{*}^{\prime}\right) \mathrm{d} \bar{F}(r) \mathrm{d} \bar{F}\left(r^{\prime}\right) \mathrm{d} \bar{F}\left(r_{*}^{\prime}\right):=I_{1}+I_{2}+I_{3}
\end{aligned}
$$

where $\mathcal{R}_{1}=\mathcal{R} \cap\left\{r>0, r^{\prime}>0\right\}, \mathcal{R}_{2}=\mathcal{R} \cap\left\{r=0, r^{\prime}>0\right\}, \mathcal{R}_{3}=\mathcal{R} \cap\left\{r>0, r^{\prime}=0\right\}$.

For the integrand $K_{B}[\varphi]\left(r, r^{\prime}, r_{*}^{\prime}\right)$ in the first term $I_{1}$ we compute using change of variables (e.g. $t=\frac{r^{2}+r^{\prime 2}-s^{2}}{2 r r^{\prime}}$ ) and then letting $x=r^{2} / 2, y=r^{\prime 2} / 2, z=r_{*}^{\prime 2} / 2\left(\right.$ for $\left.\left(r, r^{\prime}, r_{*}^{\prime}\right) \in \mathcal{R}_{1}\right)$ that

$$
\begin{aligned}
& K_{B}[\varphi]\left(r, r^{\prime}, r_{*}^{\prime}\right) \\
& =\frac{2}{4 \pi} \int_{-1}^{1} \mathrm{~d} t \int_{0}^{2 \pi} 1_{\left\{r_{*}^{\prime}>\frac{\left|r^{2}-r r^{\prime}\right|}{\sqrt{r^{2}+r^{\prime 2}-2 r r^{\prime} t}}\right\}} \frac{\Delta \varphi\left(r^{2} / 2, r^{\prime 2} / 2, r_{*}^{\prime 2} / 2\right)}{\sqrt{r^{2}+r^{\prime 2}-2 r r^{\prime} t r_{*}^{\prime}+Y r^{\prime}}} \Phi\left(\sqrt{r^{2}+r^{\prime 2}-2 r r^{\prime} t}, Y\right) \mathrm{d} \theta \\
& =\frac{2}{4 \pi} \int_{\left|r-r^{\prime}\right|}^{r+r^{\prime}} \frac{s}{r r^{\prime}} \mathrm{d} s \int_{0}^{2 \pi} 1_{\left\{r_{*}^{\prime}>\frac{\left|r^{2}-r^{\prime 2}+s^{2}\right|}{2 s}\right\}} \frac{\Delta \varphi\left(r^{2} / 2, r^{\prime 2} / 2, r_{*}^{\prime 2} / 2\right)}{s r_{*}^{\prime}+Y r^{\prime}} \Phi(s, Y) \mathrm{d} \theta \\
& =\frac{1}{4 \pi} \frac{\Delta \varphi(x, y, z)}{\sqrt{x y z}} \int_{|\sqrt{x}-\sqrt{y}| \vee|\sqrt{x *}-\sqrt{z}|}^{(\sqrt{x}+\sqrt{y}) \wedge(\sqrt{x *}+\sqrt{z})} \mathrm{d} s \int_{0}^{2 \pi} \frac{s \sqrt{z}}{s \sqrt{z}+Y_{*} \sqrt{y}} \Phi\left(\sqrt{2} s, \sqrt{2} Y_{*}\right) \mathrm{d} \theta .
\end{aligned}
$$


Then with exchange $y \leftrightarrow z$ and recalling $\Phi(r, \rho) \equiv \Phi(\rho, r)$, and using Lemma 6.3 we have

$$
\begin{aligned}
& I_{1}=(4 \pi \sqrt{2})^{3} \int_{\mathbb{R}_{\geq 0}^{3}} \mathbf{1}_{\mathcal{R}_{1}}(2 \sqrt{x}, \sqrt{2 y}, \sqrt{2 z}) K_{B}[\varphi](2 \sqrt{x}, \sqrt{2 y}, \sqrt{2 z}) \mathrm{d} F(x) \mathrm{d} F(y) \mathrm{d} F(z) \\
& =\frac{(4 \pi \sqrt{2})^{3}}{4 \pi} \int_{\mathbb{R}_{\geq 0}^{3},(y-x)(z-x) \neq 0, x>0, y>0, z>0, y+z>x} \frac{\Delta \varphi(x, y, z)}{\sqrt{x y z}} \\
& \times\left\{\int_{|\sqrt{x}-\sqrt{y}| \vee\left|\sqrt{x_{*}}-\sqrt{z}\right|}^{(\sqrt{x}+\sqrt{y}) \wedge\left(\sqrt{x_{*}}+\sqrt{z}\right)} \mathrm{d} s \int_{0}^{2 \pi} \frac{s \sqrt{z}}{s \sqrt{z}+Y_{*} \sqrt{y}} \Phi\left(\sqrt{2} s, \sqrt{2} Y_{*}\right) \mathrm{d} \theta\right. \\
& \left.+\int_{|\sqrt{x}-\sqrt{z}| \vee\left|\sqrt{x_{*}}-\sqrt{y}\right|}^{(\sqrt{x}+\sqrt{z}) \wedge\left(\sqrt{x_{*}}+\sqrt{y}\right)} \mathrm{d} s \int_{0}^{2 \pi} \frac{s \sqrt{y}}{s \sqrt{y}+\widetilde{Y}_{* \sqrt{z}}} \Phi\left(\sqrt{2} s, \sqrt{2} \widetilde{Y}_{*}\right) \mathrm{d} \theta\right\} \mathrm{d} F(x) \mathrm{d} F(y) \mathrm{d} F(z) \\
& =4 \pi \sqrt{2} \int_{\mathbb{R}_{\geq 0}^{3},(y-x)(z-x) \neq 0, x>0, y>0, z>0, y+z>x} \frac{\Delta \varphi(x, y, z)}{4 \pi \sqrt{x y z}} \\
& \times \int_{|\sqrt{x}-\sqrt{y}| \vee\left|\sqrt{x_{*}}-\sqrt{z}\right|}^{(\sqrt{x}+\sqrt{y}) \wedge\left(\sqrt{x_{*}}+\sqrt{z}\right)} \mathrm{d} s \int_{0}^{2 \pi} \Phi\left(\sqrt{2} s, \sqrt{2} Y_{*}\right) \mathrm{d} \theta \mathrm{d} F(x) \mathrm{d} F(y) \mathrm{d} F(z) \\
& =4 \pi \sqrt{2} \int_{\mathbb{R}_{\geq 0}^{3}, x>0, y>0, z>0} \mathcal{K}[\varphi](x, y, z) \mathrm{d} F(x) \mathrm{d} F(y) \mathrm{d} F(z)
\end{aligned}
$$

where here and below we used the fact that $(y-x)(z-x)=0$ or $y+z \leq x \Longrightarrow \mathcal{K}[\varphi](x, y, z)=0$.

To compute $I_{2}$ we recall definition of $X, Y$ in $(6.14),(6.15)$ to see that $\left(r, r^{\prime}, r_{*}^{\prime}\right) \in \mathcal{R}_{2} \Longrightarrow$ $r=0, X=r^{\prime}>0, Y=r_{*}^{\prime}>0$, and so letting $x=0, y=r^{\prime 2} / 2>0, z=r_{*}^{\prime 2} / 2>0$ gives

$$
\begin{aligned}
& K_{B}[\varphi]\left(0, r^{\prime}, r_{*}^{\prime}\right)=\frac{1}{(4 \pi)^{3}} \int_{-1}^{1} \mathrm{~d} t \int_{0}^{2 \pi} 1_{\left\{r_{*}^{\prime}>0\right\}} \frac{\Delta \varphi\left(0, r^{\prime 2} / 2, r_{*}^{\prime 2} / 2\right)}{r^{\prime} r_{*}^{\prime}} \Phi\left(r^{\prime}, r_{*}^{\prime}\right) \mathrm{d} \theta \\
& =\frac{2 \pi}{(4 \pi)^{3}} W(0, y, z) \Delta \varphi(0, y, z)=\frac{2 \pi}{(4 \pi)^{3}} \mathcal{K}[\varphi](0, y, z)
\end{aligned}
$$

and so

$$
\begin{aligned}
I_{2} & =(4 \pi \sqrt{2})^{3} \int_{\mathbb{R}_{\geq 0}^{3}} \mathbf{1}_{\mathcal{R}_{2}}(\sqrt{2 x}, \sqrt{2 y}, \sqrt{2 z}) \mathcal{K}[\varphi](\sqrt{2 x}, \sqrt{2 y}, \sqrt{2 z}) \mathrm{d} F(x) \mathrm{d} F(y) \mathrm{d} F(z) \\
& =4 \pi \sqrt{2} \int_{\mathbb{R}_{\geq 0}^{3}, x=0, y>0, z>0} \mathcal{K}(x, y, z) \mathrm{d} F(x) \mathrm{d} F(y) \mathrm{d} F(z) .
\end{aligned}
$$

For the integral $I_{3}$, we have $\left(r, r^{\prime}, r_{*}^{\prime}\right) \in \mathcal{R}_{3} \Longrightarrow r^{\prime}=0, r_{*}^{\prime}>X=r>0, Y=\sqrt{r_{*}^{\prime 2}-r^{2}}$ and so letting $x=r^{2} / 2>0, y=0, z=r_{*}^{\prime 2} / 2>0$ gives

$$
\begin{aligned}
& K_{B}[\varphi]\left(r, 0, r_{*}^{\prime}\right)=\frac{2}{(4 \pi)^{3}} \int_{-1}^{1} \mathrm{~d} t \int_{0}^{2 \pi} 1_{\left\{r_{*}^{\prime}>r\right\}} \frac{\Delta \varphi\left(r^{2} / 2,0, r_{*}^{\prime 2} / 2\right)}{r r_{*}^{\prime}} \Phi\left(r, \sqrt{{r_{*}^{\prime}}^{2}-r^{2}}\right) \mathrm{d} \theta \\
& =\frac{2}{(4 \pi)^{3}} 4 \pi 1_{\{z>x\}} \frac{\Delta \varphi(x, 0, z)}{2 \sqrt{x z}} \Phi(\sqrt{2 x}, \sqrt{2(z-x)})=\frac{2}{(4 \pi)^{2}} 1_{\{z>x>0\}} \mathcal{K}[\varphi](x, 0, z) .
\end{aligned}
$$


Then using the symmetry $\mathcal{K}[\varphi](x, y, z) \equiv \mathcal{K}[\varphi](x, z, y)$ we have

$$
\begin{aligned}
I_{3} & =(4 \pi \sqrt{2})^{3} \int_{\mathbb{R}_{\geq 0}^{3}} \mathbf{1}_{\mathcal{R}_{3}}(\sqrt{2 x}, \sqrt{2 y}, \sqrt{2 z}) \mathcal{K}[\varphi](\sqrt{2 x}, \sqrt{2 y}, \sqrt{2 z}) \mathrm{d} F(x) \mathrm{d} F(y) \mathrm{d} F(z) \\
& =4 \pi \sqrt{2} \cdot 2 \int_{\mathbb{R}_{\geq 0}^{3}, y=0, z>x>0} \mathcal{K}[\varphi](x, y, z) \mathrm{d} F(x) \mathrm{d} F(y) \mathrm{d} F(z) \\
& =4 \pi \sqrt{2} \int_{\mathbb{R}_{\geq 0}^{3}, y=0, z>x>0} \mathcal{K}[\varphi](x, y, z) \mathrm{d} F(x) \mathrm{d} F(y) \mathrm{d} F(z) \\
& +4 \pi \sqrt{2} \int_{\mathbb{R}_{\geq 0}^{3}, z=0, y>x>0} \mathcal{K}[\varphi](x, y, z) \mathrm{d} F(x) \mathrm{d} F(y) \mathrm{d} F(z) .
\end{aligned}
$$

Taking sum $I_{1}+I_{2}+I_{3}$ and using (6.21) we obtain (6.19).

\subsection{Existence and positivity of some potential $U(|\mathbf{x}|)$.}

Lemma 6.9. Let $V \in C_{b}\left(\mathbb{R}_{\geq 0}\right) \cap C^{2}\left(\mathbb{R}_{>0}\right), V_{1}(r)=V(r) r$, and suppose that $\frac{\mathrm{d}^{2}}{\mathrm{~d} r^{2}} V_{1}(r)$ is nondecreasing in $(0, \infty)$ and there are constants $0<\delta<1, C>0$ such that

$$
\left|\frac{\mathrm{d}}{\mathrm{d} r} V_{1}(r)\right| \leq \frac{C}{1+r^{2 \delta}}, \quad r>0 ; \quad \int_{0}^{\infty}\left(1+r^{\delta}\right)\left|\frac{\mathrm{d}^{2}}{\mathrm{~d} r^{2}} V_{1}(r)\right| \mathrm{d} r<\infty .
$$

Let

$$
U(\rho)=\frac{1}{2 \pi^{2} \rho^{3}} \int_{0}^{\infty}\left(-\frac{\mathrm{d}^{2}}{\mathrm{~d} r^{2}} V_{1}(r)\right) \sin (\rho r) \mathrm{d} r, \quad \rho>0
$$

Then $U(\rho) \geq 0$ for all $\rho \in(0, \infty)$ and $\widehat{U}(\xi)=V(|\xi|)$ for all $\xi \in \mathbb{R}^{3}$, where $\widehat{U}$ is the Fourier transform of the function $\mathbf{x} \mapsto U(|\mathbf{x}|)$ in terms of theory of generalized functions.

Proof. To prove the positivity of $U$ we will use the following property: if $f \in L^{1}((0, \infty))$ is non-negative and non-increasing in $(0, \infty)$, then

$$
\int_{0}^{\infty} f(r) \sin (\rho r) \mathrm{d} r \geq 0 \quad \forall \rho>0 .
$$

The proof of (6.22) is easy: first for any $0<a<b<\infty$, according to the second mean-value theorem of integration, there is $c \in(a, b)$ such that $\int_{a}^{b} f(r) \sin (\rho r) \mathrm{d} r=f(a) \int_{a}^{c} \sin (\rho r) \mathrm{d} r$, then use the fact that $0 \leq f \in L^{1}((0, \infty))$ implies $\liminf _{a \rightarrow 0+} f(a) a^{2}=0$. These deduce (6.22).

To prove the lemma we use approximation: for any $\varepsilon>0$, let

$$
U_{\varepsilon}(\mathbf{x})=(2 \pi)^{-3} \int_{\mathbb{R}^{3}} e^{-\varepsilon|\xi|} V(|\xi|) e^{\mathrm{ix} \cdot \xi} \mathrm{d} \xi, \quad \mathbf{x} \in \mathbb{R}^{3}
$$

$U_{\varepsilon}(\mathbf{x})$ is obviously radially symmetric, so if we denote $U_{\varepsilon}(\rho)=\left.U_{\varepsilon}(\mathbf{x})\right|_{|\mathbf{x}|=\rho}$, then

$$
U_{\varepsilon}(\rho)=\frac{1}{2 \pi^{2} \rho} \int_{0}^{\infty} e^{-\varepsilon r} V_{1}(r) \sin (\rho r) \mathrm{d} r, \quad \rho>0 .
$$


Next using integration by parts twice we deduce

$$
\begin{aligned}
& \int_{0}^{\infty} e^{-\varepsilon r} V_{1}(r) \sin (\rho r) \mathrm{d} r=\frac{1}{\varepsilon} \int_{0}^{\infty} e^{-\varepsilon r} \frac{\mathrm{d}}{\mathrm{d} r} V_{1}(r) \sin (\rho r) \mathrm{d} r \\
& +\frac{1}{\varepsilon^{2}} \int_{0}^{\infty} e^{-\varepsilon r} \frac{\mathrm{d}}{\mathrm{d} r} V_{1}(r) \cos (\rho r) \rho \mathrm{d} r-\frac{\rho^{2}}{\varepsilon^{2}} \int_{0}^{\infty} e^{-\varepsilon r} V_{1}(r) \sin (\rho r) \mathrm{d} r
\end{aligned}
$$

For the integral that contains $\cos (\rho r)$, integrating by parts again and reorganizing the results we obtain

$$
\begin{aligned}
U_{\varepsilon}(\rho) & =\frac{1}{2 \pi^{2} \rho\left(\varepsilon^{2}+\rho^{2}\right)}\left(2 \varepsilon \int_{0}^{\infty} e^{-\varepsilon r} \frac{\mathrm{d}}{\mathrm{d} r} V_{1}(r) \sin (\rho r) \mathrm{d} r+\int_{0}^{\infty} e^{-\varepsilon r}\left(-\frac{\mathrm{d}^{2}}{\mathrm{~d} r^{2}} V_{1}(r)\right) \sin (\rho r) \mathrm{d} r\right) \\
& =U_{1, \varepsilon}(\rho)+U_{2, \varepsilon}(\rho), \quad \rho>0 .
\end{aligned}
$$

From the assumption we see that $-\frac{\mathrm{d}^{2}}{\mathrm{~d} r^{2}} V_{1}(r)$ is non-increasing in $(0, \infty)$ and so by integrability we have $-\frac{\mathrm{d}^{2}}{\mathrm{~d} r^{2}} V_{1}(r) \geq \lim _{R \rightarrow \infty}\left(-\frac{\mathrm{d}^{2}}{\mathrm{~d} r^{2}} V_{1}(R)\right)=0$ for all $r>0$. From this we see that $\frac{\mathrm{d}}{\mathrm{d} r} V_{1}(r)$ is non-increasing in $(0, \infty)$ and so $\frac{\mathrm{d}}{\mathrm{d} r} V_{1}(r) \geq \lim _{R \rightarrow \infty} \frac{\mathrm{d}}{\mathrm{d} r} V_{1}(R)=0$ for all $r>0$. Thus the functions $-\frac{\mathrm{d}^{2}}{\mathrm{~d} r^{2}} V_{1}(r), e^{-\varepsilon r} \frac{\mathrm{d}}{\mathrm{d} r} V_{1}(r), e^{-\varepsilon r}\left(-\frac{\mathrm{d}^{2}}{\mathrm{~d} r^{2}} V_{1}(r)\right)$ are non-negative and non-increasing in $(0, \infty)$ and belong to $L^{1}((0, \infty))$. By (6.22) we conclude $U(\rho) \geq 0, U_{1, \varepsilon}(\rho) \geq 0, U_{2, \varepsilon}(\rho) \geq 0$ hence $U_{\varepsilon}(\rho) \geq 0$ for all $\rho>0(\forall \varepsilon>0)$. Next we show that

$$
\sup _{0<\varepsilon \leq 1} U_{\varepsilon}(\rho) \leq C_{3} \frac{1}{\rho^{3-\delta}}, \quad \lim _{\varepsilon \rightarrow 0} U_{\varepsilon}(\rho)=U(\rho) \quad \forall \rho>0
$$

Here and below the constants $C_{i}>0(i=1,2,3,4)$ depend only on $V, C$ and $\delta$.

In fact for all $\varepsilon>0, \rho>0$ we have

$$
0 \leq U_{1, \varepsilon}(\rho) \leq \frac{C}{\pi^{2} \rho^{3}} \varepsilon \int_{0}^{\infty} e^{-\varepsilon r} \frac{(\rho r)^{\delta}}{r^{2 \delta}} \mathrm{d} r=\frac{C_{1}}{\rho^{3-\delta}} \varepsilon^{\delta}
$$

and (using $|\sin (x)| \leq x^{\delta}$ for all $x \geq 0$ )

$$
\left.\left.\frac{1}{2 \pi^{2} \rho\left(\varepsilon^{2}+\rho^{2}\right)} e^{-\varepsilon r} \mid \frac{\mathrm{d}^{2}}{\mathrm{~d} r^{2}} V_{1}(r)\right)|| \sin (\rho r)\left|\leq \frac{C_{2}}{\rho^{3-\delta}} r^{\delta}\right| \frac{\mathrm{d}^{2}}{\mathrm{~d} r^{2}} V_{1}(r)\right) \mid, \quad r>0 .
$$

This gives the first inequality in (6.23). From (6.25) and Lebesgue dominated convergence we obtain

$$
\lim _{\varepsilon \rightarrow 0+} U_{2, \varepsilon}(\rho)=\lim _{\varepsilon \rightarrow 0+} \frac{1}{2 \pi^{2} \rho\left(\varepsilon^{2}+\rho^{2}\right)} \int_{0}^{\infty} e^{-\varepsilon r}\left(-\frac{\mathrm{d}^{2}}{\mathrm{~d} r^{2}} V_{1}(r)\right) \sin (\rho r) \mathrm{d} r=U(\rho)
$$

for all $\rho>0$. This together with (6.24) gives (6.23).

Now let $\mathcal{S}\left(\mathbb{R}^{3}\right)$ be the class of Schwartz functions on $\mathbb{R}^{3}$. For any $\psi \in \mathcal{S}\left(\mathbb{R}^{3}\right)$ we have $\widehat{\psi} \in \mathcal{S}\left(\mathbb{R}^{3}\right)$ and it holds the inverse formula

$$
\psi(\xi)=2 \pi)^{-3} \int_{\mathbb{R}^{3}} \widehat{\psi}(\mathbf{x}) e^{\mathrm{i} \mathbf{x} \cdot \xi} \mathrm{d} \mathbf{x} \quad \forall \xi \in \mathbb{R}^{3}
$$


from which and Fubini theorem we obtain

$$
\int_{\mathbb{R}^{3}} U_{\varepsilon}(|\mathbf{x}|) \widehat{\psi}(\mathbf{x}) \mathrm{d} \mathbf{x}=\int_{\mathbb{R}^{3}} e^{-\varepsilon|\xi|} V(|\xi|) \psi(\xi) \mathrm{d} \xi, \quad \varepsilon>0 .
$$

Since

$$
\begin{gathered}
\sup _{0<\varepsilon \leq 1}\left|U_{\varepsilon}(|\mathbf{x}|) \widehat{\psi}(\mathbf{x})\right| \leq C_{3}|\widehat{\psi}(\mathbf{x})| \frac{1}{|\mathbf{x}|^{3-\delta}}, \quad \sup _{\varepsilon>0}\left|e^{-\varepsilon|\xi|} V(|\xi|) \psi(\xi)\right| \leq C_{4}|\psi(\xi)|, \\
\lim _{\varepsilon \rightarrow 0+} U_{\varepsilon}(|\mathbf{x}|) \widehat{\psi}(\mathbf{x})=U(|\mathbf{x}|) \widehat{\psi}(\mathbf{x}), \quad \lim _{\varepsilon \rightarrow 0+} e^{-\varepsilon|\xi|} V(|\xi|) \psi(\xi)=V(|\xi|) \psi(\xi)
\end{gathered}
$$

for all $\mathbf{x} \in \mathbb{R}^{3} \backslash\{0\}, \xi \in \mathbb{R}^{3}$, it follows from (6.26) and Lebesgue dominated convergence that

$$
\int_{\mathbb{R}^{3}} U(|\mathbf{x}|) \widehat{\psi}(\mathbf{x}) \mathrm{d} \mathbf{x}=\int_{\mathbb{R}^{3}} V(|\xi|) \psi(\xi) \mathrm{d} \xi \quad \forall \psi \in \mathcal{S}\left(\mathbb{R}^{3}\right) .
$$

Thus according to the definition of the Fourier transform of generalized functions we conclude $\widehat{U}(\xi)=V(|\xi|)$.

If we choose $V(r)=\frac{1}{1+r^{\eta}}, 0<\eta<1$, then it is easily checked that $V$ satisfies the conditions in Lemma 6.9 with $\delta=\eta / 2$.

Acknowledgments. We are grateful to a referee for helpful suggestions on the presentation of the paper. This work was supported by National Natural Science Foundation of China Grant No.11771236.

Note: This is just an optimal version of the last version which has been published in Journal of Statistical Physics, 175(2019), no. 2, 289-350. In the present version, the collision kernel $B\left(\mathbf{v}-\mathbf{v}_{*}, \omega\right)$ is slightly extended to a common one satisfying (1.4),(1.5), and the constant $\eta$ in Assumption 1.1 is only assumed $\eta \geq 0$. The proofs for the corresponding results, e.g. Proposition 3.4, Proposition 4.2, Theorem 4.4, are also updated easily.

\section{References}

[1] Arkeryd, L.: On low temperature kinetic theory; spin diffusion, Bose Einstein condensates, anyons. J. Stat. Phys. 150, 1063-1079 (2013)

[2] Arkeryd, L., and Nouri, A.: Bose condensates in interaction with excitations: a kinetic model. Comm. Math. Phys. 310, 765-788 (2012)

[3] Bandyopadhyay, J., Velázquez, J.J.L.: Blow-up rate estimates for the solutions of the bosonic Boltzmann-Nordheim equation. J. Math. Phys. 56 (2015), 063302, 27 pp. doi: $10.1063 / 1.4921917$.

[4] Benedetto, D., Pulvirenti, M., Castella, F., Esposito, R.: On the weak-coupling limit for bosons and fermions. Math. Models Methods Appl. Sci. 15, 1811-1843 (2005) 
[5] Briant, M., Einav, A.: On the Cauchy problem for the homogeneous Boltzmann-Nordheim equation for bosons: local existence, uniqueness and creation of moments. J. Stat. Phys. 163, 1108-1156 (2016)

[6] Carlen, E.A., Carvalho, M.C., Lu, X.: On strong convergence to equilibrium for the Boltzmann equation with soft potentials. J. Stat. Phys. 135, 681-736 (2009)

[7] Chapman, S., Cowling, T.G.: The Mathematical Theory of Non-Uniform Gases. Third Edition (Cambridge University Press, 1970).

[8] Demengel, F., Temam, R. Convex functions of a measure and applications. Indiana Univ. Math. J. 33, no. 5, 673-709 (1984).

[9] Erdös, L., Salmhofer, M., Yau, H.-T.: On the quantum Boltzmann equation. J. Stat. Phys. 116, 367-380 (2004).

[10] Escobedo, M., Mischler, S.: On a quantum Boltzmann equation for a gas of photons. J. Math. Pures Appl. (9) 80, no. 5, 471-515 (2001).

[11] Escobedo, M., Mischler, S., Valle, M.A.: Homogeneous Boltzmann equation in quantum relativistic kinetic theory. Electronic Journal of Differential Equations, Monograph, 4. Southwest Texas State University, San Marcos, TX, 2003. 85 pp.

[12] Escobedo, M., Mischler, S., Velázquez, J.J.L.: Singular solutions for the Uehling-Uhlenbeck equation. Proc. Roy. Soc. Edinburgh 138A, 67-107 (2008)

[13] Escobedo, M., Velázquez, J.J.L.: On the blow up and condensation of supercritical solutions of the Nordheim equation for bosons. Comm. Math. Phys. 330, 331-365 (2014)

[14] Escobedo, M., Velázquez, J.J.L.: Finite time blow-up and condensation for the bosonic Nordheim equation. Invent. Math. 200, 761-847 (2015)

[15] Escobedo, M., Tran, M.-B.: Convergence to equilibrium of a linearized quantum Boltzmann equation for bosons at very low temperature. Kinet. Relat. Models 8, 493-531 (2015)

[16] Josserand, C., Pomeau, Y., Rica, S.: Self-similar singularities in the kinetics of condensation. J. Low Temp. Phys. 145, 231-265 (2006)

[17] Li, W., Lu, X.: Global existence of solutions of the Boltzmann equation for Bose-Einstein particles with anisotropic initial data. J. Funct. Anal. 276, 231-283 (2019)

[18] Lu, X.: A modified Boltzmann equation for Bose-Einstein particles: isotropic solutions and long-time behavior. J. Statist. Phys. 98, 1335-1394 (2000) 
[19] Lu, X.: On isotropic distributional solutions to the Boltzmann equation for Bose-Einstein particles. J. Statist. Phys. 116, 1597-1649 (2004)

[20] Lu, X.: The Boltzmann equation for Bose-Einstein particles: velocity concentration and convergence to equilibrium. J. Statist. Phys. 119, 1027-1067 (2005)

[21] Lu, X.: The Boltzmann equation for Bose-Einstein particles: condensation in finite time. J. Stat. Phys. 150, 1138-1176 (2013)

[22] Lu, X.: Long time convergence of the Bose-Einstein condensation. J. Stat. Phys. 162, 652-670 (2016)

[23] Lu, X.: Long time strong convergence to Bose-Einstein distribution for low temperature. Kinet. Relat. Models 11, 715-734 (2018)

[24] Lukkarinen, J., Spohn, H.: Not to normal order-notes on the kinetic limit for weakly interacting quantum fluids. J. Stat. Phys. 134, 1133-1172 (2009)

[25] Markowich, P.A., Pareschi, L.: Fast conservative and entropic numerical methods for the boson Boltzmann equation. Numer. Math. 99, 509-532 (2005)

[26] Nordheim, L.W.: On the kinetic methods in the new statistics and its applications in the electron theory of conductivity. Proc. Roy. Soc. London Ser. A 119, 689-698 (1928)

[27] Nouri, A: Bose-Einstein condensates at very low temperatures: a mathematical result in the isotropic case. Bull. Inst. Math. Acad. Sin. (N.S.) 2, 649-666 (2007)

[28] Rudin, W: Real and Complex Analysis. Third edition. McGraw-Hill Book Co., New York, 1987. xiv+416 pp. ISBN: 0-07-054234-1 00A05 (26-01 30-01 46-01)

[29] Semikov, D.V., Tkachev, I.I.: Kinetics of Bose condensation. Phys. Rev. Lett. 74, 30933097 (1995)

[30] Semikov, D.V., Tkachev, I.I.: Condensation of Bose in the kinetic regime. Phys. Rev. D 55, 489-502 (1997)

[31] Spohn, H.: Kinetics of the Bose-Einstein condensation. Physica D 239, 627-634 (2010)

[32] Uehling, E.A., Uhlenbeck, G.E.: Transport phenomena in Einstein-Bose and Fermi-Dirac gases, I, Phys. Rev. 43, 552-561 (1933)

[33] Villani, C.: A review of mathematical topics in collisional kinetic theory. Handbook of mathematical fluid dynamics, Vol.I, 71-305, North-Holland, Amsterdam, 2002. 
[34] Villani, C.: Cercignani's conjecture is sometimes true and always almost true. Comm. Math. Phys. 234, 455-490 (2003) 